\title{
A compositional letter code in high-level visual cortex explains how we read jumbled words
}

\author{
Aakash Agrawal' ${ }^{1}$, K.V.S. Hari² \& S. P. Arun $^{3^{*}}$ \\ ${ }^{1}$ Centre for BioSystems Science \& Engineering, ${ }^{2}$ Department of Electrical \\ Communication Engineering \& ${ }^{3}$ Centre for Neuroscience \\ Indian Institute of Science, Bangalore, 560012, India
}

${ }^{\star}$ Correspondence to: S. P. Arun (sparun@iisc.ac.in) 
ABSTRACT

We read words and even jubmled wrods effortlessly, but the neural

3 representations underlying this remarkable ability remain unknown. We hypothesized

4 that word processing is driven by a visual representation that is compositional i.e. with

5 string responses systematically related to letters. To test this hypothesis, we devised

6 a model in which neurons tuned to letter shape respond to longer strings by linearly

7 summing letter responses. This letter model explained human performance in both

8 visual search as well as word reading tasks. Brain imaging revealed that viewing a

9 string activates this compositional letter code in the lateral occipital (LO) region, and

10 that subsequent comparisons to known words are computed by the visual word form

11 area (VWFA). Thus, seeing a word activates a compositional letter code that enables

12 efficient reading. 


\section{INTRODUCTION}

Reading is a recent cultural invention, yet we are remarkably efficient at reading

words and even jmulbed wrods (Figure 1A). What makes a jumbled word easy or hard

to read? This question has captured the popular imagination through demonstrations such as the Cambridge University effect (Rawlinson, 1976; Grainger and Whitney, 2004), depicted in Figure 1A. Reading a word or a jumbled word can be influenced by a variety of factors (Norris, 2013; Grainger, 2018). Word reading is easy when similar shapes are substituted (Perea et al., 2008; Perea and Panadero, 2014), when the first and last letters are preserved (Rayner et al., 2006), when there are fewer transpositions (Gomez et al., 2008) and when word shape is preserved (Norris, 2013; Grainger, 2018). Word reading is also easier for words with frequent bigrams or trigrams, for frequent words and for shuffled words that preserve intermediate units such as consonant clusters or morphemes (Norris, 2013; Grainger, 2018). Despite these insights, it is not clear how these factors combine, what their distinct contributions are, and more generally, how word representations relate to letter representations.

Here, we hypothesized that word reading is enabled by a purely visual representation. To probe purely visual processing, we devised a visual search task in which subjects had to find an oddball target among distractors. This task does not require any explicit reading and is driven by shape representations in visual cortex (Sripati and Olson, 2010a; Zhivago and Arun, 2014). An example visual search array containing two oddball targets is shown in Figure 1B. It can be seen that finding OFRGET is easy among FORGET whereas finding FOGRET is hard (Figure 1B). This difference in visual similarity (Figure 1C) explains why transposing the middle letters renders a word easier to read than transposing its edge letters. This example suggests 
that word reading could be explained by purely visual processing as indexed by visual search. However, subjects may have been reading during visual search, thereby activating non-visual lexical or linguistic factors.

To overcome this confound, we asked whether visual search involving letter strings can be explained using a neurally plausible model containing only visual factors. We drew upon two well-established principles of object representations in high-level visual cortex. First, images that are perceptually similar elicit similar activity in single neurons (Op de Beeck et al., 2001; Sripati and Olson, 2010a; Zhivago and Arun, 2014). Accordingly we used visual search for single letters to create artificial neurons tuned for single letters. Second, the neural response to multiple objects is an average of the response to the individual objects, a phenomenon known as divisive normalization (Zoccolan et al., 2005; Ghose and Maunsell, 2008; Zhivago and Arun, 2014). Accordingly, we created neural responses to letter strings as a linear sum of single letter responses. In contrast to an influential proposal that requires neurons tuned to letter combinations (Dehaene et al., 2005, 2010), our model only assumes neurons tuned for letter shape and retinal position, as observed in high-level visual cortex (Lehky and Tanaka, 2016). It does not capture any information about specialized detectors for longer strings, or about other lexical or linguistic factors. We used this model to explain human performance on visual search as well as word recognition tasks. Finally, using brain imaging, we identified the neural substrates for both the letter code as well as subsequent lexical decisions. 
A

AOCCDRNIG TO A RSEEARCH AT CMABRIGDE UINERVTISY, IT DEOSN'T MTTAER IN WAHT OREDR THE LTTEERS IN A WROD ARE, THE OLNY IPRMOETNT TIHNG IS TAHT THE FRIST AND LSAT LTTEER BE AT THE RGHIT PCLAE. THE RSET CAN BE A TOATL MSES AND YOU CAN SITLL RAED IT WOUTHIT A PORBELM. TIHS IS BCUSEAE THE HUAMN MNID DEOS NOT RAED ERVEY LTETER BY ISTLEF, BUT THE WROD AS A WLOHE.

C

Example Search array

\begin{tabular}{|ccc|}
\hline & FORGET & FORGET \\
FORGET & & \\
& OFRGET & FORGET \\
FORGET & FORGET \\
FORGET & FORGET & FORGET \\
FORGET & & \\
FORGET & FORGET & FOGRET \\
& FORGET & FORGET \\
\hline
\end{tabular}

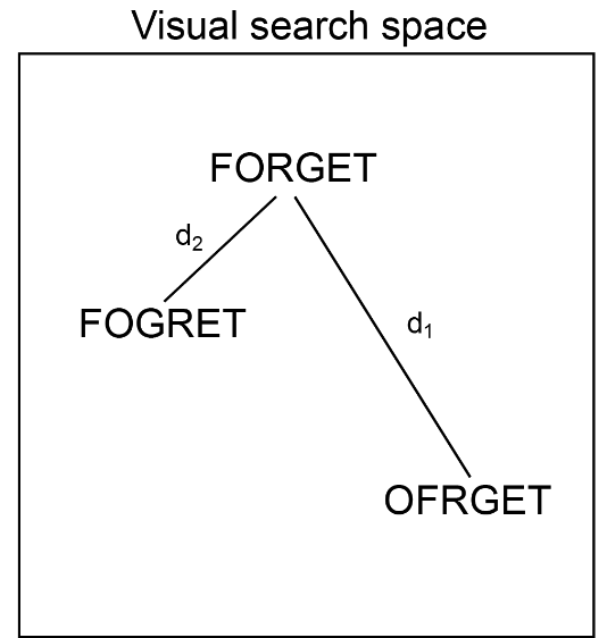

\section{Figure 1. Reading jumbled words}

(A) We are extremely good at reading jumbled words, as illustrated by the popular Cambridge University effect.

(B) Visual search array showing two oddball targets (OFRGET \& FOGRET) among many instances of FORGET. OFRGET is easy to find but not FOGRET.

(C) Schematic representation of these strings in visual search space, arranged such that similar items (corresponding to harder searches) are nearby. Thus, FOGRET is closer to FORGET compared to OFRGET (i.e. $d_{1}>d_{2}$ ). 


\section{RESULTS}

We performed five key experiments. In Experiment 1, subjects performed visual search involving single letters, and we used this to construct artificial neurons tuned for letter shape. In Experiments 2-4, we show that search for longer strings can be predicted using these artificial neurons with a simple compositional rule. In Experiment 5, we show that this model also explains human performance on a commonly studied word recognition task. Finally, in Experiment 6, we measured brain activations during word recognition to elucidate the underlying neural representations.

\section{Experiment 1: Single letter searches}

We recruited 16 subjects to perform an oddball visual search task involving uppercase letters $(n=26)$, lowercase letters $(n=26)$ and digits $(n=10)$. An example search is shown in Figure 2A. Subjects were highly consistent in their responses (splithalf correlation between average search times of odd- and even-numbered subjects: $r=0.87, p<0.00005)$. We calculated the reciprocal of search times for each letter pair which is a measure of distance between them (Arun, 2012). These letter dissimilarities were significantly correlated with previously reported subjective dissimilarity ratings (Section S1).

Since shape dissimilarity in visual search matches closely with neural

91 dissimilarity in visual cortex (Sripati and Olson, 2010a; Zhivago and Arun, 2014), we asked whether these letter distances can be used to reconstruct the underlying neural responses to single letters. To do so, we performed a multidimensional scaling (MDS) analysis, which finds the n-dimensional coordinates of all letters such that their distances match the observed visual search distances. In the resulting plot for 2 dimensions for uppercase letters (Figure 2B), nearby letters correspond to small 
97 distances i.e. long search times. The coordinates of letters along a particular

98 dimension can then be taken as the putative response of a single neuron. For example,

99 the first dimension represents the activity of a neuron that responds strongest to the

100 letter $\mathrm{O}$ and weakest to $\mathrm{X}$ (Figure $2 \mathrm{C}$ ). Likewise the second dimension corresponds to

101 a neuron that responds strongest to $L$ and weakest to $E$ (Figure $2 C$ ). We note that the

102 same set of distances can be obtained from a different set of neural responses: a

103 simple coordinate axis rotation would result in another set of neural responses with an

104 equivalent match to the observed distances. Thus, the estimated activity from MDS

105 represents one possible solution to how neurons should respond to individual letters

106 so as to collectively produce behaviour.

107 As expected, increasing the number of MDS dimensions led to increased match

108 to the observed letter dissimilarities (Figure 2D). Taking 10 MDS dimensions, which

109 explain nearly $95 \%$ of the variance, we obtained the single letter responses of 10 such

110 artificial neurons. We used these single letter responses to predict their response to

111 longer letter strings in all the experiments. Varying this choice yielded qualitatively

112 similar results. Analogous results for all letters and numbers are shown in Section S1. 
A

$\begin{array}{cccc}N & N & N & N \\ N & N & W & N \\ N & N & N & N \\ N & N & N & N \\ N & T & N & N \\ N & N & N & N \\ N & N & N & N\end{array}$

114
B

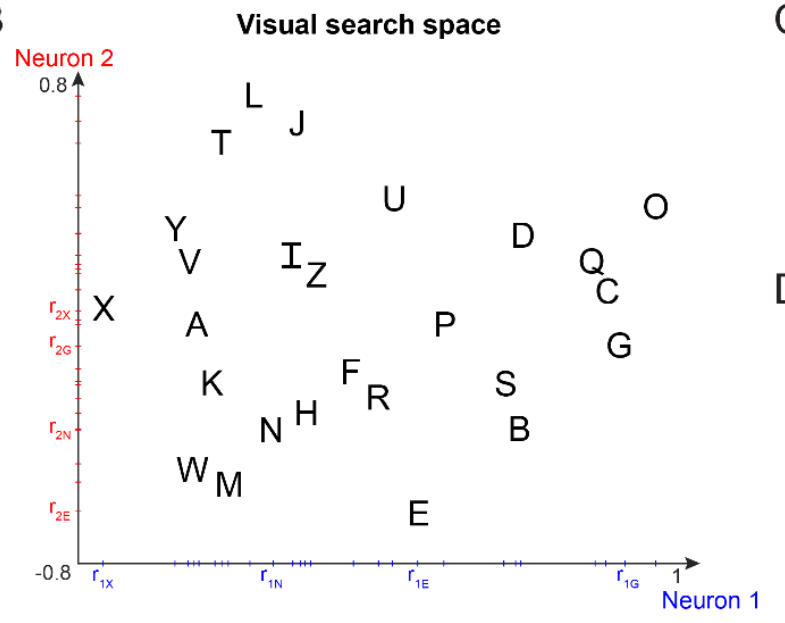

C
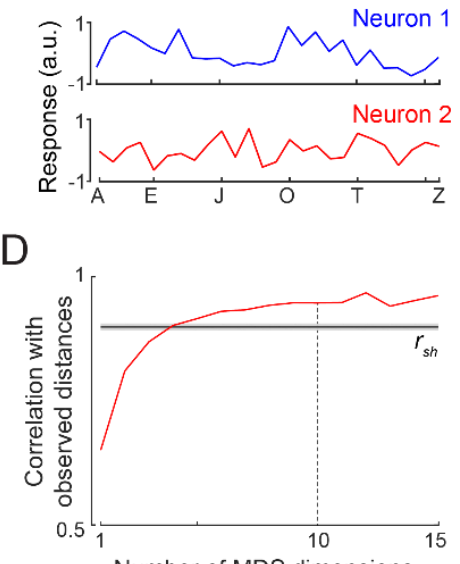

Figure 2. Single letter discrimination (Experiment 1)

(A) Visual search array showing two oddball targets (W \& T) among many Ns. It can be seen that finding $\mathrm{W}$ is harder compared to finding $\mathrm{T}$. The actual experiment comprised search arrays with only one oddball target among 15 distractors.

(B) Visual search space for uppercase letters obtained by multidimensional scaling of observed dissimilarities. Nearby letters represent hard searches. Distances in this $2 \mathrm{D}$ plot are highly correlated with the observed distances $(r=0.82, p<0.00005)$. Letter activations along the $\mathrm{x}$-axis are taken as responses of Neuron 1 (blue), and along the $y$-axis are taken as Neuron 2 (red), etc. The tick marks indicate the response of each letter along that neuron.

(C) Responses of Neuron 1 and Neuron 2 shown separately for each letter. Neuron 1 responds best to $O$, whereas Neuron 2 responds best to $L$.

(D) Correlation between observed distances and MDS embedding as a function of number of MDS dimensions. The black line represents the split-half correlation with error bars representing s.d calculated across 100 random splits. 


\section{Experiment 2: Bigram searches}

Next we proceeded to ask whether searches for longer strings can be explained

using single letter responses. In Experiment 2, we asked subjects to perform oddball searches involving bigrams. An example search is depicted in Figure 3A. It can be seen that, finding TA among AT is harder than finding UT among AT. Thus, letter

136 transpositions are more similar compared to letter substitutions, consistent with the

137 classic results on reading (Norris, 2013; Grainger, 2018). To characterize the effect of bigram frequency, we included both frequent bigrams (e.g. IN, TH) and infrequent bigrams (e.g. $\mathrm{MH}, \mathrm{HH}$ ). As before, subjects were highly consistent in their performance

140 (split-half correlation between odd and even numbered subjects across all bigrams: $r$

$141=0.82, \mathrm{p}<0.00005)$.

Next we asked whether bigram search performance can be explained using neurons tuned to single letters estimated from Experiment 1. The essential principle

144 for constructing bigram responses is depicted in Figure 3B. In monkey visual cortex,

145 the response of single neurons to two simultaneously presented objects is an average 146 of the single object responses (Zoccolan et al., 2005; Zhivago and Arun, 2014; Pramod and Arun, 2018). This averaging can easily be biased through changes in divisive normalization (Ghose and Maunsell, 2008). Therefore we took the response of each neuron to a bigram to be a weighted sum of its responses to the constituent letters

150 (Figure 3B). Specifically, the response of a neuron to the bigram $A B$ is given by $r_{A B}=$ $151 w_{1} r_{A}+w_{2} r_{B}$, where $r_{A B}$ is the response to $A B, r_{A}$ and $r_{B}$ are its responses to the constituent letters $A \& B$, and $w_{1}, w_{2}$ are the summation weights reflecting the importance of letters $A \& B$ in the summation. Note that if $w_{1}=w_{2}$, the bigram response

154 to $A B$ and $B A$ will be identical. Thus, discriminating letter transpositions necessarily 155 requires asymmetric summation in at least one of the neurons. 

parameters for each of the 10 neurons, resulting in $2 \times 10=20$ free parameters. To calculate dissimilarities between a pair of bigrams, we calculated the Euclidean distance between the 10-dimensional response vectors corresponding to the two

160 bigrams. The data collected in the experiment comprised dissimilarities (1/RT) from

1611,176 searches involving all possible pairs of 49 bigrams. To estimate the model parameters, we optimized them to match the observed bigram dissimilarities using standard nonlinear fitting algorithms (see Methods).

This letter model yielded excellent fits to the observed data $(r=0.85, p<$ 0.00005; Figure 3C). To assess whether the model explains all the systematic variance in the data, we calculated an upper bound estimated from the inter-subject consistency (see Methods). This consistency measure ( $r_{\text {data }}=0.90$ ) was close to the model fit, suggesting that the model captured nearly all the systematic variance in the data. As predicted in the schematic figure (Figure 3B), the estimated spatial summation weights were unequal (absolute difference between $w_{1}$ and $w_{2}$, mean $\pm s d$ : $0.07 \pm 0.04$ ). To assess whether this difference is statistically significant, we randomly shuffled the observed dissimilarities and estimated these weights. The absolute difference between shuffled weights was significantly smaller than for the original weights (average absolute difference: $0.03 \pm 0.02 ; p<0.005$, sign-rank test across 10 neurons).

According to an influential account of word reading, specialized detectors are

177 formed for frequently occurring combinations of letters (Dehaene et al., 2005). If this were the case, searches involving frequent bigrams (e.g. TH, ND) or two letter words (e.g. AN, AM) should produce larger model errors compared to infrequent bigrams, 180 since our model does not incorporate any bigram-selective units. Alternatively, if 
bigram discrimination was driven entirely by single letters, we should find no difference

182 in errors. In keeping with this latter prediction, we observed no visually obvious difference in model fits for frequent bigram pairs or word-word pairs compared to other

184 bigram pairs (Figure 3C). To quantify this observation, we asked whether the model

185 error for each bigram pair, calculated as the absolute difference between observed and predicted dissimilarity, covaried with the average bigram frequency of the two bigrams (for both frequent bigrams and words). This revealed a weak negative correlation whereby frequent bigram pairs showed smaller errors $(r=-0.06, p=0.04$ across 1176 bigram pairs). This is the opposite of what would be expected if there were specialized detectors. To further investigate possible bigram frequency effects, we compared the model error for the 20 bigram pairs with the largest mean bigram frequency with the 20 pairs with the lowest mean bigram frequency. This too revealed no systematic difference (mean $\pm \mathrm{sd}$ of residual error: $0.10 \pm 0.08$ for the 20 most frequent bigrams and words; $0.11 \pm 0.09$ for 20 least frequent bigrams; $p=0.80$, ranksum test). Thus, model errors are not systematically different for frequent compared using single neurons tuned to single letters.

\section{Experiment 3: Upright versus inverted bigrams}

In the letter model described above, the response to bigrams is a weighted sum of the single letter responses. As detailed earlier, a critical prediction of this model is that the response to transposed bigrams such as $A B$ \& BA will be different only if the 
expertise can modulate sensitivity to letter transpositions, we reasoned that familiarity might modulate the summation to make it more asymmetric. We therefore predicted

208 that this would make transposed letter searches (with $A B$ as target and $B A$ as

209 distractor, or vice-versa) easier to discriminate in a familiar upright orientation

210 compared to the (unfamiliar) inverted orientation. By contrast, searches involving repeated letter bigrams (with $\mathrm{AA}$ as target and $\mathrm{BB}$ as distractor), which also have a change in two letters, will remain equally easy in both upright and inverted orientations.

214 searches involving upright and inverted bigrams. The essential findings are

215 summarized in Figure 3D. As predicted, subjects discriminated repeated letter bigrams

216 (AA-BB searches) equally well at both upright and inverted orientations, but were

217 substantially faster at discriminating transposed letter pairs (AB-BA searches) in the

218 upright orientation (Figure 3D; for detailed analyses see Section S2). We obtained

219 similar results on comparing upright and inverted trigrams as well (Section S2).

220 We conclude that familiarity leads to asymmetric spatial summation. 
A

\begin{tabular}{|ll|}
\hline AT AT & AT AT \\
AT UT & AT AT \\
AT AT & TA AT \\
AT AT & AT AT \\
\hline
\end{tabular}

B

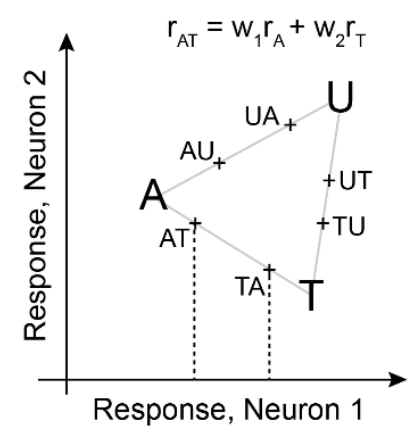

$\mathrm{F}$

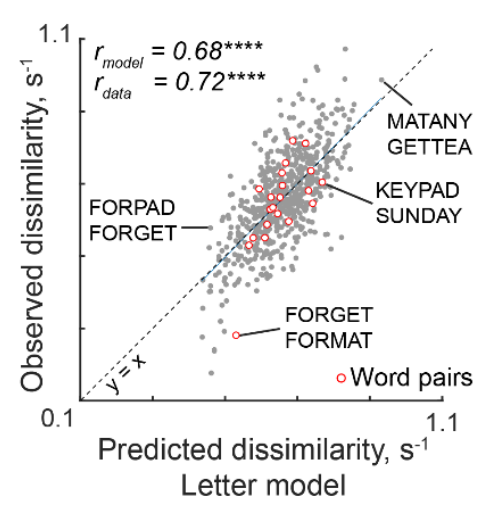

C

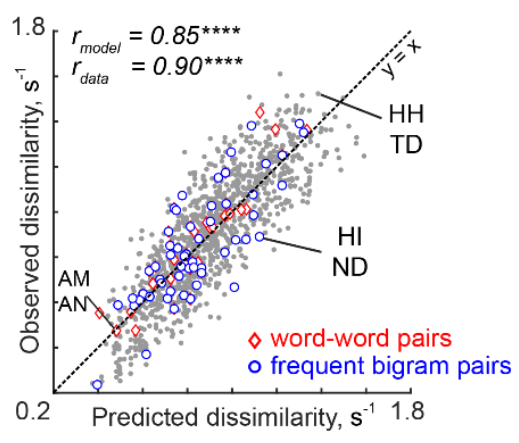

G

$\mathrm{H}$

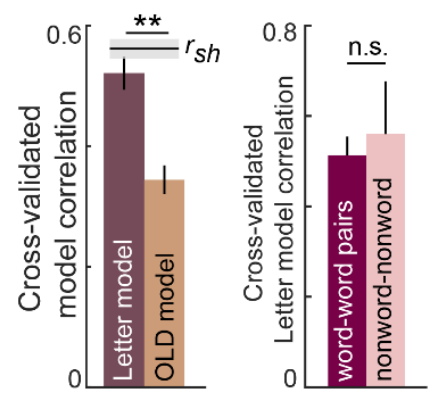

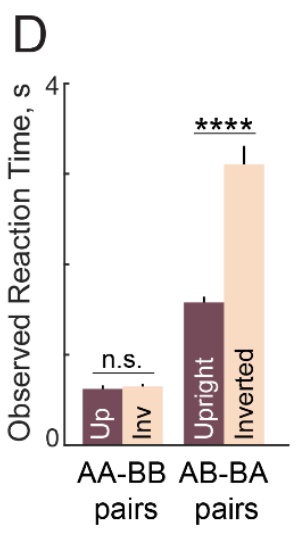

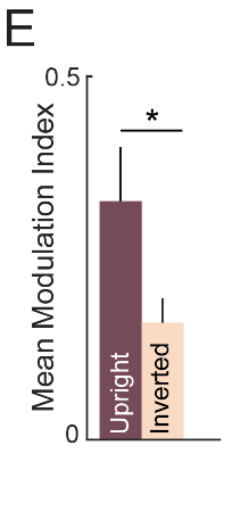

Figure 3. Discrimination of strings is explained using single letters (Expts 2-4)

(A) Example search array with two oddball targets (UT \& TA) among the bigram AT. It can be seen that UT is easier to find than TA, showing that letter substitution causes a bigger visual change compared to transposition.

(B) Schematic diagram of how the bigram response is obtained from letter responses. Consider two neurons selective to single letters $A, T$ \& $U$. These letters can be represented in a $2 \mathrm{D}$ space in which the response to each neuron lies along one axis. For each neuron, we take the response to a bigram to be a weighted sum of the single letter responses. Thus, the bigram response lies along the line joining the two stimuli. Note that the bigrams AT and TA can be distinguished only if there is unequal summation. In the schematic, the first position is taken to have higher magnitude, as a result of which the response to AT is closer to A than to T.

(C) Observed dissimilarities between bigram pairs plotted against predictions of the letter model for word-word pairs (red diamonds), frequent bigram pairs (blue circles) and all other bigram pairs (gray dots), for Experiment 2. Model correlation is shown at the top left, along with the data consistency for comparison. Asterisks indicate the statistical significance of the correlations ${ }^{* * * *}$ is $\left.p<0.00005\right)$.

(D) Average observed search reaction time for upright (dark) and inverted (pale) bigram searches for repeated letter pairs (AA-BB pairs) and transposed letter pairs (AB-BA pairs) in Experiment 3. Asterisks indicate statistical significance of the main effect of orientation in an ANOVA (see text for details; ${ }^{* * * *}$ is $p<0.00005$ ).

(E) Mean modulation index of the summation weights, calculated as |w1-w2|/|w1+w2|, where $\mathrm{w} 1$ and $\mathrm{w} 2$ are the bigram summation weights, averaged across the 10 neurons in the letter model for upright (dark) and inverted (pale) bigrams. The asterisk indicates statistical significance calculated on a sign-rank test comparing the modulation index across 10 neurons $\left({ }^{*}\right.$ is $p<0.05$ ). 
(F) Observed dissimilarities between 6-letter strings in visual search (Experiment 4) plotted against predicted dissimilarities from the single letter model for word-word pairs (red dots) and all other pairs (gray dots). Model correlation is shown at the top left with data consistency for comparison. Asterisks indicate statistical significance of the correlations $\left({ }^{* * *}\right.$ is $\left.p<0.00005\right)$.

(G)Cross-validated model correlation for the letter model (dark) and the Orthographic Levenshtein distance (OLD) model (light). For each model, the cross-validated correlation is the correlation between model predictions trained on one half of the data and the observed response times from the other half. The upper bound on model fits is the split-half correlation $\left(r_{s h}\right)$ shown in black with shaded error bars representing standard deviation across 1000 random splits. The asterisk indicates statistical significance of the comparison obtained by estimating the fraction of bootstrap samples in which the observed difference was violated ( ${ }^{* *}$ is $\left.p<0.005\right)$.

(H) Cross-validated letter model correlation for word-word pairs and nonword-nonword pairs.

\section{Generalization to longer strings}

To investigate whether these results would generalize to longer strings which can contain frequent words, we performed several additional visual search experiments using 3, 4, 5 and 6-letter uppercase strings (Section S4). In Experiment 4, subjects performed visual search involving six-letter strings that were either valid compound words (e.g. FORGET, TEAPOT) or pseudowords (FORPOT, TEAGET). The single letter model yielded excellent fits to the data (Figure 3F). These fits were superior to a widely used measure of string similarity, the Orthographic Levenshtein Distance (OLD) model (Figure 3G). Importantly, the letter model fits were equivalent for both word-word pairs and nonword-nonword pairs (Figure $3 \mathrm{H}$ ). These and other analyses are described in Section S3.

The letter model also yielded excellent fits across all string lengths tested. We also tested lowercase and mixed-case strings because word shape is thought to play a role when letters vary in size or have upward and downward deflections (Pelli and Tillman, 2007). Even here, the letter model, without any explicit representation of overall word shape, was able to accurately predict most of the search performance. These results are detailed in Section S4. 

on dissimilarities between letters presented in isolation. It could be that the representation of a letter within a bigram, although compositional, differs from its representation when seen in isolation. To explore these possibilities we developed an alternate model in which bigram dissimilarities can be predicted using a sum of

291 (unknown) part dissimilarities at different locations. The resulting model, which we denote as the part sum model, yielded comparable fits to the data. It is completely equivalent to the letter model under certain conditions. Unlike the letter model which is nonlinear and could suffer from multiple local minima, the part sum model is linear and its parameters can be estimated uniquely using standard linear regression. Its complexity can be drastically reduced using simplifying assumptions without affecting model fits. These results are detailed in Section S5.

\section{Experiment 5: Lexical decision task}

The above experiments show that discrimination of strings in visual search can be explained by neurons tuned for single letter shape with letter responses that combine linearly. Could the same shape representation drive reading behaviour? We evaluated this possibility through two separate word recognition experiments.

In Experiment 5, we used a widely used paradigm for word recognition, a lexical decision task (Norris, 2013; Grainger, 2018). Subjects had to indicate whether a string of letters is a word or not using a keypress. The words comprised 4, 5 or 6 -letter words and the nonwords consisted of random strings and jumbled words. Subjects were highly accurate in responding to both words and nonwords (mean \pm sd: $96 \pm 2 \%$ for words, $95 \pm 3 \%$ for nonwords). Importantly, their response times across words and

310 nonwords were consistent as evidenced by a significant split-half correlation 
311 (correlation between odd- and even-numbered subjects: $r=0.59$ for words, $r=0.73$

312 for nonwords, $\mathrm{p}<0.00005)$. Since responses in lexical decision tasks are thought to

313 depend on accumulation of evidence towards or against word status (Ratcliff et al.,

314 2004; Ratcliff and McKoon, 2008), we hypothesized that looking at a string of letters

315 will activate the compositional neural code for the string which is then compared to

316 stored patterns corresponding to known words.

317 We started by characterizing response times for words. To depict the

318 systematic variation in word response times, we plotted them in descending order

319 (Figure 4A). Subjects took longer to respond to infrequent words like MALICE

320 compared to frequent words like MUSIC. If the string is a word, the response time will

321 depend on the strength of the stored pattern, which in turn would depend on lexical

322 factors such as word frequency (Ratcliff et al., 2004; Ratcliff and McKoon, 2008).

323 Indeed, response times for words showed a negative correlation with log word

324 frequency $(r=-0.5, p<0.00005$ across 450 words). We also estimated other lexical

325 factors such as the logarithm of the letter frequency (averaged across letters of the

326 string), logarithm of the bigram frequency (averaged across all bigrams in the string),

327 and the number of orthographic neighbours (i.e. number of nearby words), which are

328 all standard measures in linguistic corpora (see Methods).

To avoid overfitting, we trained a model based on each factor on one half of the

330 subjects and tested it on the other half. This cross-validated performance is shown for

331 all lexical factors in Figure 4B. It can be seen that the word frequency is the best

332 predictor of word response times (Figure 4B). To assess whether all lexical factors

333 together predict word response times any better, we fit a combined model in which the

334 word response times are modelled as a linear sum of the four factors. The combined

335 model performance was comparable to the performance of the word frequency model 
alone (Figure 4B). To assess the statistical significance of these results, we performed a bootstrap analysis. On each trial, we trained all models on the dissimilarity obtained from considering only one randomly chosen half of subjects. We calculated the correlation between each model's predictions on the other half of the data, and repeated this procedure 1000 times. Across these samples, the word frequency model performance rarely fell below all other individual models $(p<0.005)$. We conclude that word response times are determined primarily by word frequency. nonword responses are plotted in descending order in Figure 4C. Subjects took longer to respond to jumbled words like PENICL (original word: PENCIL) with fewer

346 transpositions compared to VTAOCE (original word: OCTAVE) with more

347 transpositions. We hypothesized that, if a string is a nonword, the response will be slow if there is a nearby stored pattern corresponding to a word, and fast otherwise (Dufau et al., 2012; Yap et al., 2015). Likewise the response is likely to be faster if the nearest word is highly familiar (i.e. frequent in the lexicon). Specifically, nonword response times will be inversely proportional to the dissimilarity of the nonword to the nearest word (Figure 4D), and also inversely proportional to the frequency of the nearest word (Figure 4D).

To test this prediction, we took the letter model with 10 neurons with single letter tuning and optimized the spatial summation weights to match the reciprocal of the 356 nonword responses for each word length. The model yielded excellent fits to the data $357(r=0.70, p<0.00005 ;$ Figure 4E). This model fit was comparable to the data consistency $\left(r_{\text {data }}=0.84\right)$. Importantly, this model was able to explain classic phenomena in orthographic processing. Specifically, subjects took longer to respond

360 to nonwords obtained by transposing a letter of a word, compared to nonwords 
obtained through letter substitution - these trends were present in the model predictions as well (Figure 4F). Likewise, subjects took longer when the middle letters were transposed compared to when the edge letters were transposed - as did the model predictions (Figure 4F). These effects replicate the classic orthographic processing effects reported across many studies (Grainger et al., 2012; Norris, 2013; Ziegler et al., 2013; Grainger, 2018).

Next we asked whether a widely used measure of orthographic distance could explain the same data. We selected the Orthographic Levenshtein Distance (OLD), in which the net distance between two strings is calculated as the minimum number of

370 letter additions, transpositions and deletions required to transform one string into another. The OLD model yielded relatively poorer predictions of the data $(r=0.36, p$ $<0.00005$; Figure 4G).

We compared the letter model with two alternate models: the OLD model and a model based on lexical factors. The OLD model is as described above. In the lexical model, the nonword response time is modelled as a linear sum of log word frequency, log mean bigram frequency of words, log mean bigram frequency of nonwords, \# orthographic neighbours, log letter frequency. Since all three models have different numbers of free parameters, we compared their performance using cross-validation: we trained each model on one-half of the subjects and evaluated it on the other half

380 of the subjects. The resulting cross-validated model fits are shown in Figure $4 \mathrm{H}$. The 381 letter model outperformed both the OLD model and the lexical model (model correlations: $r=0.56 \pm 0.02,0.33 \pm 0.01$ and $0.35 \pm 0.01$ for the neural, OLD and lexical models; fraction of bootstrap samples with neural $<$ other models: $p<0.005$;

384 Figure $4 \mathrm{H}$ ). To be absolutely certain that the superior fit of the letter model was not 385 simply due to having more free parameters, we compared the lexical model with a 
reduced version of the letter model with only 5 free parameters (SID model; Section

387 S5). Even this reduced model yielded fits were better than the lexical model (SID

388 model correlation: $r=0.48 \pm .02)$. Finally, a combined model - in which the neural and

389 lexical model predictions were linearly combined - proved to explain more variance

390 than either model (Figure $4 \mathrm{H})$.

391 In sum, we conclude that word response times are explained by word frequency

392 and nonword response times are explained by the distance between the nonword and

393 the nearest word calculated using the compositional neural code. As a further test of

394 the ability of this compositional code to explain word reading, we performed an

395 additional experiment in which subjects had to recognize the identity of a jumbled

396 word. Here too, response times were explained best by the letter model compared to

397 lexical and OLD models (Section S6).

398 
A

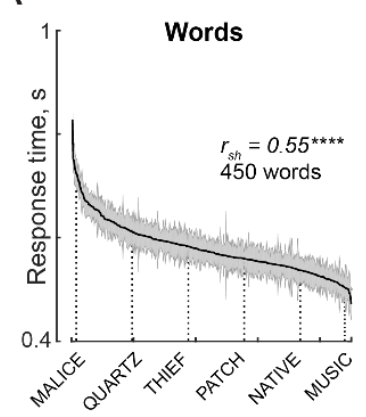

$\mathrm{E}$

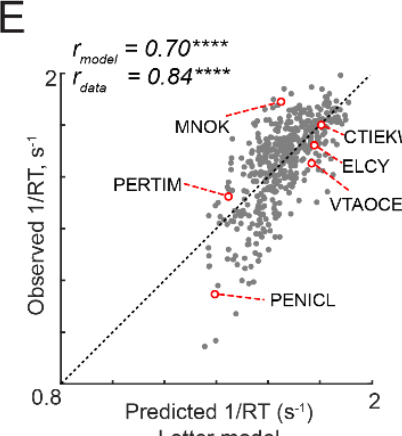

B

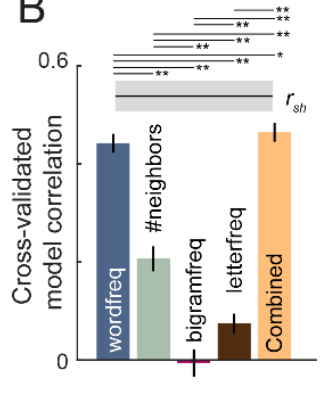

$\mathrm{F}$

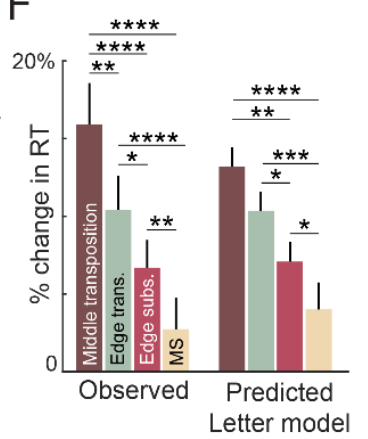

C

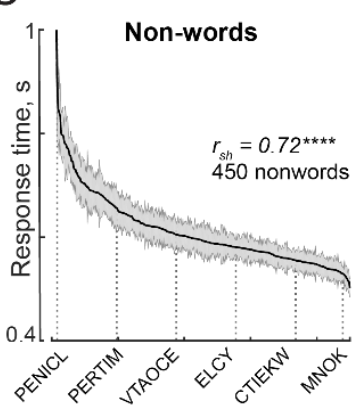

G

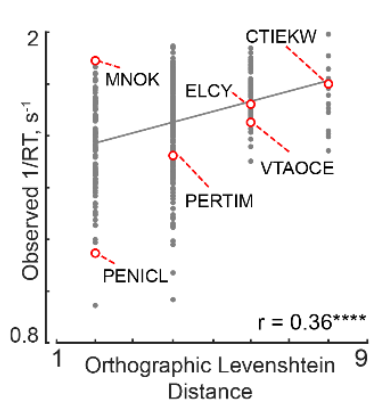

D

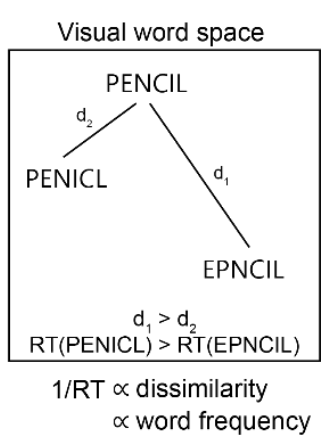

$\mathrm{H}$

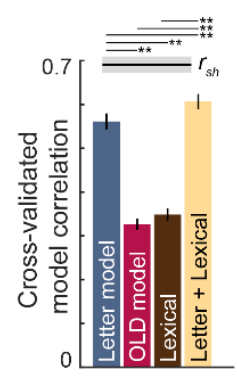

400

401

402

403

404

405

406

407

408

409

410

411

412

413

414

415

416

417

418

419

420

421

422

423

424

425

426

427

428

Figure 4. Lexical decision task behaviour (Experiment 5)

(A) Response times for words in the lexical decision task, sorted in descending order. The solid line represents the mean categorization time for words and the shaded bars represent s.e.m. Some example words are indicated using dotted lines. The split-half correlation between subjects $\left(r_{s h}\right)$ is indicated on the top.

(B) Cross-validated model correlation between observed and predicted word response times across all words for various models: log word frequency (blue), number of orthographic neighbours (orange), log mean bigram frequency (purple), log mean letter frequency (cyan) and a combined model containing all these factors (red). Shaded error bars indicate mean \pm sd of the correlation across 1000 random splits of the observed data. The asterisk indicates statistical significance of the comparison obtained by estimating the fraction of bootstrap samples in which the observed difference was violated ( ${ }^{*}$ is $p<0.05,{ }^{* *}$ is $\left.p<0.005\right)$.

(C) Response times for nonwords in the lexical decision task, sorted in descending order. Conventions as in (A).

(D) Schematic of visual word space, with one stored word (PENCIL) and two nonwords (PENICL \& EPNCIL). We hypothesize that subjects would take longer to categorize a nonword when it is similar to a word, i.e. RT for PENICL would be larger than for EPNCIL. Thus, 1/RT would be proportional to this dissimilarity, and also to word frequency.

(E) Observed reciprocal response times for nonwords in the lexical decision task plotted against letter model predictions fit to the full dataset (450 nonwords). Some example nonwords are depicted.

(F) Percent change in response time (nonword-RT - word-RT)/word-RT for middle \& edge letter transpositions and for middle \& edge substitutions for observed data (left) and for letter model predictions (right). MS: middle substitution. In both cases, asterisks represent statistical significance comparing the means of the corresponding groups using a rank-sum test $\left({ }^{*}\right.$ is $p<0.05,{ }^{* *}$ is $p<0.005$, etc.). 
(G)Observed reciprocal response times plotted against the Orthographic Levenshtein Distance (OLD), a popular model for edit distance between strings.

$(\mathrm{H})$ Cross-validated model correlation between observed and predicted nonword RTs for the letter model, OLD model, lexical model and the combined neural+lexical model. Conventions are as in (B).

\section{Brain activations during lexical decisions (Experiments 6-7)}

The above results show that visual discrimination of strings can be explained using a letter-based compositional neural code, and that dissimilarities calculated using this code can explain human performance on reading tasks. Here, we sought to uncover the brain regions that represent this code and guide eventual lexical decisions.

In Experiment 6, we recorded neural activations using fMRI while subjects performed a lexical decision task. Since lexical decision times for nonwords can be predicted using visual dissimilarity, we performed a separate experiment to directly estimate visual dissimilarities using visual search (Experiment 7; see Methods). Additionally, we estimated the semantic dissimilarity between words in order to compare visual and semantic representations in different ROls (see Methods). Importantly, the visual search and semantic dissimilarities were uncorrelated $(r=0.03$, $\mathrm{p}=0.55)$, thereby allowing us to identify regions with distinct or overlapping visual/semantic representations. The visual and semantic representations are visualized in Section S7.

We identified several possible regions of interest (ROIs) using a combination of functional localizers and anatomical considerations (see Methods). These included the early and mid-level visual areas (V1-V3 \& V4), the object-selective lateral occipital region (LO), and two language areas: the visual word form area (VWFA) which selectively responds to words and a broad region in the temporal gyrus reading 
network (TG). The flattened brain map of a representative subject with these ROls is shown in Figure 5A.

For the main event-related block, subjects had to make a response on each trial to indicate whether a string displayed on the screen was a word or not. The stimuli consisted of 5-letter words, nonwords. Subjects also viewed single letters, to which they had to make no response. Subjects were highly accurate (mean \pm std of accuracy: word frequency $(r=-0.42, p<0.05)$. Likewise, the lexical decision times for nonwords were strongly correlated with the word-nonword dissimilarity measured in visual search in Experiment $7(r=-0.68, p<0.00005)$. These results reconfirm the findings of the previous experiment performed outside the scanner.

We first compared the overall brain activation levels for words, nonwords and

470 letters in each ROI. While V4 showed greater activation for words compared to nonwords, VWFA and TG regions showed greater activation to nonwords compared to words, presumably reflecting greater engagement to discriminate nonwords that are highly similar to words (Section S7). Although the visual regions did not show differential overall activations, there could still be differential activation at the population level for words and nonwords. To assess this possibility, we built linear classifiers to discriminate words from nonwords using the voxel population activity in each ROI. This revealed above-chance classification in all ROls. Further, discriminating words from nonwords was significantly easier for nonwords that were obtained by substituting letters compared to those obtained by transposing letters

480 (Section S7). Correspondingly, in behaviour, subjects were faster at responding to 
substituted nonwords compared to transposed nonwords (response times, mean $\pm \mathrm{sd}$ : these results is presented in Section S7.

\section{Neural correlates of the compositional letter code}

Next we sought to compare the neural representations in each ROI with visual

can be quite distinct, as depicted in Figure 5B: in visual search space, TRAIL and

TRIAL can be quite similar since one is obtained from the other by transposing letters, but the word PATH is quite distinct. By contrast, in semantic space, TRAIL and PATH have similar meanings and usage whereas TRIAL is quite distinct. Indeed, visual search and semantic dissimilarities across words were uncorrelated for the words in experiment $(r=0.03, p=0.55)$.

To investigate these issues, we calculated the neural dissimilarity between each stimulus pair in a given $\mathrm{ROI}$ as the cross-validated Mahalanobis distance between the voxel-wise activations evoked by the two stimuli. We then averaged this dissimilarity across subjects to get an average neural dissimilarity for that $\mathrm{ROI}$. We then compared this neural dissimilarity in each ROI with visual dissimilarities estimated

500 from visual search. This match to visual search dissimilarity is shown in Figure 5C.

501 Among the ROls tested, only the LO dissimilarities showed a significant correlation 502 (correlation between 1024 pairwise dissimilarities involving ${ }^{32} \mathrm{C}_{2}$ words, ${ }^{32} \mathrm{C}_{2}$ nonwords, and 32 word-nonword pairs: $r=0.16, p<0.00005$; Figure $5 C$ ). A searchlight analysis confirmed that the match to visual search dissimilarities was strongest in a region centred around the bilateral LO region (Section S7). Thus, neural dissimilarity 
in the LO region match best with the visual dissimilarities observed in visual search.

We therefore conclude that LO is the likely neural substrate for the compositional letter code.

To further investigate the link between the compositional letter code and the LO

510 representation, we performed several additional analyses. First, we asked whether the neural activation of each voxel in LO could be explained using a linear sum of the single letter activations. Importantly, these model fits were equally good for words and nonwords. This parallels our finding that dissimilarity in visual search was predicted

514 equally well for word-word and nonword-nonword pairs (Figure 3H). Both these results suggest that there are no specialized detectors for letter combinations (Section S7).

516 Second, we confirmed that both the neural tuning for single letters, and the summation weights estimated from the behavioural data in the letter model were qualitatively similar to the observed tuning for single letters and summation weights observed in the voxel activations for the LO region (Section S7).

In sum, we conclude that the LO region is the likely neural substrate for the compositional letter code predicted from behaviour.

Neural basis of semantic space

Next we compared neural representations in each ROI to semantic space. The match to semantic space was significant only in the LO and TG regions (correlation

between 496 pairwise dissimilarities between words: $r=0.18 \pm 0.05$ for LO, $0.22 \pm$ 0.04 for TG; Figure 5D).

The above analysis shows that neural activations in LO are correlated with both compared since they are based on different pairs of stimuli. To investigate whether the 
neural representation in LO matches better with visual search or with semantic space,

532 we compared the match for word-word pairs alone. This revealed no significant

533 difference between the two correlations $(r=0.16 \pm .04$ for LO with visual search, $r=$

$5340.16 \pm 0.05$ for LO with semantic space; $p=0.49$ across 1000 bootstrap samples). We

535 conclude that both LO and TG regions represent semantic space.

536

Neural basis of lexical decisions

If the LO region represents each string (word or nonword) using a compositional

code, then according to the preceding experiments, lexical decisions for words and

540 nonwords must involve some comparison with stored word representations. Recall

541 that lexical decision times for words are correlated with word frequency, and lexical decision times for nonwords are correlated with word-nonword dissimilarity. We therefore asked whether these lexical decision times are correlated with the average

544 activity (across voxels \& subjects) in a given ROI. The resulting correlations are shown

545 in Figure 5F. Across the ROls, only the VWFA showed a consistently positive 546 correlation with lexical decision times for both words and nonwords (Figure 5E). A searchlight analysis confirmed that there was indeed a peak in the correlation with lexical decision times centred on the VWFA, with additional peaks in the parietal and

549 frontal regions (Section S7). This is consistent with word frequency effects observed

550 in these regions (Kronbichler et al., 2004), but we have observed similar effects for

551 nonwords as well. We conclude that lexical decisions are driven by the VWFA. 
A
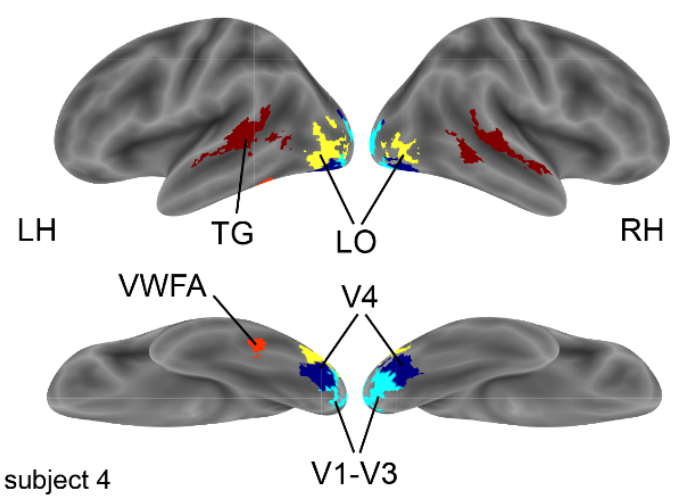

B

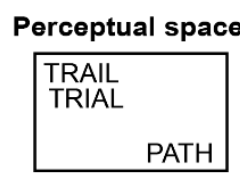

Semantic space

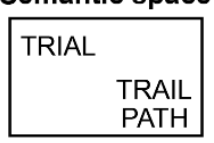

E$$
\text { D }
$$

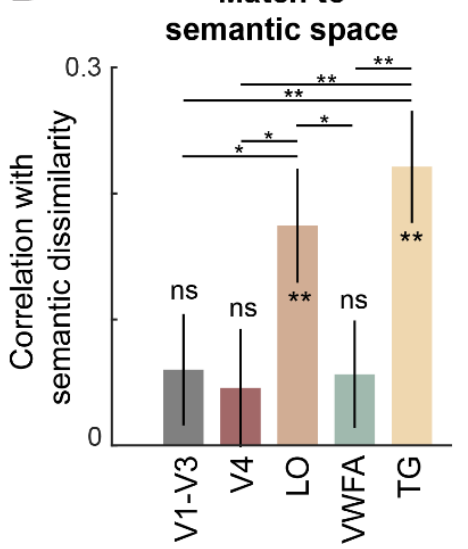

Match to lexical decisions

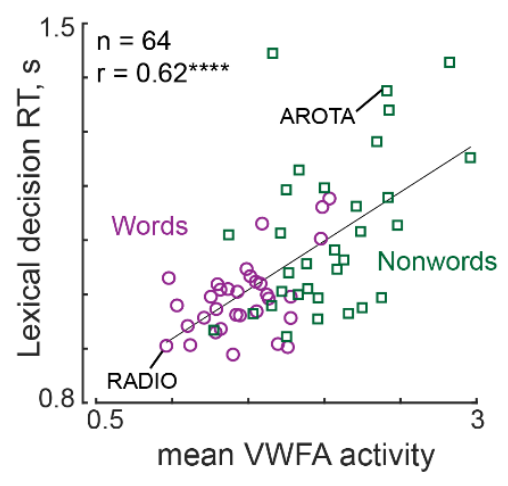

C

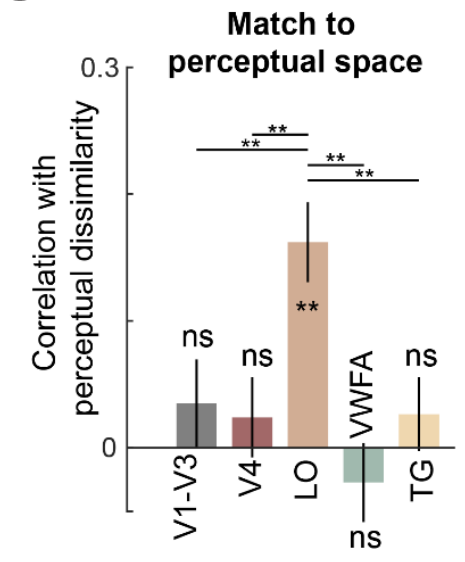

F

Match to lexical decisions

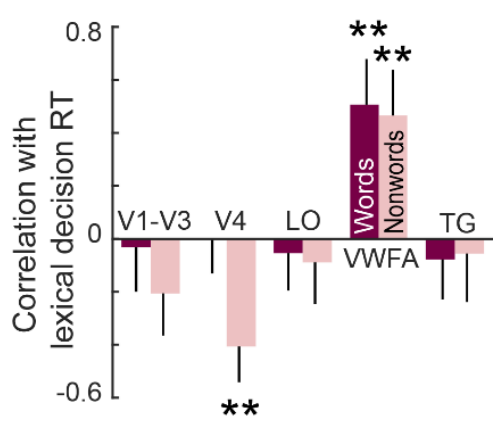

Figure 5. Lexical task fMRI (Experiment 6)

(A) ROls for an example subject, showing V1-V3 (cyan), V4 (blue), LO (yellow), VWFA (red) and TG (maroon).

(B) Example difference between perceptual and semantic spaces. In perceptual space, the representation of TRAIL is closer to its visual similar counterpart TRIAL, whereas in semantic space, its representation is closer to its synonym PATH.

(C) Correlation between neural dissimilarity in each ROI with behavioural dissimilarity for strings (Experiment 7). Error bars indicate standard deviation of the correlation between the group behavioural dissimilarity and ROI dissimilarities calculated repeatedly by resampling of dissimilarity values with replacement across 1000 iterations. Asterisks along the length of each bar indicate statistical significance of the correlation between group behaviour and group ROI dissimilarity ${ }^{* *}$ is $p<$ 0.005 across 1000 bootstrap samples). Horizontal lines indicate the fraction of bootstrap samples in which the observed difference was violated $\left({ }^{*}\right.$ is $p<0.05$, ${ }^{* *}$ is $p<0.005$, etc.). All significant comparisons are indicated.

(D) Correlation between neural dissimilarity in each ROI with semantic dissimilarity for words. Other details are same as in (C).

(E) Correlation between mean VWFA activity (averaged across subjects and voxels) with mean lexical decision time for both words (purple circles) and nonwords (green squares). Each point corresponds to one string and example word and nonword is highlighted. Asterisks indicate statistical significance $\left({ }^{* * *}\right.$ is $\left.p<0.00005\right)$.

(F) Correlation between lexical decision time and mean activity within each ROI separately for words and nonwords. Error bars indicate standard deviation across 1000 bootstrap splits. Asterisks indicate statistical significance $\left(^{* *}\right.$ is $\left.p<0.005\right)$. 


\section{DISCUSSION}

Our main finding is that viewing a string activates a compositional letter code,

consisting of neurons tuned to single letters whose response to longer strings is a linear sum of single letter responses. This code accurately explains human performance on visual search as well as word reading tasks. It is encoded by the LO region in the high-level visual cortex, and subsequent comparisons required for lexical decisions are computed in the VWFA. Below we discuss these findings in relation to the existing literature.

\section{Relation to models of reading}

Our compositional letter code stands in stark contrast to existing models of reading. Existing models of reading assume explicit encoding of letter position and do not account for letter shape (Gomez et al., 2008; Davis, 2010; Norris and Kinoshita, 2012; Norris, 2013). By contrast, our model encodes letter shape explicitly and position

593 implicitly through asymmetric spatial summation. The implicit coding of letter position 594 avoids the complication of counting transpositions (Yarkoni et al., 2008; Yap et al., 2015). Our model can thus easily be extended to any language by simply estimating letter dissimilarities and then estimating the unknown summation weights.

598 plausible and grounded in well-known principles of object representations. The first

599 principle is that images that elicit similar activity across neurons in high-level visual 600 cortex will appear perceptually similar (Op de Beeck et al., 2001; Sripati and Olson,

601 2010a; Zhivago and Arun, 2014). This is non-trivial because it is not necessarily true

602 in lower visual areas or in image pixels (Ratan Murty and Arun, 2015). We have turned 603 this principle around to construct artificial neurons whose shape tuning matches visual 
search. The second principle is that the neural response to multiple objects is typically

Olson, 2010b) that can be biased towards a weighted sum (Ghose and Maunsell,

calculations of letter position in a word, since the neurons in our model only need to be tuned for retinal position. We speculate that these neurons may be tuned not only to retinal position but to the relative size and position of letters, as observed in highlevel visual cortex (Sripati and Olson, 2010a; Vighneshvel and Arun, 2015).

\section{Relation to theories of word recognition}

We have found that lexical decisions for nonwords are driven by the dissimilarity between the viewed string and the nearest word. This idea is consistent with the wellknown Interactive Activation model (McClelland and Rumelhart, 1981; Rumelhart and McClelland, 1982), where viewing a string activates the nearest word representation.

618 However, the Interactive Activation model does not explain lexical decisions or scrambled word reading, and also does not integrate letter shape and position into a unified code. Our findings are consistent with previous work showing that nonword responses are influenced by the number of orthographic neighbours (Yap et al., 2015).

Likewise, we found word frequency to be a major factor influencing lexical decisions,

623 in keeping with previous work (Ratcliff et al., 2004; Dufau et al., 2012; Yap et al., 2015).

624 We have gone further to demonstrate a unified letter-based code that integrates letter shape and position, and localized the underlying neural substrates of the letter code to the LO region, and the comparison process to the VWFA. We propose that the compositional shape code provides a quick match to unscramble a word, failing which subjects may initiate more detailed symbolic manipulation. 

expertise should lead to the formation of specialized bigram detectors (Dehaene et al.,

6312005,$2015 ;$ Grainger, 2018). The presence of these specialized detectors should have

632 caused larger model errors for valid words and frequent n-grams, but we observed no

633 such trend (Figure 3). So what happens to visual letter representations upon expertise

634 with reading? Our comparison of upright and inverted bigrams suggests that reading should increase letter discrimination and increase the asymmetry of spatial summation

636 (Figure 3D,E). This is consistent with differences in letter position effects for symbols 637 and letters (Chanceaux and Grainger, 2012; Scaltritti et al., 2018). We propose that 638 both processes may be driven by visual exposure: repeated viewing of letters makes 639 them more discriminable (Mruczek and Sheinberg, 2005), while viewing letter 640 combinations induces asymmetric spatial weighting. Whether these effects require 641 active discrimination such as letter-sound association training or can be induced even 642 by passive viewing will require comparing letter string discrimination under these 643 paradigms.

\section{Neural basis of word recognition}

Our brain imaging results further elucidate the neural basis of lexical decisions.

647 We have shown that viewing a string activates this compositional letter code in the LO 648 region, and that subsequent comparisons to stored words is driven by the VWFA. We 649 have found that lexical decision response times for both words and nonwords are 650 strongly predicted by the VWFA activity. Since lexical decision times for words are 651 linked to word frequency, this finding implies that VWFA is sensitive to word frequency.

652 This has been confirmed by previous studies (Kronbichler et al., 2004; Dehaene et al., 653 2015). We have also found that VWFA activity is strongly predictive of nonword 
response times, which in turn are modulated by the dissimilarity of the nonword to the

representations (Vinckier et al., 2007) and is modulated by orthographic similarity

658 (Baeck et al., 2015). Our results suggest that VWFA might be involved in comparing

viewed strings with known words. We speculate that differences in this comparison process could explain the contradictory findings about VWFA activation to words and nonwords (Baeck et al., 2015).

\section{Does the compositional letter code explain orthographic processing?} 4F). Its asymmetric spatial weighting favouring the first letter (Section S3), explains the first-letter advantage observed previously (Scaltritti et al., 2018). It also explains why increasing letter spacing can benefit reading in poor readers, presumably because it increases asymmetry in spatial summation (Zorzi et al., 2012).

672 to this neural code, we calculated responses of the letter model trained on data from

673 Experiment 4, and visualized the distances using multidimensional scaling (Figure 6A).

674 It can be seen transposing the edge letters (OFRGET) results in a bigger change than 675 transposing the middle letters (FOGRET), thus explaining many transposed letter 676 effects (Norris, 2013). Likewise, it can be seen that substituting a dissimilar letter

677 (FORXET) leads to a large change compared to substituting a similar letter (FORCET). 678 Replacing $\mathrm{G}$ with $\mathrm{C}$ in FORGET leads to a smaller change than replacing with $\mathrm{X}$, thus 
explaining how priming is stronger when similar letters are substituted (Marcet and 2004; Dehaene et al., 2005).

Finally, as a powerful demonstration of this code, we used it to arbitrarily manipulate reading difficulty along a sentence (Figure 6B), or across multiple transpositions and even number substitutions (Figure 6C). We propose that this compositional neural code can serve as a powerful baseline for the purely visual shape-based representation triggered by viewing words, thereby enabling the study of higher order linguistic influences on reading processes.

\section{Relation between word recognition and reading sentences}

We have shown that word recognition can be explained using a compositional

692 visual code based on single letters. While this is an important first step in

693 understanding how we read single words, reading sentences involves sampling many

694 words with each eye movement (Rayner, 1998). Our ability to sample multiple letters or words at a single glance is limited by two factors. The first is our visual acuity, which reduces with eccentricity. The second is crowding, by which letters become unrecognizable when flanked by other letters - this effect increases with eccentricity 698 (Pelli and Tillman, 2008).

The visual search experiments in our study involved searching for an oddball target (consisting of multiple letters) among multiple distractors. This would most certainly have involved detecting and making saccades to peripheral targets. By contrast, the word recognition tasks in our study involved subjects looking at words presented at the fovea. Our finding that visual search dissimilarity explains word 
704 recognition then implies that shape representations are qualitatively similar in the

705 fovea and periphery. Furthermore, the structure of the letter model suggests a possible

706 mechanistic explanation for crowding. Neural responses might show greater sensitivity

707 to spatial location at the fovea compared to the periphery, leading to more

708 discriminable representations of multiple letters. Alternatively, neural responses to

709 multiple letters might be more predictable from single letters at the fovea but not in the

710 periphery. Both possibilities would predict reduced recognition with closely spaced

711 flankers. Distinguishing these possibilities will require testing neural responses in

712 higher visual areas to single letters and multi-letter strings of both familiar and

713 unfamiliar scripts. Ultimately understanding reading fully will require not only asking

714 how letters combine to form words, but how words combine to form larger units of meaning (Pallier et al., 2011; Nelson et al., 2017).

716 


\begin{tabular}{|cc|}
\hline \multicolumn{2}{|c|}{ FORXET } \\
FORCET: & FGROET \\
FRGT. & FORGET \\
FGEORT. & FOGRET \\
& FORE \\
& .FGORET \\
& FROGET \\
.TGRF & OFRGET \\
\hline
\end{tabular}

B

HUAMN MIDN DOSE NOT RDEA YVERE TRETLE BY FSLTEI HUAMN DNMI DEOS NOT DAER EVREY ETTELR BY ITSLEF ANMHU DINM SOED NOT RDEA ERVEY LTETER BY ITSLEF

\begin{tabular}{|c|}
\hline REAIDNG IS A RECNET CULTRUAL INVENITON \\
\hline REA01NG IS A R3C3NT CUL7UR4L INV3N710N \\
\hline RDNIEAG IS A REENCT CLRTUAUL IONETNIVN \\
\hline
\end{tabular}

(A) Visual word space predicted by the letter model for a word (FORGET) and its jumbled versions. Letter model predictions were based on training the model on compound words (Experiment 4). The plot was obtained by performing multidimensional scaling on the pairwise dissimilarities between strings predicted by the letter model. It can be seen that classic features of orthographic processing are captured by the letter model, including priming effects such as FRGT (green) being more similar to FORGET than TGRF (red).

(B) The letter model can be used to sort jumbled words by their reading difficulty, allowing us to create any desired reading difficulty profile along a sentence. Top row: Sentence with increasing reading difficulty. Middle row: sentence with fluctuating reading difficulty. Bottom row: sentence with decreasing reading difficulty.

(C) The letter model yields a composite measure of reading difficulty that combines letter substitution and transposition effects. Sentences with digit substitutions (second row) can thus be placed along a continuum of reading difficulty relative to other sentences (first, third and fourth rows) with increasing degree of scrambling. 


\section{METHODS}

All subjects had normal or corrected-to-normal vision and gave informed consent to an experimental protocol approved by the Institutional Human Ethics

740 Committee of the Indian Institute of Science. All subjects were fluent English-speaking 741 students at the institute, where English is the medium of instruction. All subjects were multi-lingual and knew at least one other Indian language apart from English.

\section{Experiment 1 - Single letter searches}

Procedure. A total of 16 subjects ( 8 males, $24.4 \pm 2.5$ years) participated in this $\sim 60 \mathrm{~cm}$ away under the control of custom programs written in Psychtoolbox (Brainard, 1997) and MATLAB. In all experiments, we selected sample sizes based on our previous studies which yielded highly consistent data (Agrawal et al., 2019).

Stimuli. Single letter images were created using the Arial font. There were 62 stimuli in all comprising 26 uppercase letters (A-Z), 26 lowercase letters (a-z), and 10 digits (0-9). Uppercase stimuli were scaled to have a height of $1^{\circ}$.

Task. Subjects were asked to perform an oddball search task without any constraints on eye movements. Each trial began with a fixation cross shown for $0.5 \mathrm{~s}$ followed by a $4 \times 4$ search array (measuring $40^{\circ}$ by $25^{\circ}$ ). The search array always contained only one oddball target with 15 identical distractors. Subject were instructed to locate the oddball target as quickly and as accurately as possible, and respond with a key press ('Z' for left, 'M' for right). A red line divided the screen in two halves. The search display was turned off after the response or after 10 seconds, whichever was sooner. All stimuli were presented in white against a black background. Incorrect or missed trials 
were repeated after a random number of other trials. Subjects completed a total of

For each search pair, the oddball target appeared equally often on the left and right sides so as to avoid creating any response bias. Only correct responses were considered for further analysis. The main experiment was preceded by 20 practice trials involving unrelated stimuli.

Data Analysis. Subjects were highly accurate on this task (mean \pm std: $98 \pm 1 \%$ ). function, MATLAB R2018a). This function removes any value greater than three scaled absolute deviations away from the median, and was applied to each search pair separately. This step removed $6.8 \%$ of the response time data.

\section{Estimation of single letter tuning using multidimensional scaling}

To estimate neural responses to single letters from the visual search data, we search time for each letter pair by averaging across subjects and trials. We then converted this search time (RT) into a distance measure by taking its reciprocal (1/RT).

778 This is a meaningful measure because it represents the underlying rate of evidence accumulation in visual search (Sunder and Arun, 2016), behaves like a mathematical distance metric (Arun, 2012) and combines linearly with a variety of factors (Pramod and Arun, 2014, 2016; Sunder and Arun, 2016). Next we took all pairwise distances

782 between letters and performed MDS to embed letters into $\mathrm{n}$ dimensions, where we

783 varied $\mathrm{n}$ from 1 to 15 . This yielded $\mathrm{n}$-dimensional coordinates corresponding to each 784 letter, whose distances matched best with the observed distances. We then took the 
activation of each letter along a given dimension as the response of a single neuron. letter responses of 10 neurons. We obtained qualitatively similar results on varying this number of dimensions.

\section{Estimation of data reliability}

To obtain upper bounds on model performance, we reasoned that any model correlation $r_{s h}$. This process was repeated 100 times to obtain the mean and standard deviation of the split-half correlation. However when a model is trained on all the data, the upper bound will be larger than the split-half correlation. We obtained this upper

\section{Experiment 2 - Bigram searches}

801

A total of 8 subjects ( 5 male, aged $25.6 \pm 2.9$ years) took part in this experiment.

802 We chose seven uppercase letters ( $A, D, H, I, M, N, T)$ and combined them in all possible ways to obtain 49 bigram stimuli. These letters were chosen to maximise the number of two-letter words e.g. HI, IT, IN, AN, AM, AT, AD, AH, and HA. Letters measured $3^{\circ}$ along the longer dimension. Subjects completed 2352 correct trials $\left({ }^{49} \mathrm{C}_{2}\right.$

806 search pairs $\times 2$ repetitions). All other details were identical to Experiment 1.

807 Letter/Bigram frequencies were obtained from an online database (http://norvig.com/mayzner.html). 
809 Data Analysis. Subjects were highly accurate on this task (mean \pm std: $97.6 \pm 1.8 \%$ ).

810 Outliers in the reaction times were removed using built-in routines in MATLAB (isoutlier

811 function, MATLAB R2018a). This step removed $8 \%$ of the response time data.

812

\section{Estimating letter model parameters from observed dissimilarities}

The total dissimilarity between two bigrams in the letter model is calculated by

815 calculating the average dissimilarity across all neurons. For each neuron, the dissimilarity between bigrams $A B$ \& $C D$ is given by:

$$
d(A B, C D)=\left|r_{A B}-r_{C D}\right|=\left|\left(w_{1} r_{A}+w_{2} r_{B}\right)-\left(w_{1} r_{C}+w_{2} r_{D}\right)\right|
$$

where $r_{A}, r_{B}, r_{C}$ and $r_{D}$ are the responses of the neuron to individual letters $\mathrm{A}, \mathrm{B}$,

819 C and D respectively (derived from single letter dissimilarities), and $w_{1}, w_{2}$ are the spatial summation weights for the first and second letters of the bigram. Note that $w_{1}, w_{2}$ are the only free parameters for each neuron. minimize the squared error between the observed and predicted dissimilarity. This

824 adjustment was done using standard gradient descent methods starting from randomly 825 initialized weights (nlinfit function, MATLAB R2018a). We followed a similar approach for experiments involving longer strings.

\section{Experiment 3 - Upright and inverted bigrams}

Methods. A total of 8 subjects ( 6 males, aged $24 \pm 1.5$ years) participated in this

830 experiment. Six uppercase letters: $A, L, N, R, S$, and $T$ were combined in all pairs to

831 form a total of 36 stimuli. These uppercase letters were chosen because their images 
832

833

834

835

836

837

838

839

840

841

842

843

844

845

846

847

848

849

850

851

852

853

854

855

change when inverted (as opposed to letters like $\mathrm{H}$ that are unaffected by inversion), and were chosen to maximize the occurrence of frequent bigrams. The same stimuli were inverted to create another set of 36 stimuli. Detailed analyses for this experiment are presented in Section S2.

\section{Experiment 4 - Compound words}

A total of 8 subjects ( 4 female, aged $25 \pm 2.5$ years) participated. Twelve 3 letter words were chosen: ANY, FOR, TAR, KEY, SUN, TEA, ONE, MAT, GET, PAD, DAY, POT. Each word was jumbled to obtain twelve 3-letter nonwords containing the same letters. The 12 words were combined to form 36 compound words (shown in Section S3), such that they appeared equally on the left and right half of the compound words. Detailed analyses for this experiment are included in Section S3.

Calculation of orthographic Levenshtein distance: For each search pair, we estimated the OLD metric using built-in MATLAB function "editdistance". This function estimates the number of insertions, deletions, or substitutions are required to convert one string to other. In this study, the substitution cost has a value of 2. We obtained qualitatively similar results with other choices of substitution cost.

\section{Experiment 5 - Lexical decision task}

Procedure. A total of 16 subjects (9 male, aged $24.8 \pm 2.1$ years) participated in this task as well as the jumbled word task.

Stimuli. The stimuli comprised 450 words +450 nonwords. The nonwords were either random strings or made by modifying the 450 words in some way, as detailed in the table below. 


\begin{tabular}{|l|l|c|c|c|c|}
\hline & \multicolumn{1}{|c|}{ Variations of word ABCDE } & $\begin{array}{c}4 \\
\text { letter } \\
\text { words }\end{array}$ & $\begin{array}{c}5 \\
\text { letter } \\
\text { words }\end{array}$ & $\begin{array}{c}6 \\
\text { letter } \\
\text { words }\end{array}$ & Total \\
\hline 1) & Edge transpositions: BACDE or ABCED & 15 & 15 & 20 & 50 \\
\hline 2) & Middle transposition: ACBDE or ABDCE & 15 & 15 & 20 & 50 \\
\hline 3) & 2 step edge transposition: CBADE or ABEDC & 0 & 20 & 30 & 50 \\
\hline 4$)$ & 2 step middle transposition: ADCBE & 0 & 20 & 30 & 50 \\
\hline 5) & Random transposition: CDABE, ACDBE, etc. & 25 & 35 & 40 & 100 \\
\hline 6) & Edge Substitution: MZCDE or ABCMZ & 15 & 15 & 20 & 50 \\
\hline 7) & Middle Substitution: ABMZE & 15 & 15 & 20 & 50 \\
\hline 8) & $\begin{array}{l}\text { Random substitution and permutation: } \\
\text { MACZE, AMDEZ, etc. }\end{array}$ & 15 & 15 & 20 & 50 \\
\hline & \multicolumn{1}{|c|}{ Total } & 100 & 150 & 200 & 450 \\
\hline
\end{tabular}

Table 1: Non-word stimuli in lexical decision task (Experiment 5).

858 Task. Each trial began a fixation cross shown for $0.75 \mathrm{~s}$ followed by a letter string for

$8590.2 \mathrm{~s}$ after which the screen went blank. The trial ended either with the subject's response or after at most 3 s. Subjects were instructed to press 'Z' for words and 'M'

861 for nonwords as quickly and accurately as possible. All stimuli were presented at the centre of the screen and were white letters against a black background. Before starting the main task, subjects were given 20 practice trials using other words and nonwords not included in the main experiment.

865

Data Analysis. Some nonwords were removed from further analysis due to low words and nonwords (mean \pm std of accuracy: $96 \pm 2 \%$ for words, $95 \pm 3 \%$ for nonwords). Outliers in the reaction times were removed using built-in routines in MATLAB (isoutlier function, MATLAB R2018a). 
876 Experiment 6 (Lexical Decision Task - fMRI)

A total of 17 subjects (10 males, $25 \pm 4.2$ years) participated in this experiment.

All subjects were screened for safety and comfort beforehand to avoid adverse

879 outcomes in the scanner.

880

Stimuli: The functional localizer block included English words, objects, scrambled words, and scrambled objects. In each run, 14 images were randomly selected from a pool of images. The English words list comprised of 90 five-letter words. Each word was divided into grids of dimension $9 \times 3$. Scrambled words were generated by randomly shuffling the grids. Object pool comprised of 80 man-made objects. To generate scrambled objects, the phase of the Fourier transformed images was scrambled and then reconstructed back using inverse Fourier transform. The object images were about $4.5^{\circ}$ along the longer dimension and the height of the word stimuli subtended $2^{\circ}$ of visual angle. words and 32 nonwords formed using these single letters). The stimulus set comprised of 64 five-letter words and nonwords. The words were chosen from a wide range of

892 frequency of occurrence and the nonwords were created by manipulating the chosen

893 words i.e. They were: 1) 8-middle transposed version of words, 2) 8-edge transposed 894 version of words, 3) 8-middle substituted version of words, and 4) 8-edge substituted version of words. The stimuli subtended $2^{\circ}$ in height, which was the same as in the

896 localizer block. All stimuli were presented as white against a black background.

897 Procedure: In the localizer block, a total of 16 images were presented for $0.8 \mathrm{~s}$ with an 898 inter stimulus interval of $0.2 \mathrm{~s}$. There were 14 unique stimuli and 2 of them repeated 899 at random time point, in which subjects performed one-back task. Each block ended 
with a blank screen with fixation cross present for $4 \mathrm{~s}$. Thus, each block lasted $20 \mathrm{~s}$.

901 Each block was repeated thrice in each run.

902

In the event-related design block, an image was presented at the centre of the

903

screen for $300 \mathrm{~ms}$ followed by 3.7 s of blank screen with a fixation cross. In a run, all

904

74 stimuli were presented once along with 16 trials of fixation cross to jitter inter

905

stimulus interval. Hence there were a total of 92 trials including $4 s$ fixation trials at the

906 start and end of each run. Each run lasted 376 s. Subjects performed lexical decision

907 task only on strings and were instructed to not press any key for single letters. Overall, subjects completed 2 runs of localizer block, 8 runs of event block and a structural scan block.

910 Data acquisition: Subjects viewed images in a mirror-based projection system.

911 Functional MRI data was acquired using a 32-channel head coil on a 3T Siemens

912 Skyra scanner at HealthCare Global Hospital, Bengaluru. Functional scans were 913 performed using a T2*-weighted gradient-echo-planar imaging sequence with the

914 following parameters: $\mathrm{TR}=2 \mathrm{~s}, \mathrm{TE}=28 \mathrm{~ms}$, flip angle $=79^{\circ}$, voxel size $=3 \times 3 \times 3 \mathrm{~mm}^{3}$,

915 field of view $=192 \times 192 \mathrm{~mm}^{2}$, and 33 axial-oblique slices covering the whole brain.

916 Anatomical scans were performed using T1-weighted images with the following

917 parameters: $\mathrm{TR}=2.30 \mathrm{~s}, \mathrm{TE}=1.99 \mathrm{~ms}$, flip angle $=9^{\circ}$, voxel size $=1 \times 1 \times 1 \mathrm{~mm}^{3}$, field

918 of view $=256 \times 256 \times 176 \mathrm{~mm}^{3}$.

919 Data preprocessing: All raw fMRI data were processed using custom built MATLAB 920 scripts that depended on $\quad$ SPM 12 toolbox

921 (https://www.fil.ion.ucl.ac.uk/spm/software/spm12/). Raw images were realigned, 922 slice-time corrected, co-registered with the anatomical image, segmented, and finally 923 normalized to the MNI305 anatomical template. The results were qualitatively similar 
without normalization. Smoothing operation was performed only on functional localizer normalization, the data was preprocessed using GLMdenoise v1.4 (Kay et al., 2013). This step improved the signal-to-noise ratio in the data by regressing out the noise pattern common across all the voxels in the brain. The noise pattern is estimated from voxels unrelated to the task. The activity corresponding to each condition was estimated by modelling the denoised data using a generalized linear model (GLM) in SPM after removing the low frequency drift using a high-pass filter with a cutoff at 128s. The event block data was modelled using 89 regressors $(74$ stimuli +1 fixation

$934+6$ motion regressors +8 runs). The localizer block data was modelled using 13 regressors ( 4 stimuli +1 fixation +6 motion regressors +2 runs) .

ROI definitions: All the regions of interest $(\mathrm{ROI})$ were defined using functional localizer while taking the anatomical location into consideration. Early visual area was defined as the region that responds more to the scrambled object than fixation cross. This functional region was further parsed into V1-V3 and V4 using an anatomical mask from SPM anatomy toolbox (Eickhoff et al., 2005). Lateral Occipital (LO) region was defined as a group of voxels that responded more to objects than scrambled objects.

942 The voxels in the LO region was restricted to Inferior Temporal Gyrus, Inferior Occipital

943 Gyrus, and Middle Occipital Gyrus. These anatomical regions were obtained from

944 Tissue Probability Map (TPM) labels in SPM 12. Visual Word Form Area (VWFA) was

945 defined as a region that responded more for words than scrambled words within

946 fusiform Gyrus. The activity for known words was also higher in Superior and Middle

947 Temporal regions. These groups of voxels were grouped under Temporal Gyrus (TG)

948 label. For each contrast, voxel-level threshold of $p<0.001$ (uncorrected) or cluster 
949 level threshold $p<0.05$ (FWE correction) was used to obtain a contiguous region. For

950 one subject, very few VWFA voxels cross the pre-specified threshold. Hence, the

951 threshold was lowered to $p=0.1$ (uncorrected). The VWFA voxels were restricted to

952 top-40 voxels (based on T-value in the function localizer contrast). All these regions

953 were visualized on the cortical surface using BSPMVIEW toolbox

954 (http://www.bobspunt.com/bspmview/).

956 Calculation of neural dissimilarity (fMRI). For each ROI and subject, the pair-wise

957 dissimilarity between any two image pairs was computed using the cross-validated

958 Mahalanobis distance in the RSA toolbox (Nili et al., 2014). Outliers in dissimilarity

959 values across subjects were removed using built-in routines in MATLAB (isoutlier

960 function, MATLAB R2018a). The median dissimilarity across all the subjects was

961 considered for further analysis. We obtained qualitatively similar results for other

962 distance measures.

963

964 Calculation of semantic dissimilarity. The semantic distance between every pair of

965 words was computed as the cosine distance between the GloVe (Pennington et al.,

966 2014) feature vectors activated by the two words, using the MATLAB function

967 word2vec. These features are based on the co-occurrence statistics of words in a large

968 text corpus, and therefore reflect semantic dissimilarity rather than visual dissimilarity.

970 Experiment 7 (5-letter string searches)

A total of 11 subjects ( 6 males, $26 \pm 2.7$ years) participated in this experiment,

972 of which xx also participated in Experiment 6. Stimuli were identical to Experiment 6, 
973 except that they were scaled down to a height of $1^{\circ}$ to allow placement in a visual

974 search array. Subjects performed a total of 2048 correct trials $\left({ }^{32} \mathrm{C}_{2}\right.$ search pairs $\times 2$

975 conditions (words and nonwords) +32 word-nonword pairs $\times 2$ repetitions). All trials

976 were interleaved, and incorrect/missed trials appeared randomly later in the task but

977 were not analyzed. All other details were identical to Experiment 1.

978

979 Data Analysis. Subjects were highly accurate on this task (mean \pm std: $98.6 \pm 1 \%$ ).

980 Outliers in the reaction times were removed using built-in routines in MATLAB (isoutlier

981 function, MATLAB R2018a). This step removed 7\% of the response time data. 
982

983

984

985

986

987

988

989

990

991

992

993

994

995

996

997

998

999

1000

1001

1002

1003

1004

1005

1006

1007

1008

1009

1010

1011

1012

1013

1014

1015

1016

1017

1018

1019

1020

1021

1022

1023

1024

1025

1026

1027

1028

1029

1030

1031

\section{REFERENCES}

Agrawal A, Hari KVS, Arun SP (2019) Reading Increases the Compositionality of Visual Word Representations. Psychol Sci 30:1707-1723.

Arun SP (2012) Turning visual search time on its head. Vision Res 74:86-92.

Baeck A, Kravitz D, Baker C, Op de Beeck HP (2015) Influence of lexical status and orthographic similarity on the multi-voxel response of the visual word form area. Neuroimage 111:321-328.

Bao P, Tsao DY (2018) Representation of multiple objects in macaque categoryselective areas. Nat Commun 9:1774.

Brainard DH (1997) The Psychophysics Toolbox. Spat Vis 10:433-436.

Chanceaux M, Grainger J (2012) Serial position effects in the identification of letters, digits, symbols, and shapes in peripheral vision. Acta Psychol (Amst) 141:149_ 158.

Davis CJ (2010) The spatial coding model of visual word identification. Psychol Rev 117:713-758.

Dehaene S, Cohen L, Morais J, Kolinsky R (2015) Illiterate to literate: behavioural and cerebral changes induced by reading acquisition. Nat Rev Neurosci 16:234-244.

Dehaene S, Cohen L, Sigman M, Vinckier F (2005) The neural code for written words: a proposal. Trends Cogn Sci 9:335-341.

Dehaene S, Pegado F, Braga LW, Ventura P, Nunes Filho G, Jobert A, DehaeneLambertz G, Kolinsky R, Morais J, Cohen L (2010) How learning to read changes the cortical networks for vision and language. Science (80- ) 330:13591364.

Dufau S, Grainger J, Ziegler JC (2012) How to say "No" to a nonword: A leaky competing accumulator model of lexical decision. J Exp Psychol Learn Mem Cogn 38:1117-1128.

Eickhoff SB, Stephan KE, Mohlberg H, Grefkes C, Fink GR, Amunts K, Zilles K (2005) A new SPM toolbox for combining probabilistic cytoarchitectonic maps and functional imaging data. Neuroimage 25:1325-1335.

Ghose GM, Maunsell JH (2008) Spatial summation can explain the attentional modulation of neuronal responses to multiple stimuli in area v4. J Neurosci 28:5115-5126.

Gomez P, Ratcliff R, Perea M (2008) The overlap model: a model of letter position coding. Psychol Rev 115:577-600.

Grainger J (2018) Orthographic processing: A 'mid-level' vision of reading: The 44th Sir Frederic Bartlett Lecture. Q J Exp Psychol 71:335-359.

Grainger J, Dufau S, Montant M, Ziegler JC, Fagot J (2012) Orthographic processing in baboons (Papio papio). Science (80- ) 336:245-248.

Grainger J, Whitney C (2004) Does the huamn mnid raed wrods as a wlohe? Trends Cogn Sci 8:58-59.

Kay KN, Rokem A, Winawer J, Dougherty RF, Wandell BA (2013) GLMdenoise: A fast, automated technique for denoising task-based fMRI data. Front Neurosci 7:1-15.

Kronbichler M, Hutzler F, Wimmer H, Mair A, Staffen W, Ladurner G (2004) The visual word form area and the frequency with which words are encountered: evidence from a parametric fMRI study. Neuroimage 21:946-953.

Lehky SR, Tanaka K (2016) Neural representation for object recognition in inferotemporal cortex. Curr Opin Neurobiol 37:23-35.

Marcet A, Perea M (2017) Is nevtral NEUTRAL? Visual similarity effects in the early 
phases of written-word recognition. Psychon Bull Rev 24:1180-1185.

McClelland JL, Rumelhart DE (1981) An interactive activation model of context effects in letter perception: I. An account of basic findings. Psychol Rev 88:375407.

Mruczek REB, Sheinberg DL (2005) Distractor familiarity leads to more efficient visual search for complex stimuli. Percept Psychophys 67:1016-1031.

Nelson MJ, EI Karoui I, Giber K, Yang X, Cohen L, Koopman H, Cash SS, Naccache L, Hale JT, Pallier C, Dehaene S (2017) Neurophysiological dynamics of phrase-structure building during sentence processing. Proc Natl Acad Sci:201701590.

Nili H, Wingfield C, Walther A, Su L, Marslen-Wilson W, Kriegeskorte N (2014) A toolbox for representational similarity analysis. PLoS Comput Biol 10:e1003553.

Norris D (2013) Models of visual word recognition. Trends Cogn Sci 17:517-524.

Norris D, Kinoshita S (2012) Reading through a noisy channel: why there's nothing special about the perception of orthography. Psychol Rev 119:517-545.

Op de Beeck H, Wagemans J, Vogels R (2001) Inferotemporal neurons represent low-dimensional configurations of parameterized shapes. Nat Neurosci 4:12441252.

Pallier C, Devauchelle A-D, Dehaene S (2011) Cortical representation of the constituent structure of sentences. Proc Natl Acad Sci U S A 108:2522-2527.

Pelli DG, Tillman K a (2008) The uncrowded window of object recognition. Nat Neurosci 11:1129-1135.

Pelli DG, Tillman KA (2007) Parts, wholes and context in reading: A triple dissociation. PLoS One 2:e680.

Pennington J, Socher R, Manning CD (2014) GloVe: Global Vectors for Word Representation. In: Empirical Methods in Natural Language Processing (EMNLP), pp 1532-1543.

Perea M, Duñabeitia JA, Carreiras M (2008) R34D1NG W0RD5 W1TH NUMB3R5. J Exp Psychol Hum Percept Perform 34:237-241.

Perea M, Panadero V (2014) Does viotin activate violin more than viocin? On the use of visual cues during visual-word recognition. Exp Psychol 61:23-29.

Pramod RT, Arun SP (2014) Features in visual search combine linearly. J Vis 14:120.

Pramod RT, Arun SP (2016) Object attributes combine additively in visual search. J Vis 16:8.

Pramod RT, Arun SP (2018) Symmetric Objects Become Special in Perception Because of Generic Computations in Neurons. Psychol Sci 29:95-109.

Ratan Murty NA, Arun SP (2015) Dynamics of 3D view invariance in monkey inferotemporal cortex. J Neurophysiol 113:2180-2194.

Ratcliff R, Gomez P, McKoon G (2004) A diffusion model account of the lexical decision task. Psychol Rev 111:159-182.

Ratcliff R, McKoon G (2008) The Diffusion Decision Model: Theory and Data for Two-Choice Decision Tasks. Neural Comput 20:873-922.

Rawlinson GE (1976) The significance of letter position in word recognition.

Rayner K (1998) Eye movements in reading and information processing: 20 years of research. Psychol Bull 124:372-422.

Rayner K, White SJ, Johnson RL, Liversedge SP (2006) Raeding wrods with jubmled lettres: There is a cost. Psychol Sci 17:192-193.

Rumelhart DE, McClelland JL (1982) An interactive activation model of context effects in letter perception: Part 2. The contextual enhancement effect and some 
1082

1083

1084

1085

1086

1087

1088

1089

1090

1091

1092

1093

1094

1095

1096

1097

1098

1099

1100

1101

1102

1103

1104

1105

1106

1107

1108

1109

1110

1111

1112

1113

1114

1115

1116

1117

1118

1119

1120

1121

1122

1123

tests and extensions of the model. Psychol Rev 89:60-94.

Scaltritti M, Dufau S, Grainger J (2018) Stimulus orientation and the first-letter advantage. Acta Psychol (Amst) 183:37-42.

Sripati AP, Olson CR (2010a) Global Image Dissimilarity in Macaque Inferotemporal Cortex Predicts Human Visual Search Efficiency. J Neurosci 30:1258-1269.

Sripati AP, Olson CR (2010b) Responses to compound objects in monkey inferotemporal cortex: the whole is equal to the sum of the discrete parts. $J$ Neurosci 30:7948-7960.

Sunder S, Arun SP (2016) Look before you seek: Preview adds a fixed benefit to all searches. J Vis 16:3.

Vighneshvel T, Arun SP (2015) Coding of relative size in monkey inferotemporal cortex. J Neurophysiol 113:2173-2179.

Vinckier F, Dehaene S, Jobert A, Dubus JP, Sigman M, Cohen L (2007) Hierarchical Coding of Letter Strings in the Ventral Stream: Dissecting the Inner Organization of the Visual Word-Form System. Neuron 55:143-156.

Yap MJ, Sibley DE, Balota DA, Ratcliff R, Rueckl J (2015) Responding to nonwords in the lexical decision task: Insights from the english Lexicon project. J Exp Psychol Learn Mem Cogn 41:597-613.

Yarkoni T, Balota D, Yap M (2008) Moving beyond Coltheart's N: a new measure of orthographic similarity. Psychon Bull Rev 15:971-979.

Zhivago KA, Arun SP (2014) Texture discriminability in monkey inferotemporal cortex predicts human texture perception. J Neurophysiol 112:2745-2755.

Ziegler JC, Hannagan T, Dufau S, Montant M, Fagot J, Grainger J (2013)

Transposed-Letter Effects Reveal Orthographic Processing in Baboons. Psychol Sci 24:1609-1611.

Zoccolan D, Cox DD, DiCarlo JJ (2005) Multiple Object Response Normalization in Monkey Inferotemporal Cortex. J Neurosci 25:8150-8164.

Zorzi M, Barbiero C, Facoetti A, Lonciari I, Carrozzi M, Montico M, Bravar L, George F, Pech-Georgel C, Ziegler JC (2012) Extra-large letter spacing improves reading in dyslexia. Proc Natl Acad Sci U S A 109:11455-11459.

\section{ACKNOWLEDGEMENTS}

Funding. This research study was funded by Intermediate \& Senior Fellowships (Grant Numbers 500027/Z/09/Z and IA/S/17/1/503081) from the Wellcome Trust/DBT India Alliance and the DBT-IISc partnership program (to SPA).

Author contributions. AA, KVSH \& SPA designed experiments; AA collected data; AA \& SPA analysed and interpreted the data; SPA wrote the manuscript with inputs from AA \& KVSH.

Competing interests. The authors declare no competing interests.

Data and code availability. Data and code necessary to reproduce the results are available at https://osf.io/384zw/ 


\section{APPENDIX}

For

\section{A compositional letter code in high-level visual cortex} explains how we read jumbled words

\section{CONTENTS}

Section A1. Additional analyses for Experiment 1 (single letters)

Section A2. Upright and inverted bigrams and trigrams (Expts 3 \& S1)

Section A3. Compound words (Experiment 4)

Section A4. Experiments with longer strings (Expts S2-S5)

Section A5. Estimating letter dissimilarities from bigram dissimilarities

Section A6. Jumbled word reading (Expt S6)

Section A7. Additional analyses for Experiment 5 (lexical task)

Section A8. Additional analyses for LDT fMRI (Expts 6-7)

References 
The results in the main text were presented for uppercase English letters (Figure 2), but in Experiment 1 we also collected visual search data for all pairs of English letters and numbers $(n=62$ characters in all, comprising 26 uppercase +26 lowercase +10 numbers). We did so in order to predict the visual dissimilarity between letter strings containing both mixed case letters as well as numbers.

To visualize the dissimilarity relations between the 62 characters used, we performed multidimensional scaling. In the resulting plot (Figure S1A), nearby characters represent hard searches. A number of interesting patterns can be seen: letters like $C, G, Q, O$ are nearby which is expected given their shared curvatures. Letter pairs such as $(\mathrm{M}, \mathrm{W})$ and number pairs such as $(6,9)$ are similar due to mirror confusion (Vighneshvel and Arun, 2013).

Next, we investigated the degree to which the observed pairwise dissimilarities are captured by the multidimensional embedding as a function of the number of dimensions. In the resulting plot (Figure S1B), it can be seen that nearly $89 \%$ of the variance is captured by 10 dimensions as before, which reaches roughly the reliability of the dissimilarity data itself. For the analyses involving mixed case searches or fewer searches, we took a total of 6 neurons for the letter model, which explain $87.7 \%$ of the variance in the pairwise dissimilarities.

A

Visual search space (Uppercase/lowercase letters and numbers)

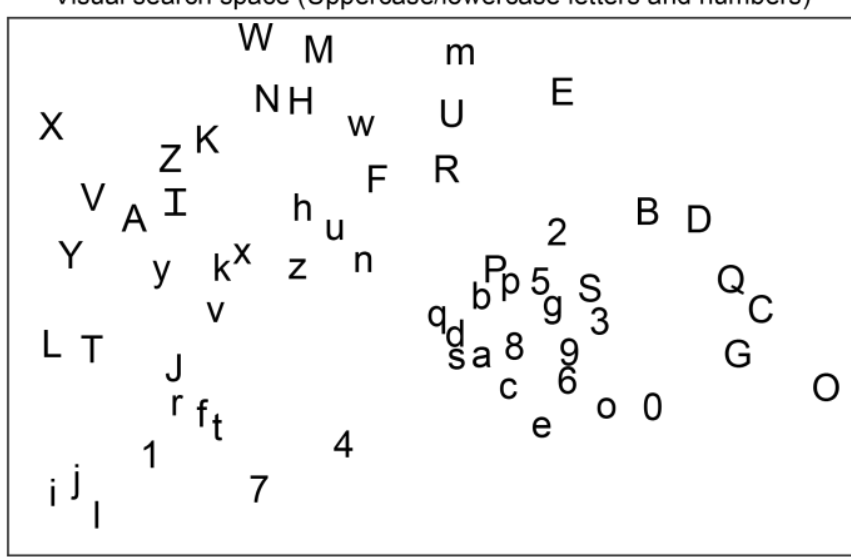

B

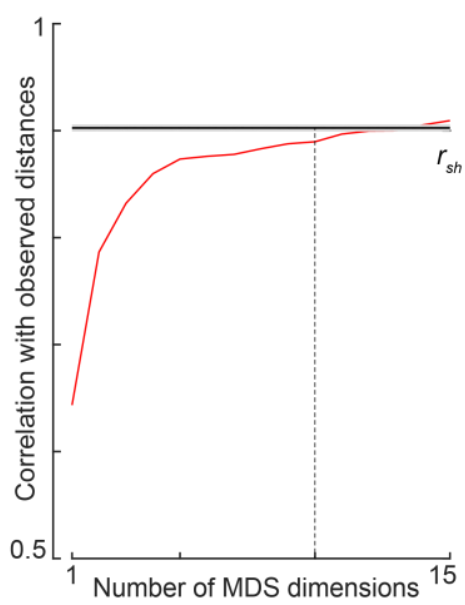

Figure S1. Visual search space for letters and digits

(A) Visual search space for letters (uppercase and lowercase) and digits obtained by multidimensional scaling of observed dissimilarities. Nearby letters represent hard searches. Distances in this $2 \mathrm{D}$ plot are highly correlated with the observed distances $(r=0.79, p<0.00005)$.

(B) Correlation between observed distances and MDS embedding as a function of number of MDS dimensions. The horizontal line represents the split-half correlation with error bars representing s.d calculated across 100 random splits.

\section{Can letter dissimilarity be predicted using low-level visual features?}

To investigate whether single letter dissimilarity can be predicted using lowlevel visual features, we attempted to predict letter dissimilarities using two models. In the first model, which we call the pixel model, we calculated the dissimilarity between letters to be the absolute difference in pixel intensities between the images of the two 
57 letters. This pixel-based model showed a significant correlation $(r=0.50, p<0.00005)$ but was far from the reliability of the data itself $\left(r_{s h}=0.90\right.$; Figure S1B). In the second model, we calculated the dissimilarity between two letters as the vector distance between the responses evoked by a population of simulated V1 neurons (Ratan Murty and Arun, 2015). This V1 model also showed a significant correlation $(r=0.44, p<$ 0.00005 ) but again far from the reliability of the data itself). We conclude that single letter dissimilarity can only be partially predicted by low-level visual features.

\section{Is visual search dissimilarity related to subjective dissimilarity?}

In this study, we have used visual search as a natural and objective measure for visual dissimilarity. However previous studies have measured letter dissimilarity either through confusions in letter recognition, or through subjective dissimilarity ratings (Mueller and Weidemann, 2012; Simpson et al., 2013). We have previously shown that subjective dissimilarity for abstract silhouettes is strongly correlated with visual search dissimilarity (Pramod and Arun, 2016). This may not hold for letters since subjects can activate letter representations that are modified through extensive familiarity. To investigate how visual search dissimilarity compares with subjective similarity ratings for letters, we compared search dissimilarities for uppercase letters against two sets of previously reported similarity data. First, we compared visual search dissimilarities with subjective dissimilarity ratings (Simpson et al., 2013). This revealed a significant positive correlation $(r=0.69, p<0.0005)$. Second, we compared visual search dissimilarities with letter confusion data (3). To convert letter confusion response times, which are a measure of similarity, into dissimilarities, we took their reciprocals, and then compared them with visual search dissimilarities. This revealed a significant positive, albeit weaker correlation $(r=0.34, p<0.0005)$. 
bioRxiv preprint doi: https://doi.org/10.1101/653048; this version posted April 16, 2020. The copyright holder for this preprint (which was not certified by peer review) is the author/funder, who has granted bioRxiv a license to display the preprint in perpetuity. It is made available under aCC-BY-NC-ND 4.0 International license.

\section{SECTION A2. UPRIGHT AND INVERTED BIGRAMS AND TRIGRAMS}

It has been observed that readers are more sensitive to letter transpositions for letters of their familiar script. Since discrimination of letter transpositions in the letter model is a direct consequence of asymmetric spatial summation (main text, Figure 3), we predicted that readers should show more asymmetric spatial summation for familiar letters compared to unfamiliar letters. As a strong test of this prediction, we compared visual search performance on upright letters (which are highly familiar) with inverted letters (which are unfamiliar) across two experiments, one on bigrams and the other on trigrams.

The comparison of upright and inverted letter strings is also interesting for a second reason. If reading or familiarity with upright letters led to the formation of specialized detectors for longer strings, then we predict that the letter model (which assumes responses to be driven by single letters only) should yield worse fits for upright compared to inverted letters.

We tested the above two predictions in the following two experiments.

\section{Experiment 3: Upright vs inverted bigrams}

Methods. A total of 8 subjects ( 6 males, aged $24 \pm 1.5$ years) participated in this experiment. Six uppercase letters: A, L, N, R, S, and T were combined in all pairs to form a total of 36 stimuli. These uppercase letters were chosen because their images change when inverted (as opposed to letters like $\mathrm{H}$ that are unaffected by inversion), and were chosen to maximize the occurrence of frequent bigrams. The same stimuli were inverted to create another set of 36 stimuli. Stimuli subtended $\sim 4^{\circ}$ along the longer dimension. Subjects performed all possible searches among the upright letters $\left({ }^{36} \mathrm{C}_{2}=630\right.$ searches $)$ with two repetitions and likewise for inverted letters. All trials were interleaved. All other details were exactly as in Experiment 2.

\section{Results}

We observed interesting differences in search difficulty depending on the nature of the bigrams. This pattern is illustrated in Figure S2A-B. When the target and distractors consisted of repeated letters (e.g. TT among AA in Figure S2A), search is equally easy when the array is upright or inverted. In contrast if the target and distractors are transposed versions of each other (e.g. TA among AT in Figure S2B), search is easier in the upright array compared to when it is inverted.

To confirm that this effect is present across all such pairs, we compared observed response times for these two types of searches between upright and inverted conditions (Figure S2C). Response times for the AA-BB searches were comparable for upright and inverted conditions (mean $\pm \mathrm{sd}$ of RT: $0.66 \pm 0.09 \mathrm{~s}$ for upright, $0.67 \pm 0.1 \mathrm{~s}$ for inverted). To assess the statistical significance of this difference, we performed an ANOVA with subject (8 levels), bigram (15 pairs) and orientation (upright vs inverted) as factors. We observed no significant difference in the response times between upright and inverted conditions for AA-BB searches $(p=$ 0.65 for main effect of orientation; $p<0.00005$ for subject and bigram factors, $p>0.05$ for all interactions).

Next we compared transposed letter (AB-BA) searches. Here, subjects were clearly faster on the upright searches compared to inverted searches (mean \pm sd of RT: $1.58 \pm 0.25 \mathrm{~s}$ for upright, $3.12 \pm 0.76 \mathrm{~s}$ for inverted). This difference was statistically significant $(p<0.00005$ for main effect of orientation; $p<0.0005$ for subject and $p<$ 
bioRxiv preprint doi: https://doi.org/10.1101/653048; this version posted April 16, 2020. The copyright holder for this preprint (which was not certified by peer review) is the author/funder, who has granted bioRxiv a license to display the preprint in perpetuity. It is made available under aCC-BY-NC-ND 4.0 International license.

.05 for bigram factors, $\mathrm{p}<0.05$ for interactions between pairs and orientation. Other interaction effects were not significant).

To compare bigram dissimilarity between upright and inverted bigrams, we plotted one against the other. This revealed a highly significant correlation $(r=0.80, p$ $<0.00005$; Figure S2D). Here too it can be seen that the transposed letter searches are clearly faster when they are upright whereas the repeated letter searches show no such difference.

Thus, inversion slows down transposed letter searches but not repeated letter searches.

\section{Explaining upright and inverted bigram dissimilarity using the letter model}

We fit the letter model to both upright and inverted bigram searches using a total of 10 neurons with single letter responses derived from Experiment 1 . The letter model yielded excellent fits on both upright and inverted bigrams. In both cases, the model fits approached the data consistency (Figure S2E), implying that the model explained nearly all the explainable variance in the data.

To compare these model fits for upright vs inverted statistically, we performed a bootstrap analysis. Each time, we selected subjects with replacement and fit the letter model to the average dissimilarity computed for this random pool of subjects. Each time we calculated a normalized correlation measure that takes into account the difference in data reliability between upright and inverted trigram searches. This normalized correlation is simply the model correlation divided by the data consistency. To assess statistical significance, we calculated the fraction of times the normalized correlation in the upright samples was larger than the inverted samples. This analysis revealed significant difference in model performance between upright and inverted searches, but in the opposite direction (average model correlation: $r=0.92$ for upright, 0.9 for inverted; fraction of upright < inverted normalized model correlation: $p=0$ ). Thus, upright searches are more predictable than inverted searches using the letter model.

Next we asked whether the letter model can explain the intriguing observation that inversion affects transposed letter searches but not repeated letter searches. This is easy to explain in the letter model: The response to repeated letter bigrams such as $A A$ is unaltered (Figure $3 B$ ), and therefore the dissimilarity between $A A$ and TT is unaffected by the asymmetry in spatial summation. By contrast, the dissimilarity between transposed letter pairs like AT \& TA is directly driven by the asymmetry in spatial summation. We also note that the search TT among AA is much easier than the search for TA among AT. This is also explained by the letter model by the fact that the response to repeated letters is the same as the response to individual letters, leaving their discrimination unaltered. By contrast transposed letters are much more similar since their neural responses are much closer (Figure 3B).

To be sure that letter model predictions show the same pattern, we plotted the average response time predicted by the letter model for repeated letter (AA-BB) and transposed letter (AB-BA) searches. To assess the statistical significance, we performed a sign-rank test on the predicted RT. The letter model predictions were exactly as expected (Figure S2F).

Next we analysed the model parameters in the letter model to ascertain whether the spatial summation in the neurons was indeed different for upright and inverted bigrams. To quantify the degree of asymmetry, we calculated for each neuron a spatial modulation index of the form $\mathrm{Ml}=\mathrm{abs}(\mathrm{w} 1-\mathrm{w} 2) /(\mathrm{w} 1+\mathrm{w} 2)$ where $\mathrm{w} 1$ and $\mathrm{w} 2$ are the estimated weights for each letter in the bigram. To avoid unnaturally large modulation 
indices, w1 and w2 values smaller than 0.01 were set to 0.01 . The spatial modulation index for all 10 neurons for upright and inverted bigrams is shown in Figure S2G. It can be seen that the modulation index is larger in most cases for the upright bigrams. This difference was statistically significant, as assessed using a sign-rank test on the spatial modulation indices (Figure $\mathrm{S} 2 \mathrm{H}$ ).

A

B
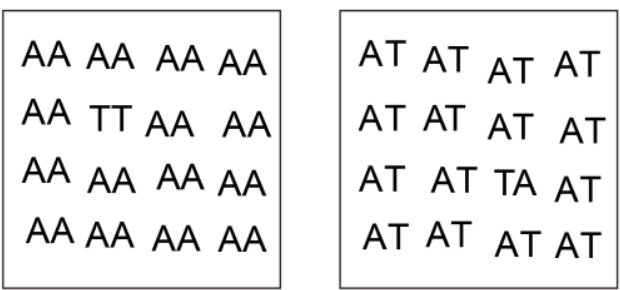

C
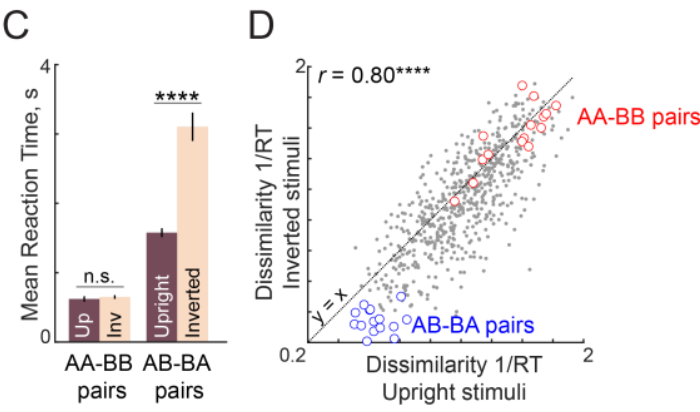

E
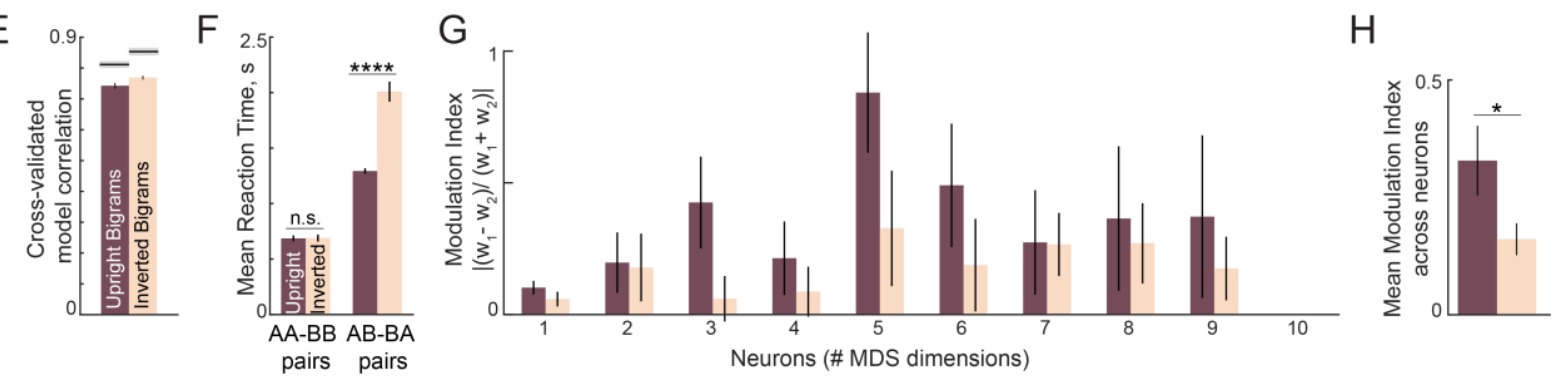

Figure S2. Letter model fits for upright and inverted bigrams

(A) Example oddball search array for a repeated letter target (TT) among identical repeated-letter distractors $(A A)$. It can be seen that inverting this search array does not affect search difficulty.

(B) Example oddball search array for transposed letters (TA among AT). It can be seen by inverting this search array makes the search substantially more difficult.

(C) Average search times in the oddball search task for repeated-letter searches (AA-BB) and transposed letter (AB-BA) searches. Error bars represent s.e.m calculated across subjects. Asterisks represent statistical significance $\left({ }^{* * *}\right.$ is $p$ $<0.00005$ ), as obtained using an ANOVA on the response times with subject, bigram and orientation as factors (see text).

(D) Dissimilarity of inverted bigram pairs plotted against the dissimilarity of upright bigram pairs. Correlation is shown at the top left. Asterisks indicate statistical significance of the correlations $\left({ }^{* * *}\right.$ is $\left.p<0.00005\right)$.

(E) Cross-validated model correlation of the letter model for upright bigrams and inverted bigrams. Shaded gray bars represent the upper bound achievable in each case given the consistency of the data, calculated using the split-half correlation $r_{\text {sh }}$.

(F) Predicted RT from the letter model for repeated letter pairs and transposed letter pairs. Asterisks denote statistical significance as obtained using a signrank test on the predicted RTs between upright and inverted conditions.

(G) Spatial modulation index for each neuron in the letter model for upright and inverted bigrams. 
bioRxiv preprint doi: https://doi.org/10.1101/653048; this version posted April 16, 2020. The copyright holder for this preprint (which was not certified by peer review) is the author/funder, who has granted bioRxiv a license to display the preprint in perpetuity. It is made available under aCC-BY-NC-ND 4.0 International license.

(H) Average spatial modulation index for upright and inverted bigrams. Asterisks represent statistical significance $\left({ }^{*}\right.$ is $p<0.05$ ) obtained using a sign-rank test on the spatial modulation index across the 10 neurons.

\section{Experiment S1: Upright and inverted trigrams}

Here, we asked whether the above results would extend to trigrams. We tested two predictions. First, we predicted greater spatial modulation for upright compared to inverted trigrams, on the premise that better discrimination of trigram transpositions should be driven by asymmetric spatial summation. Second, if repeated viewing of a trigram or word led to the formation of specialized trigram detectors, then the letter model (which is based only on knowledge of single letters) should produce larger errors compared to other trigrams. We tested this prediction by comparing model fits for searches involving frequent trigrams and words compared to other searches.

Methods. A total of 9 subjects ( 6 females, aged $24.5 \pm 2.3$ years) participated in the experiment. Six uppercase letters: A, G, N, R, T and Y were combined in all possible 3-letter combination to form a total of 216 stimuli. These letters were chosen to include as many three-letter words as possible. In all, 15 three-letter words could be created using these letters (ANT, ANY, ART, GAG, GAY, NAG, NAY, RAG, RAN, RAT, RAY, TAG, TAN, TAR, and TRY).

Since the total number of possible search pairs is large $\left({ }^{216} \mathrm{C}_{2}=23,220\right.$ pairs $)$, we chose 500 search pairs such that the regression matrix of the part-sum model had full rank i.e. all the model parameters can be estimated reliably using linear regression. These 500 searches consisted of 368 random search pairs, $105\left({ }^{15} \mathrm{C}_{2}\right)$ word-word pairs, $15\left(3 ! \mathrm{C}_{2}\right)$ transposed pairs of nonword comprised of letters $\mathrm{G}, \mathrm{N}$, and R. Further, another set of $15\left({ }^{3 !} \mathrm{C}_{2}\right)$ transposed pairs were created using the word TAR. The search pairs formed using the words TAR, ART and RAT were presented only once (although they were counted as both word-word pairs and transposed pairs in the main analysis).

Subjects performed the same searches using upright and inverted trigrams. Stimuli subtended $\sim 5^{\circ}$ along the longer dimension. All subjects completed 2000 correct trials (500 searches $\times 2$ orientations $\times 2$ repetitions). All other details were identical to Experiment 1.

\section{Results}

An example oddball array in the trigram experiment is shown in Figure S3A. Note that it is no longer meaningful to compare repeated letter trigrams (AAA-BBB) with transposed trigrams $(A B C-B C A)$ because the repeated letter pairs contain two unique letters whereas the transposed trigrams contain three unique letters. Subjects were highly consistent in both upright and inverted searches (split-half correlation between even and odd- subjects: $r=0.76 \& 0.80, p<0.00005)$. Upright and inverted dissimilarities were highly correlated ( $r=0.80, p<0.00005$; Figure S3B), although upright searches had higher dissimilarity compared to inverted searches.

Next we asked whether the letter model can predict dissimilarities between upright trigrams. As before, letter model predictions were highly correlated with the observed data $(r=0.79, p<0.00005$; Figure S3C) and this model fit approached the data consistency itself $\left(r_{\text {data }}=0.88\right)$. Model fits errors were acctually lower for transposed pairs compared to word-word pairs and other pairs (mean \pm sd error: 0.1 \pm 0.08 for word pairs; $0.07 \pm 0.06$ for transposed pairs; $0.11 \pm 0.08$ for other pairs; $p=$ 0.02 , rank-sum test). The letter model was also able to predict dissimilarities between various trigram transpositions $(r=0.69, p<0.00005$; Figure S3C). Thus, trigram 
dissimilarities can be predicted by the letter model regardless of word status or trigram frequency.

We then compared model fits for upright and inverted trigrams. In both cases, the letter model predictions $(r=0.78 \& 0.73$ for upright and inverted) were close to the consistency of the data ( $r_{\text {data }}=0.85 \& 0.78$; Figure S3D). To compare these model fits for upright vs inverted statistically, we performed a bootstrap analysis as before (Experiment 3). This analysis revealed no significant difference in model performance between upright and inverted searches (fraction of upright $<$ inverted normalized model correlation: $p=0.07$ ).

Finally we asked whether the spatial summation weights of the letter model were systematically different between upright and inverted trigrams. Since there are three spatial modulation weights for each neuron, we calculated the spatial modulation index for all possible pairs of weights (Figure S3 E,F,G). The spatial modulation ratio was larger for upright compared to inverted trigrams in two of the three pairs, and this difference attained statistical significance for the first and third letters in the trigram (Figure S3F). We conclude that the spatial modulation is stronger for upright compared to inverted trigrams.

A

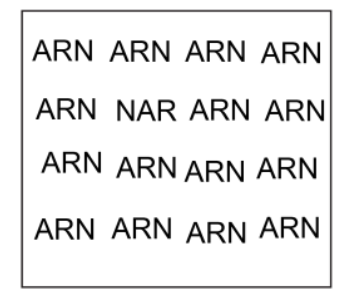

C
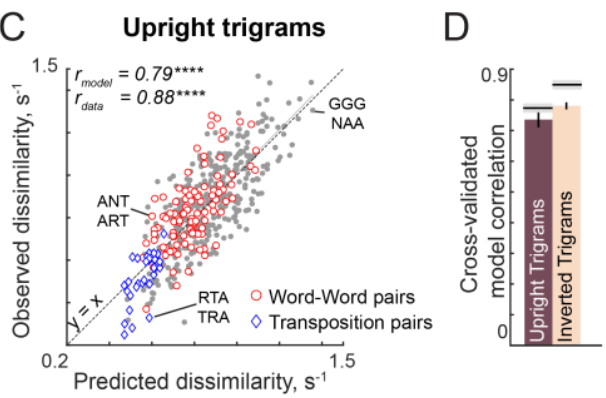

letter mode
B

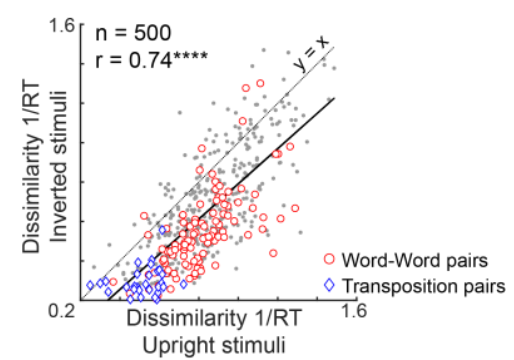

E
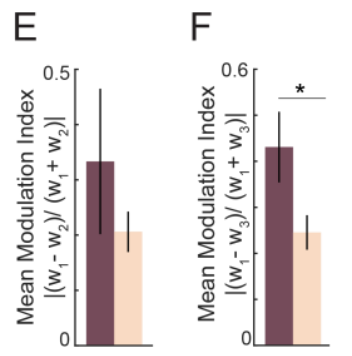

G

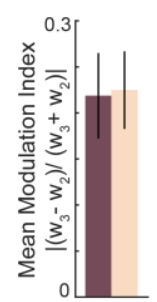

Figure S3. Letter model fits for upright and inverted trigrams

284

285

286

287

288

289

290

291

292

293

294

295

296

(A) Example trigram search array containing letter transpositions, with oddball target (NAR) among distractors (ARN). It can be seen that this search is substantially harder when inverted compared to upright.

(B) Dissimilarity for inverted trigram searches (1/RT) plotted against dissimilarity for upright trigram searches for word-word pairs (red circles, $n=105$ ), transposed letter pairs (blue diamonds, $n=30$ ), and other pairs (gray circles, $n=365$ ).

(C) Observed dissimilarity for upright trigrams plotted against the predicted dissimilarity from the letter model with symbol conventions as in (B).

(D) Cross-validated letter model correlation for upright and inverted trigrams.

(E) Average spatial modulation index (across 10 neurons) for the first and second letters in the trigram.

(F) Same as (E) but for the first and third letters.

(G) Same as (E) but for the second and third letters. 
298

299

300

301

302

303

304

305

306

307

308

309

310

311

312

313

314

315

316

317

318

319

320

321

322

323

324

325

326

327

328

329

330

331

332

333

334

335

336

337

338

339

Here we created compound words by combining two valid words such as FORGET from FOR and GET (Figure S5A). This resulted in some valid words (e.g. FORGET, TEAPOT) and many invalid words (e.g. FORPOT and TEAGET). The full stimulus set is shown in Figure S4.

If valid words are driven by specialized detectors, responses to valid words should be less predictable by the single letter model. We formulated two specific predictions. First, we hypothesize that the dissimilarity between valid words (e.g. FORMAT vs TEAPOT) would yield larger model errors compared to invalid word pairs (e.g. DAYFOR vs ANYMAT). Second, we predicted that the dissimilarity between two invalid compound words (e.g. DAYFOR vs ANYMAT) should be explained better by their constituent trigrams (DAY, FOR, ANY, MAT) rather than by their constituent letters (Figure S5B).

\section{METHODS}

A total of 8 subjects ( 4 female, aged $25 \pm 2.5$ years) participated in the experiment. Twelve 3-letter words were chosen: ANY, FOR, TAR, KEY, SUN, TEA, ONE, MAT, GET, PAD, DAY, POT. Each word was scrambled to obtain twelve 3-letter nonwords containing the same letters. The 12 words were combined to form 36 compound words (Figure S4), such that they appeared equally on the left and right half of the compound words. It can be seen that there are seven valid words, whereas the other compound words are pseudowords that carry no meaning. The compound words measured $6^{\circ}$ along the longer dimension. Subjects completed 1260 correct trials ${ }^{36} \mathrm{C}_{2}$ search pairs $\mathrm{x} 2$ repetitions). Additionally, subjects also performed visual search on 3-letter words $\left(n=132,{ }^{12} C_{2} \times 2\right.$ repetitions) and their jumbled versions $(n=$ 132). Trials timed out after 15 seconds. All other details were identical to Experiment 1.

Subjects were highly accurate on this task (mean \pm std: $98 \pm 1 \%$ ). Outliers in the reaction times were removed using built-in routines in MATLAB (isoutlier function, MATLAB R2018a). This step removed $6.4 \%$ of the response time data.

$\begin{array}{ccccccc} & \text { ANY } & \text { FOR } & \text { TAR } & \text { KEY } & \text { SUN } & \text { TEA } \\ \text { ONE } & \text { ANYONE } & \text { ONEFOR } & \text { ONETAR } & \text { KEYONE } & \text { ONESUN } & \text { TEAONE } \\ \text { MAT } & \text { MATANY } & \text { FORMAT } & \text { MATTAR } & \text { MATKEY } & \text { SUNMAT } & \text { TEAMAT } \\ \text { GET } & \text { GETANY } & \text { FORGET } & \text { TARGET } & \text { KEYGET } & \text { GETSUN } & \text { GETTEA } \\ \text { PAD } & \text { PADANY } & \text { FORPAD } & \text { TARPAD } & \text { KEYPAD } & \text { PADSUN } & \text { PADTEA } \\ \text { DAY } & \text { ANYDAY } & \text { DAYFOR } & \text { TARDAY } & \text { DAYKEY } & \text { SUNDAY } & \text { DAYTEA } \\ \text { POT } & \text { ANYPOT } & \text { POTFOR } & \text { POTTAR } & \text { POTKEY } & \text { SUNPOT } & \text { TEAPOT }\end{array}$

Figure S4. Stimulus set used for Experiment 4 (Compound Words). The left and the right 3 letters words were combined to form a 6 -letter string. The strings that formed compound words are highlighted in red.

\section{RESULTS}

We recruited 8 subjects to perform oddball search involving pairs of trigrams as well as 6-letter strings. In all there were 12 three-letter words which resulted in ${ }^{12} \mathrm{C}_{2}=$ 66 searches and 36 compound 6 -letter strings which resulted in ${ }^{36} \mathrm{C}_{2}=630$ searches. We also included 12 three-letter nonwords created by transposing each three-letter 
bioRxiv preprint doi: https://doi.org/10.1101/653048; this version posted April 16, 2020. The copyright holder for this preprint (which was not certified by peer review) is the author/funder, who has granted bioRxiv a license to display the preprint in perpetuity. It is made available under aCC-BY-NC-ND 4.0 International license.

340 words, resulting in an additional ${ }^{12} \mathrm{C}_{2}=66$ searches. As before, subjects were highly

341

342

343

344

345

346

347

348

349

350

351

352

353

354

355

356

357

358

359

360

361

362

363

364

365

366

367

368

369

370

371

372

373

374

375

376

377

378

379

380

381

382

383

384 consistent in their responses (split-half correlation between odd and even subjects: $r$ $=0.54, p<0.00005$ for 3 -letter words; $r=0.46, p<0.00005$ for 3 -letter nonwords; $r=$ $0.65, p<0.00005$ for 6 -letter words).

We started by using the single letter model as before to predict compound word responses. We took single neuron responses as before from Experiment 1, and took the response of each neuron to a compound word to be a weighted sum of its responses to the individual letters. Using these compound word responses, we calculated the dissimilarity between pairs of compound words, and used nonlinear fitting to obtain the best model parameters. The single letter model yielded excellent fits to the data $(r=0.68, p<0.00005$; Figure S5C). This performance was comparable to the data consistency estimated as before $\left(r_{\text {data }}=0.72\right)$.

Next we asked whether discrimination between compound words can be explained better as a combination of two valid three-letter words, or as a combination of all the constituent six letters. To address this question we constructed a new compositional model based on trigrams, and asked if its performance was better than the single letter model (Figure S5D). The trigram-based letter model used trigram dissimilarity to construct neurons with trigram tuning, and spatial summation over the two trigrams to predict the 6-gram responses. To compare the performance of both models even though they have different numbers of free parameters, we used crossvalidation: we fit both models on half the subjects and tested their performance on the other half. The letter model outperformed the trigram model (Figure S5D). Because both models were trained on half the subjects and tested on the other half, the upper bound on their performance is simply the split-half correlation between the two halves of the data (denoted by $r_{s h}$ ). Indeed the letter model performance was close to this upper bound $\left(r_{s h}=0.56\right.$; Figure S5D), suggesting that it explained nearly all the explainable variance in the data. Finally, the letter model outperformed a widely used model for orthographic distance - the Orthographic Levenshtein Distance (OLD) (Figure S5D). Thus, compound word discrimination can be understood from single letters.

Finally, the letter model fits for word-word pairs and nonword-nonword pairs were not significantly different (Figure S5E). This further validates the absence of local combination detectors (Dehaene et al., 2005) in perception.

\section{Three-letter word and nonword dissimilarities}

To investigate whether the letter model can predict dissimilarities between three-letter words and non-words, we fit a separate letter model with 6 neurons as before to the word and non-word dissimilarities. If frequent viewing of words led to the formation of specialized word detectors, the letter model would show worse model fits compared to nonwords. However, we observed no such pattern: the letter model fits were equivalent for words $(r=0.69, p<0.00005$; Figure S5F) and nonwords $(r=0.57$, $\mathrm{p}<0.00005$; Figure S5F) - and these fits approached the respective data consistencies ( $r_{\text {data }}=0.67$ for words, 0.68 for nonwords). We conclude that three-letter string dissimilarities can be predicted by the letter model regardless of word status. 
A

$$
\begin{array}{llll}
\multicolumn{4}{c}{\text { 3-letter words }} \\
\text { ANY } & \text { ONE } & \text { KEY } & \text { PAD } \\
\text { FOR } & \text { MAT } & \text { SUN } & \text { DAY } \\
\text { TAR } & \text { GET } & \text { TEA } & \text { POT }
\end{array}
$$

\section{compound words}

ANYONE, FORMAT, FORGET,

TARGET, KEYPAD,

SUNDAY, TEAPOT

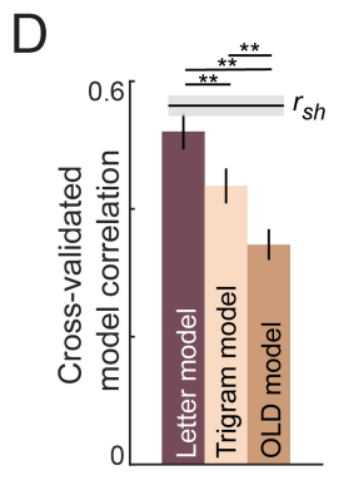

B

$$
\begin{gathered}
\text { Letter model } \\
\text { FORGET }= \\
\mathrm{F}+\mathrm{O}+\mathrm{R}+\mathrm{G}+\mathrm{E}+\mathrm{T}
\end{gathered}
$$
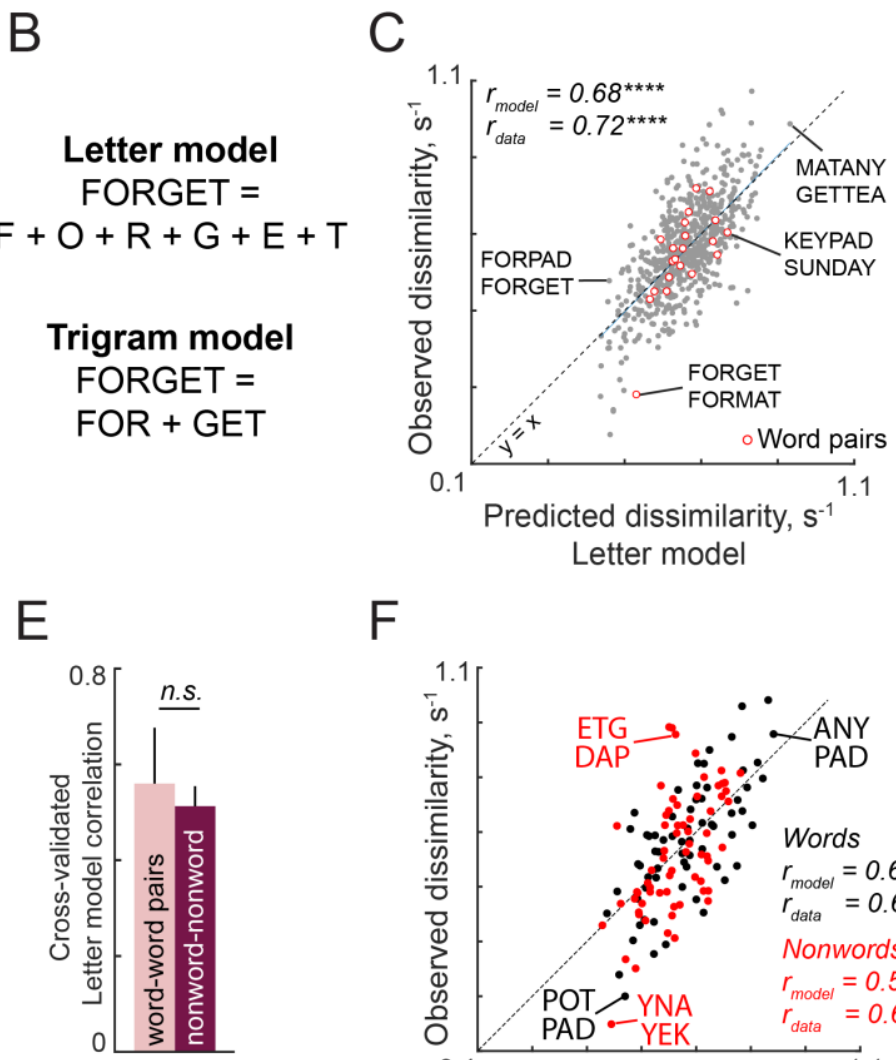

$\mathrm{F}$

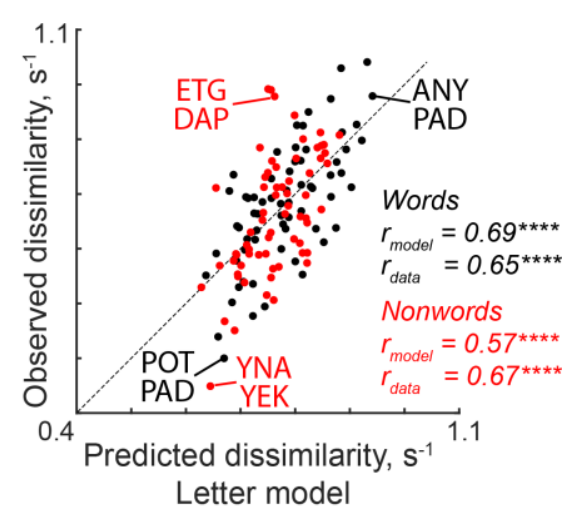

Figure S5. Discrimination of compound words in visual search (Experiment 4).

(A) 3-letter words (top) used to create compound words (bottom).

(B) Illustration of letter and trigram models. In the letter model, the response to a compound word is a weighted sum of responses to the six single letters. In the trigram model, the response to a compound word is a weighted sum of its two trigrams.

(C) Observed dissimilarity for compound words plotted against predicted dissimilarity from the letter model for word pairs ( $r e d)$ and other pairs (gray).

(D) Cross-validated model correlations for the letter model, trigram model and the Orthographic Levenshtein distance (OLD) model. The upper bound on model fits is the split-half correlation $\left(r_{s h}\right)$, shown in black with shaded error bars representing standard deviation across 30 random splits. Horizontal lines above shaded error bar depicts significant difference across different models.

(E) Cross-validated model fits of the letter model for word-words pairs and nonword-nonword pairs.

(F) Observed dissimilarities for 3-letter words (black) and nonwords (red) plotted against letter model predictions. 
bioRxiv preprint doi: https://doi org/10.1101/653048; this version posted April 16, 2020. The copyright holder for this preprint (which was not certified by peer review) is the author/funder, who has granted bioRxiv a license to display the preprint in perpetuity. It is made available under aCC-BY-NC-ND 4.0 International license.

405

406

407

408

409

410

411

412
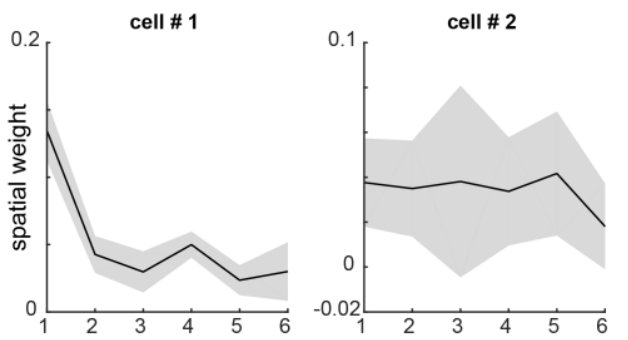

Letter Position

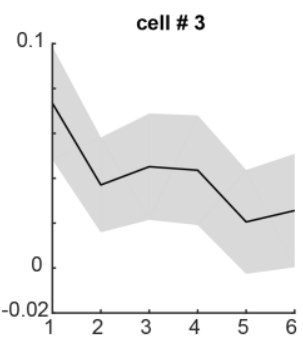

Letter Position

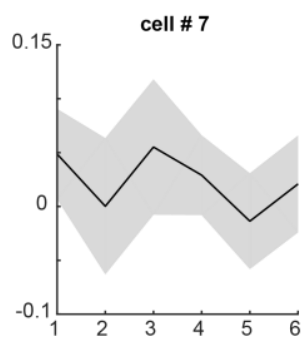

Letter Position

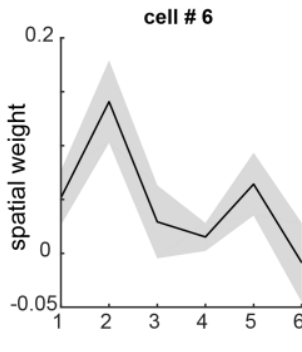

413

414

415

416

417

Figure S6. Spatial summation weights for each neuron. Estimated spatial summation weights (mean \pm std across many random starting points of the nonlinear model fit algorithm) for each neuron in the letter model.
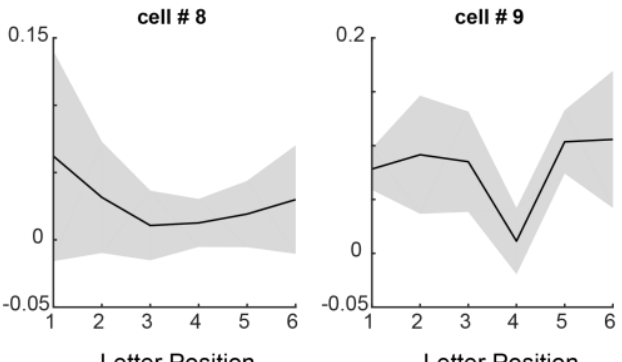

Letter Position
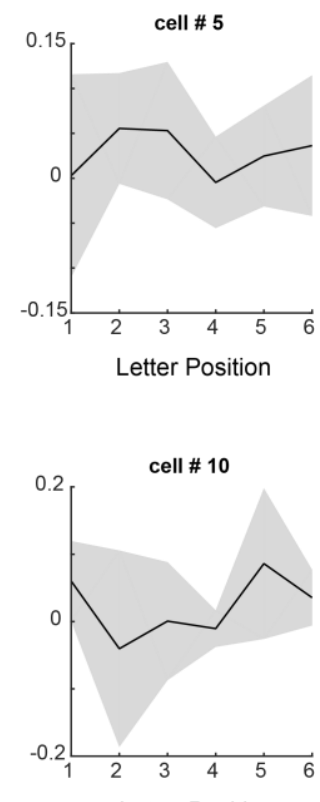

Letter Position 
bioRxiv preprint doi: https://doi.org/10.1101/653048; this version posted April 16, 2020. The copyright holder for this preprint (which was not certified by peer review) is the author/funder, who has granted bioRxiv a license to display the preprint in perpetuity. It is made available under aCC-BY-NC-ND 4.0 International license.

\section{SECTION A4. EXPERIMENTS WITH LONGER STRINGS}

In the main text, we showed that bigram dissimilarity in visual search can be explained using a simple letter model with single letter responses that match perception, and a compositional spatial summation rule that predicts responses to bigrams. Here we asked whether this approach would generalize to longer strings of letters.

To this end, we performed four additional experiments on longer strings. In Experiment S2, we created trigrams with a fixed middle letter and all possible combinations of flanking letters, to create multiple three-letter words. In Experiment S3, subjects performed searches involving 3, 4, 5 and 6-letter searches with uppercase, lowercase and mixed case strings. In Experiments S4 \& S5, we attempted to optimize the search pairs used to estimate model parameters.

\section{METHODS}

Experiment S2: Trigrams with fixed middle letter. A total of 8 subjects ( 5 males, aged $23.9 \pm 1.8$ years) participated in this experiment. Seven uppercase letters: A, E, I, P, $S, T$ and $Y$ were combined (around the stem $R$ i.e. $x R x$ ) in all pairs to form a total of 49 stimuli. These letters were chosen to maximize the occurrence of 3-letter words and psuedowords in the stimulus set. The longer dimension of the stimuli was $\sim 5^{\circ}$. Each subject completed searches corresponding to all possible pairs of stimuli ${ }^{49} \mathrm{C}_{2}=$ 1176) with two trials for each search. All other details were identical to Experiment 2.

Experiment S3: Random string searches. A total of 12 subjects ( 9 female, aged 24.8 \pm 1.64 years) participated in this experiment. All 26 uppercase and lowercase letters were used to create 1800 stimuli, which were organized into 900 stimulus pairs with varying string length. These 900 pairs comprised 3006 -gram uppercase pairs, 1006 gram lowercase pairs, 100 6-gram mixed-case pairs, 100 5-gram uppercase pairs, 50 4-gram uppercase pairs, 50 3-gram uppercase pairs and 200 pairs with uppercase strings of differing lengths (50 pairs each of 6- vs 5-grams, 6- vs 4-grams, 5- vs 4grams, 5- vs 3-grams = 200 pairs total). For each string length, letters were randomly combined to form strings with a constraint that all 26 letters should appear at least once at each location. Each stimulus pair was shown in two searches (with either item as target, and either on the left or right side). The trial timed out at 15 seconds for all searches.

Experiment S4 - Optimized 4-letter searches. In all, 8 subjects (5 females, aged 23.5 \pm 2.3 years) participated in this experiment. To maximize the importance of each spatial location in a 4-letter uppercase string, stimuli were created such that there were at least 75 search pairs with the same letter at either of the corresponding locations. Further, to reliably estimate the model parameters, the randomly chosen letters were arranged to minimize the condition number of the linear regression matrix $X$ of the ISI model described below. In all there were 300 search pairs. The trial timed out after 15 seconds. All other details were similar to Experiment 2.

Experiment S5-Optimized 6-letter searches. A total of 9 subjects (5 males, aged 24.1 \pm 2.2 years) participated in this experiment. We chose 300 search pairs with 6 -letter strings, according to the same criteria as in Experiment S4. All other details were the same as in Experiment S4. 
bioRxiv preprint doi: https://doi.org/10.1101/653048; this version posted April 16, 2020. The copyright holder for this preprint (which was not certified by peer review) is the author/funder, who has granted bioRxiv a license to display the preprint in perpetuity. It is made available under aCC-BY-NC-ND 4.0 International license.

\section{RESULTS}

Cross-validated model fits across all experiments are shown in Figure S7. It can be seen that the letter model fit is close to the split-half consistency of the data. Thus, visual discrimination of longer strings can be explained using a compositional neural code. Below we discuss some experiment-specific findings of interest.

\section{Lowercase and mixed-case strings}

Word shape is thought to play a role in reading lowercase letters, because of the upward deflection (e.g. l, d) and downward deflections (e.g. p, g) of letters which might confer a specific overall shape to a word. To conclusively establish this would require factoring out the contribution of individual letters to word discrimination, as with the letter model. We were therefore particularly interested in whether the letter model would predict the dissimilarity between lowercase and mixed-case strings where word shape might potentially play a role. As can be seen in Figure S7, cross-validated model predictions for lowercase letters were highly correlated with the observed data $(r=$ $0.59, \mathrm{p}<0.00005)$. This correlation approached the upper bound given by the splithalf reliability itself $\left(r_{s h}=0.64\right)$. Likewise, model predictions for mixed-case letters were also highly correlated with the observed data ( $r=0.59, p<0.00005$; Figure S7). However in this case model fits were well below the split-half consistency $\left(r_{s h}=0.72\right)$, suggesting that there is still some systematic unexplained variance in mixed-case strings. This gap in model fit could be simply due to the relatively few mixed-case searches used in this experiment $(n=100)$, or because of unaccounted factors like word shape. Nonetheless, the letter model explains a substantial fraction of variation in both lowercase and mixed case strings, suggesting that it can be used as a powerful baseline to elucidate the contribution of word shape to reading.

\section{Unequal length strings}

The letter model can be used to calculate responses to any string length, provided the spatial summation weights are known. Given the relatively few searches for unequal lengths in our data, we fit the letter model to unequal length strings using 6 neurons. Doing so still raised a fundamental issue: which subset of the 6 spatial summation weights for each neuron should be used to calculate the response to a 4letter string? This requires aligning the 4-letter string to the 6-letter string in some manner.

To address this issue, we evaluated the letter model fit on four possible alignments between longer and shorter strings, and asked whether model predictions were better for any one alignment compared to others. We aligned the smaller length string to either the left, right, centre or edge of the longer string. Model performance for these different variations is shown in Table S1. It can be seen that the model fits are comparable across different choices. However, edge alignment is slightly but not significantly better than other choices. We therefore used edge alignment for all subsequent model predictions. 


\begin{tabular}{|l|c|c|c|c|}
\hline \multirow{2}{*}{ Alignment } & \multicolumn{4}{|c|}{ Letter model correlation } \\
\cline { 2 - 5 } & 6 vs 5 & 6 vs 4 & 5 vs 3 & 4 vs 3 \\
\hline Left: ABCDEF vs EFGHxx & 0.54 & 0.66 & 0.58 & 0.57 \\
\hline Right: ABCDEF vs xxEFGH & 0.51 & 0.66 & 0.57 & 0.58 \\
\hline Centre: ABCDEF vs xEFGHx & - & $\mathbf{0 . 6 8}$ & 0.58 & - \\
\hline Edge: ABCDEF vs EFxxGH & $\mathbf{0 . 5 5}$ & 0.63 & $\mathbf{0 . 6 0}$ & $\mathbf{0 . 5 9}$ \\
\hline
\end{tabular}

512 Table S1: Model fits for various choices of string alignment. In each case we fit

513 the letter model with unknown weights corresponding to the longer length. The 514 alignment is indicated by the position of " $x$ " $s$ in the string. For instance, "Left" alignment means that a 6-letter string ABCDEF is matched to a 4-letter string EFGH by assuming that the response to EFGH is created using the first four weights of spatial summation. Likewise, right alignment means that EFGH is aligned to the right, and therefore its response is created using the last four weights in the 6-letter letter model. The best alignment is highlighted for each column in bold. None of the correlation coefficient differences were statistically significant ( $p>0.05$, Fisher's z-test).

\section{2}

523

524

525

526

527

528

529

530

531

532

533

534

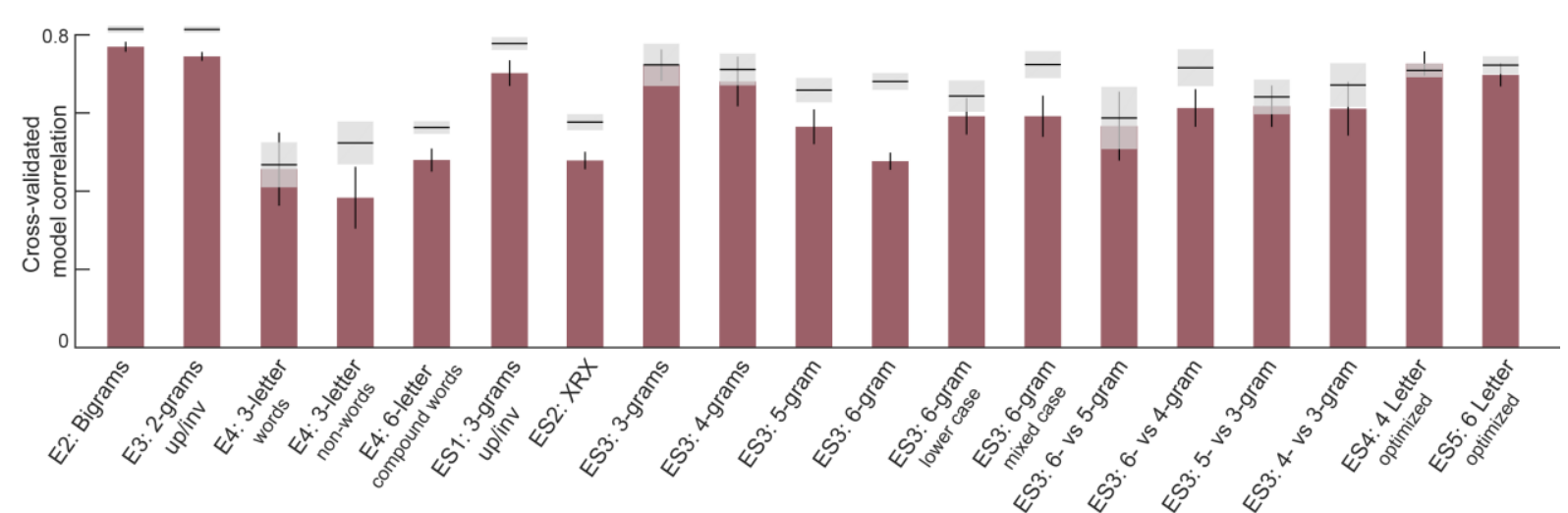

Figure S7. Letter model performance for varying length strings. For each experiment, we obtained a cross-validated measure of model performance using 6 neurons as follows: each time we divided the subjects randomly into two halves, and trained the letter model on one half of the subjects and tested it on the other half. This was repeated for 30 random splits. The correlation between the model predictions and the average dissimilarity from the held-out half of the data was taken to be the model fit. The correlation between the observed dissimilarity between the two random splits of subjects is then the upper bound on model performance (mean \pm std shown as gray shaded bars). 
bioRxiv preprint doi: https://doi.org/10.1101/653048; this version posted April 16, 2020. The copyright holder for this preprint (which was not certified by peer review) is the author/funder, who has granted bioRxiv a license to display the preprint in perpetuity. It is made available under aCC-BY-NC-ND 4.0 International license.

\section{SECTION A5. ESTIMATING LETTER DISSIMILARITY FROM BIGRAMS}

\section{Part-sum model}

The letter model described in the text has many desirable features but requires as input the responses to single letters, which were obtained from searches involving single isolated letters. However, it could be that bigram representations can be understood in terms of component letter responses that are different from the responses of letters seen in isolation. It could also be that letter responses are different at each location.

To address these issues, we developed an alternate model in which bigram dissimilarities can be written in terms of unknown single letter dissimilarities. These single letter dissimilarities can be estimated in the model. In this model, which we call the part-sum model, the dissimilarity between two bigrams $A B \& C D$ is written as the sum of all pairs of part dissimilarities in the two bigrams (Figure S8A). Specifically:

$$
d(A B, C D)=C_{A C}+C_{B D}+X_{A D}+X_{B C}+W_{A B}+W_{C D}+\text { constant }
$$

where $\mathrm{CL}_{\mathrm{AC}}$ is the dissimilarity between letters at Corresponding Left (CL) locations (A $\& \mathrm{C}), \mathrm{CR}_{\mathrm{BD}}$ is the dissimilarity between letters at the Corresponding Right (CR) locations $(B \& D), X_{A D} \& X_{B C}$ are the dissimilarities between letters across locations in the two bigrams $(A \& D, B \& C)$, and $W_{A B} \& W_{C D}$ are the dissimilarities of letters within each bigram.

The part-sum model works because a given letter dissimilarity CLAC will occur in the dissimilarity of many bigram pairs (e.g. in the pair AB-CD and in AE-CF) thereby allowing us to estimate its unique contribution. Since there are 7 parts, there are ${ }^{7} \mathrm{C}_{2}$ $=21$ possible part-pairs of each type (i.e. for $\mathrm{CL}, \mathrm{CR}, \mathrm{X}$ and $\mathrm{W}$ terms), resulting in 21 x 4 = 84 unknown part dissimilarities. Since a given bigram experiment contains all possible ${ }^{49} \mathrm{C}_{2}=1176$ bigram searches, there are many more observations than unknowns. The combined set of bigram dissimilarities can be written in the form of a matrix equation $\mathbf{y}=\mathbf{X b}$ where $\mathbf{y}$ is a $1176 \times 1$ vector of observed bigram dissimilarities, $\mathbf{X}$ is a $1176 \times 85$ matrix containing the number of times $(0,1$ or 2$)$ a given letter-pair of each type (CL, CR, $X \& W$ ) contributes to the overall dissimilarity, and $b$ is a $85 \times 1$ vector of unknown letter dissimilarities of each type (21 each of CL, CR, X \& W and one constant term). The unknown letter dissimilarities of each type was estimated using standard linear regression (regress function, MATLAB).

The part sum model has several advantages over the letter model: (1) It is linear which means that its parameters can be uniquely estimated; (2) it is compositional in that the net dissimilarity between two bigrams is explained using the constituent parts without invoking more complex interactions; (3) it can account for potentially different part relations at each location in the two bigrams. We have previously shown that the part-sum model can explain the dissimilarities between a variety of objects (Pramod and Arun, 2016).

The part sum model yielded excellent fits to the data $(r=0.88, p<0.00005$; Figure S8B) that were close to the reliability of the data $\left(r_{\text {data }}=0.90\right)$. As before, we observed no systematic deviations between model fits for frequent bigrams compared to infrequent bigrams (Figure S8B; average absolute residual error for the top 20 bigram pairs with highest mean bigram frequency: $0.09 \pm 0.1 \mathrm{~s}^{-1}$; for the bottom-20 bigram pairs: $0.11 \pm 0.08 \mathrm{~s}^{-1} ; p=0.42$, rank-sum test). To assess whether the part dissimilarities of each type (CL, CR, $X$ and $W$ ) were related to each other, we plotted each of $C R, X$ and $W$ terms against the $C L$ terms (Figure $S 8 C$ ). The $C R$ and $X$ terms 
585

586

587

588

589

590

591

592

593

594

595

596

597

598

599

600

601

602

603

604

605

606

607

608

609

610

611

612

613

614

615

616

617

618

619

620

621

622

623

624

625

626

627

628

629

630

631

632

were highly positively correlated (Figure S8C), whereas the W terms were negative in sign and negatively correlated (Figure S8C). The negative values of the $\mathrm{W}$ terms means that bigrams with dissimilar letters become less dissimilar, an effect akin to distractor heterogeneity in visual search (Duncan and Humphreys, 1989; Vighneshvel and Arun, 2013). We conclude that the CL, CR, $X$ and $W$ terms in the part-sum model are driven by a common part representation.

To visualize this underlying letter representation, we performed multidimensional scaling on the estimated part dissimilarities of the CL terms. In the resulting plot, nearby letters represent similar letters (Figure S8D). It can be seen that I \& $T, M \& N$ are similar as in the single-letter representation (Figure S1A). These single letter dissimilarities estimated from bigrams using the part-sum model were highly correlated with the single-letter dissimilarities directly observed from visual search with isolated letters (Figure S8D).

We conclude that bigram dissimilarities can be predicted from a common underlying letter representation that is identical to that of single isolated letters.

\section{Equivalence between part-sum and letter model}

Given that the part-sum model and letter model both give equivalent fits to the data, we investigated how they are related. Consider a single neuron whose response to a bigram $\mathrm{AB}$ is given by: $r_{A B}=\alpha r_{A}+r_{B}$, where $r_{A}$ and $r_{B}$ are its responses to $\mathrm{A} \& \mathrm{~B}$, and $\alpha$ is the spatial weight of $A$ relative to $B$. Similarly its response to the bigram $C D$ can be written as $r_{C D}=\alpha r_{C}+r_{D}$. Then the dissimilarity between AB and CD can be written as

$$
\begin{aligned}
& d(A B, C D)^{2} \\
& =\left(r_{A B}-r_{C D}\right)^{2}=\left(\alpha r_{A}+r_{B}-\alpha r_{C}-r_{D}\right)^{2} \\
& =\left(\alpha\left(r_{A}-r_{C}\right)+\left(r_{B}-r_{D}\right)\right)^{2} \\
& =\alpha^{2}\left(r_{A}-r_{C}\right)^{2}+\left(r_{B}-r_{D}\right)^{2}+2 \alpha\left(r_{A}-r_{C}\right)\left(r_{B}-r_{D}\right) \\
& =\alpha^{2}\left(r_{A}-r_{C}\right)^{2}+\left(r_{B}-r_{D}\right)^{2}+2 \alpha\left(r_{A} r_{B}+r_{C} r_{D}-r_{A} r_{D}-r_{B} r_{C}\right) \\
& =\alpha^{2}\left(r_{A}-r_{C}\right)^{2}+\left(r_{B}-r_{D}\right)^{2}+\alpha\left[\left(r_{A}-r_{D}\right)^{2}+\left(r_{B}-r_{C}\right)^{2}-\left(r_{A}-r_{B}\right)^{2}-\left(r_{C}-r_{D}\right)^{2}\right] \\
& =\alpha^{2} d_{A C}^{2}+d_{B D}^{2}+\alpha\left(d_{A D}^{2}+d_{B C}^{2}-d_{A B}^{2}-d_{C D}^{2}\right) \\
& =\alpha^{2} d_{A C}^{2}+d_{B D}^{2}+\alpha\left(d_{A D}^{2}+d_{B C}^{2}\right)-\alpha\left(d_{A B}^{2}+d_{C D}^{2}\right)
\end{aligned}
$$

Thus, the squared dissimilarity between $A B$ \& $C D$ can be written as a weighted sum of squared dissimilarities between parts at corresponding locations (A-C \& B-D), parts at opposite locations (A-D \& B-C) and between parts within each bigram (A-B \& C-D), which is essentially the same as the part-sum model. The same argument extends to multiple neurons because the total bigram dissimilarity will be the sum of bigram dissimilarities across all neurons.

There are however two important differences. First, the part sum model is written in terms of a weighted sum of part dissimilarities, whereas the above equation refers to a weighted sum of squared dissimilarities. However, the squared sum of distances and a weighted sum of distances are highly correlated, so the essential relation will still hold. Second, the letter model predicts that the across-bigram terms $\left(\mathrm{X}_{\mathrm{AD}}, \mathrm{X}_{\mathrm{BC}}\right)$ should be similar in magnitude but opposite in sign to the within-bigram terms $\left(\mathrm{W}_{\mathrm{AB}}, \mathrm{W}_{\mathrm{CD}}\right)$. These weights are similar in magnitude but not exactly equal, as can be seen in Fig S8C. The part-sum model thus allows for greater flexibility in part interactions compared to the letter model. 


\section{Reducing part-sum model complexity (ISI model)}

The observation that a common set of letter dissimilarities drive the part-sum model suggests that the part-sum model can be simplified. We therefore devised a reduced version of the part-sum model - called the Independent Spatial Interaction (ISI) model - in which the CL, CR, X and W terms are scaled versions of the single letter dissimilarities (Figure S8E). Specifically, the dissimilarity between bigrams AB \& CD is:

$$
d(A B, C D)=\alpha_{10} d_{A C}+\alpha_{20} d_{B D}+\alpha_{11}\left(d_{A D}+d_{B C}\right)+\beta_{11}\left(d_{A B}+d_{C D}\right)+c
$$

where $d_{A C}$ is the observed dissimilarity between the left letters $A \& C$ from visual search and $\alpha_{10}$ is an unknown scaling term, $d_{B D}$ is the observed dissimilarity between the right letters $B \& D$, and $\alpha_{20}$ is an unknown scaling term. Likewise, $\alpha_{11}$ is an unknown scaling term for the net dissimilarity $\left(d_{A D}+d_{B C}\right)$ between letters across locations, $\beta_{11}$ is the unknown scaling term for the net dissimilarity $\left(d_{A B}+d_{C D}\right)$ between letters within the two bigrams and $c$ is a constant. Thus, the ISI model has only 5 free parameters: $\alpha_{10}, \alpha_{20}, \alpha_{11}, \beta_{11}$ and $c$. These parameters can be estimated by solving the matrix 650 equation $\mathbf{y}=\mathbf{X b}$ where $\mathbf{y}$ is a $1176 \times 1$ vector of observed bigram dissimilarities, $\mathbf{X}$ is a $1176 \times 5$ matrix containing the net single dissimilarity of each type (CL, CR, X \& W) that contributes to the total dissimilarity, and $\mathbf{b}$ is a $5 \times 1$ vector of unknown weights corresponding to the contribution of each type of dissimilarity (plus a constant).

The performance of the ISI model is summarized in Figure S8F. It can be seen that, despite having only 5 free parameters compared to 85 parameters of the partsum model, the ISI model yields comparable fits to the data (Figure S8F). 


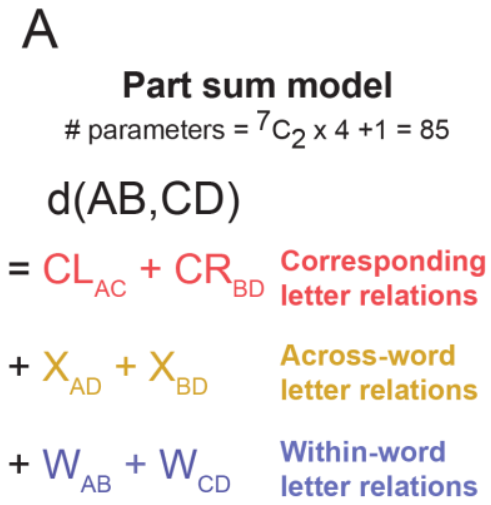

D

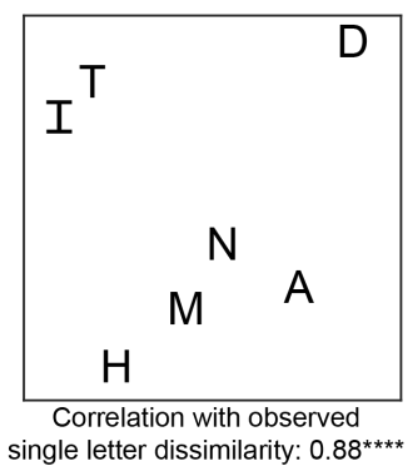

B

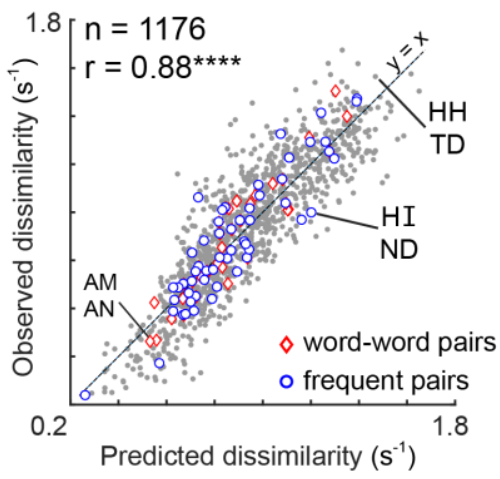

E

$$
\begin{gathered}
\text { Independent Spatial } \\
\text { Interaction model (ISI) } \\
\text { \# parameters }=2^{2}+1=5
\end{gathered}
$$

$$
\mathrm{d}(\mathrm{AB}, \mathrm{CD})=
$$$$
\alpha_{10} d(A, C)+\alpha_{20} d(B, D)
$$$$
+\alpha_{11}[d(A, D)+d(B, C)]
$$$$
+\beta_{11}[\mathrm{~d}(\mathrm{~A}, \mathrm{~B})+\mathrm{d}(\mathrm{C}, \mathrm{D})]
$$

C

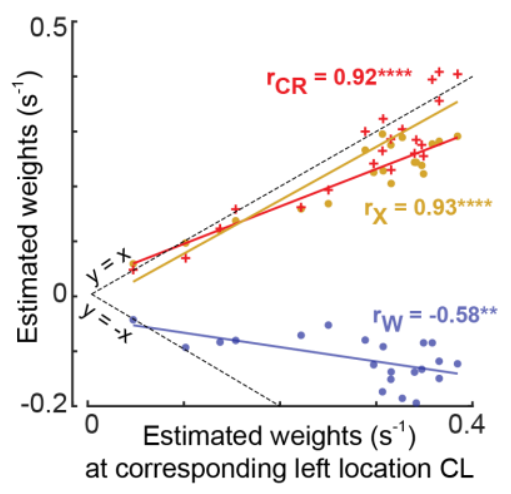

F

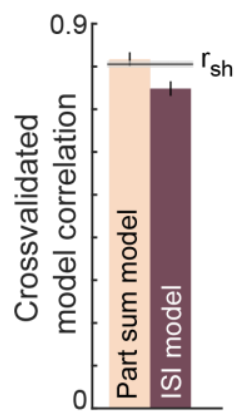

659

660

661

662

663

664

665

666

667

668

669

670

671

672

673

674

675

676

677

678

679

680

681

682

683

684
Figure S8. Predicting bigram dissimilarity using part-sum model

(A) Schematic of the part sum model. According to this model, the dissimilarity (1/RT) between bigrams 'AB' and ' $C D$ ' is written as a linear sum of dissimilarities of its corresponding part terms ( $A C$ and $B D$, shown in red), across part terms ( $A D$ and $B C$, shown in yellow), and within part terms ( $A B$ and $C D$, shown in blue).

(B) Correlation between the observed and predicted dissimilarities (1/seconds). Each point represents one search pair $\left(n={ }^{49} \mathrm{C}_{2}=1176\right)$. Word-word pairs are highlighted using red diamonds, and frequent bigram pairs are highlighted using blue circles. Dotted lines represent unity slope line.

(C) Correlation between the estimated weights at corresponding location left with estimated weights at 1) corresponding location right (red), 2) across location (yellow), and 3) within location (blue). Each point represents one letter pair $\left(n={ }^{7} C_{2}\right.$ $=21$ ). Dotted lines represent positive and negative unity slope line.

(D) Perceptual space of the single letter dissimilarities, that are the model coefficients of part terms at left corresponding location

(E) Schematic of the Independent Spatial Interaction model. In this model, we use the observed letter-pair dissimilarities and only estimate the weights of these letter-pair dissimilarities across different locations.

(F) Comparing part-sum and ISI model fits. Bar plots represents mean correlation coefficient between the observed and predicted dissimilarities. Error bars represent one standard deviation across 30 splits. Black horizontal line represents mean split-half correlation $\left(r_{\text {sh }}\right)$ and the shaded error bar represents one standard deviation around the mean. $\left({ }^{* * * *}, \mathrm{p}<0.00005,{ }^{* *}, \mathrm{p}<0.005\right)$. 
bioRxiv preprint doi: https://doi.org/10.1101/653048; this version posted April 16, 2020. The copyright holder for this preprint (which was not certified by peer review) is the author/funder, who has granted bioRxiv a license to display the preprint in perpetuity. It is made available under aCC-BY-NC-ND 4.0 International license.

685

686

687

688

689

690

691

692

693

694

695

696

697

698

699

700

701

702

703

704

705

706

707

708

709

710

711

712

713

\section{ISI model performance across all experiments}

Next we asked whether the ISI model can be generalized to explain dissimilarities between longer strings. Consider two n-letter strings $u_{1} u_{2} u_{3} u_{4} \ldots u_{n}$ and $v_{1} v_{2} v_{3} v_{4} \ldots v_{n}$. The net dissimilarity between the two strings can be written as:

$$
d\left(u_{1} u_{2} \ldots u_{n}, v_{1} v_{2} \ldots v_{n}\right)=\sum_{i=0}^{n} \sum_{k=0}^{n-i} \alpha_{i k}\left(d\left(u_{i}, v_{i+k}\right)+d\left(v_{i}, u_{i+k}\right)\right)-\sum_{i=0}^{n} \sum_{k=1}^{n-i} \beta_{i k}\left(d\left(u_{i}, u_{i+k}\right)+d\left(v_{i}, v_{i+k}\right)\right)+c
$$

where $\alpha_{i k}$ are the unknown weights corresponding to pairs of letters across the two ngrams separated by "k" positions starting from 0 , and $\beta_{i k}$ are the unknown weights corresponding to pairs of letters separated by " $k$ " positions within the two n-grams. Written in this manner, the total number of unknowns in the $n$-gram ISI model is $n^{2}+1$, which can be estimated using standard linear regression as before. For instance, for the 6 -gram ISI model, there are $6^{2}+1=37$ free parameters.

In this manner, we fit the ISI model to all experiments. The resulting crossvalidated model fits are shown together with the letter model in Figure S9. It can be seen that the ISI model performance is comparable to that of the letter model across all experiments.

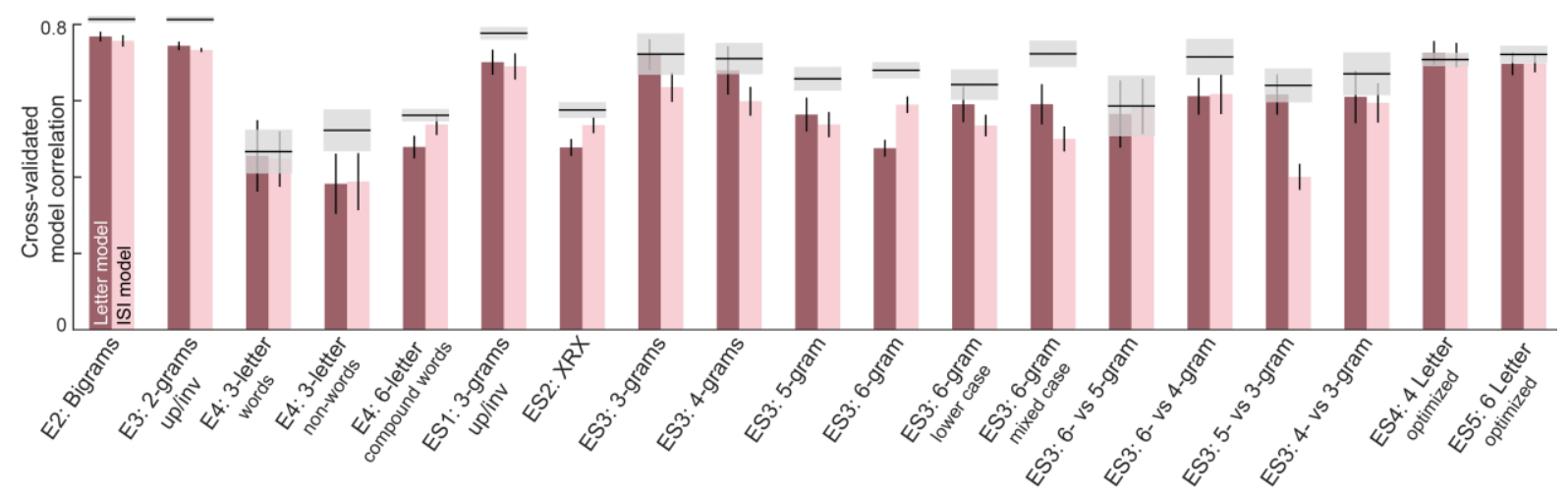

Figure S9. ISI \& letter model performance across all experiments

For each experiment, we obtained a cross-validated measure of both neural and ISI model performance as follows: each time we divided the subjects randomly into two halves, and trained the letter model on one half of the subjects and tested it on the other half. This was repeated for 30 random splits. The correlation between the model predictions and the average dissimilarity from the held-out half of the data was taken to be the model fit. The correlation between the observed dissimilarity between the two random splits of subjects is then the upper bound on model performance (mean \pm std shown as gray shaded bars). 
bioRxiv preprint doi: https://doi.org/10.1101/653048; this version posted April 16, 2020. The copyright holder for this preprint (which was not certified by peer review) is the author/funder, who has granted bioRxiv a license to display the preprint in perpetuity. It is made available under aCC-BY-NC-ND 4.0 International license.

\section{Reducing the complexity of the ISI model}

According to the ISI model, the net dissimilarity between two n-grams can be written as a weighted sum of dissimilarities between letter pairs that are varying distances apart. We wondered if the ISI model can be simplified further if there is a systematic pattern whereby these weight corresponding to a given letter pair varies systematically with letter position and distance between the letters.

To assess this possibility, we plotted model coefficients of the ISI model estimated from Experiment S3 along two dimensions. First, we asked if the contribution of letter pairs at corresponding locations in the two n-grams varies with letter position. For varying string lengths (3-, 4-, 5- and 6-letter strings) we observed a characteristic U-shaped function whereby the edge letters contribute more to the net dissimilarity compared to the middle letters (Figure S10A). Second, we asked if model weights decrease systematically with inter-letter distance. This was indeed the case regardless of the starting letter in the pair (Figure S10B). Finally, we note that across and within part terms are roughly equal in magnitude but opposite in sign (Figure S8C).

The above pattern of weights in the ISI model suggest that we can make two simplifying assumptions. First, the weight of the starting letter is a U-shaped function when the inter-letter distance is zero $\left(\alpha_{i 0}\right)$. Second, weights decrease exponentially thereafter with increasing inter-letter distance. Specifically:

$$
\begin{gathered}
\alpha_{i 0}=a i^{2}+b i+c \text { for } i=1,2, \ldots n \\
\alpha_{i k}=\alpha_{i 0} e^{-k / \tau} \text { for } k \geq 1 \\
\beta_{i k}=-\alpha_{i k} \text { for } k \geq 1
\end{gathered}
$$

where $a, b, c$ and $\tau$ are the free parameters in this model. This simplified model, which we call the Spatial Interaction Decay (SID) model has only 5 parameters and can be used to predict the dissimilarities between strings of arbitrary length. The model parameters are obtained using nonlinear gradient descent methods (nlinfit function, MATLAB).

To illustrate the performance of the SID model in comparison to the ISI model, we fit the model to 6-letter compound words (Experiment 4). To compare the two models, we plotted the ISI model terms directly estimated from the search data against the ISI model terms predicted from the SID model. This yielded a strong positive correlation (Figure S10C). The SID model also yielded excellent fits to the data (Figure S10D), and both models yielded comparable fits (Figure S10E).

To evaluate this pattern across all experiments, we fit both SID and ISI models to all experiments. Here too we obtained qualitatively similar fits for the two models (Figure S11). To confirm whether the SID model trained on one experiment can capture the variations in another, we trained the SID model on data from Experiment S5 and evaluated it on all other experiments. This too yielded largely similar but smaller predictions (Figure S11). This decrease in model fit suggests that model parameters are somewhat dependent on the search pairs chosen.

We conclude that dissimilarities between arbitrary letter strings can be predicted using highly simplified models that operate on single letter dissimilarities and simple compositional rules. 
A

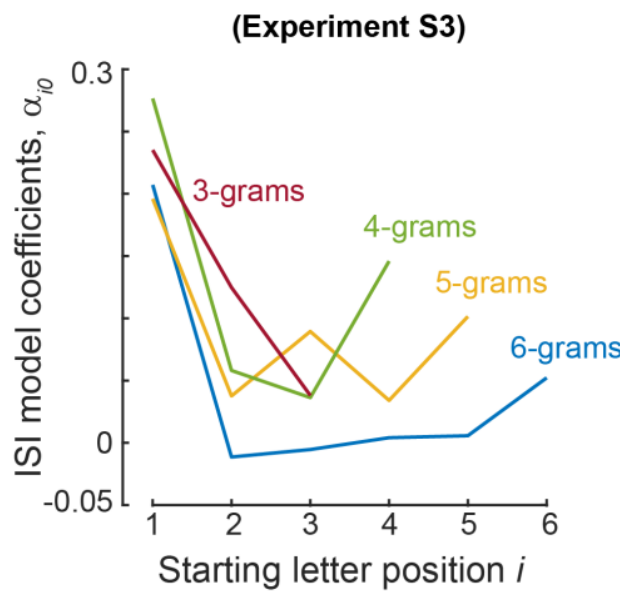

B

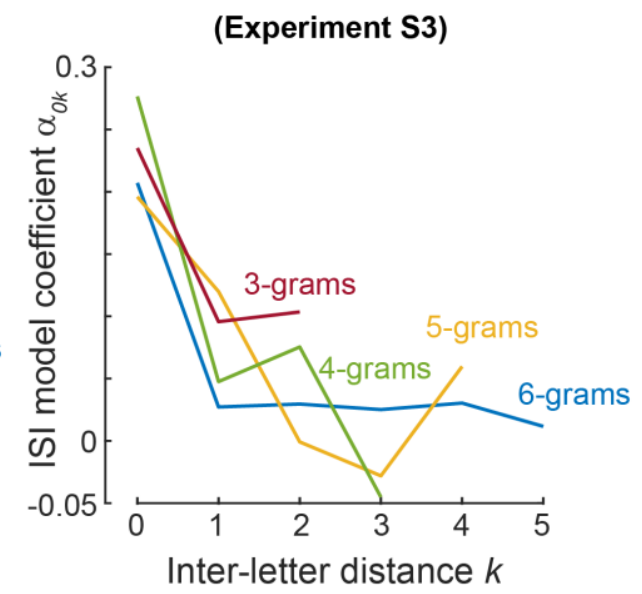

$E$
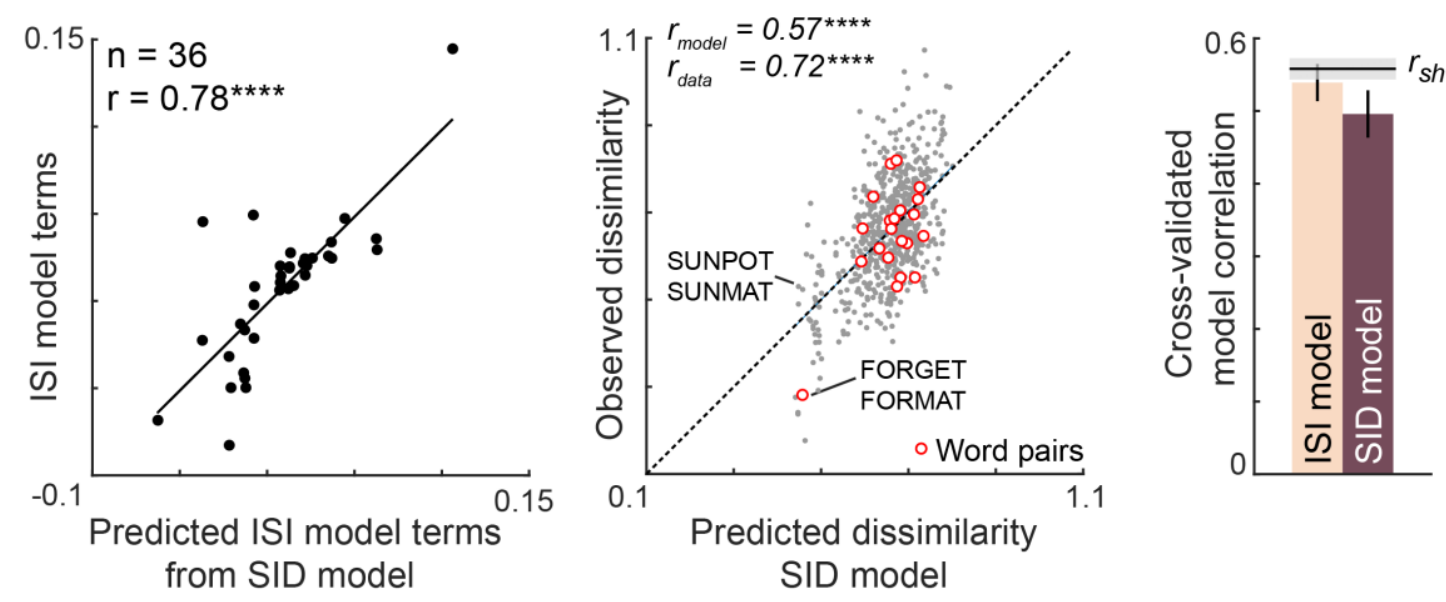

\section{Figure S10. Reducing the ISI model}

(A) ISI model coefficients $\alpha_{i 0}$ as a function of starting letter position $i$, for Experiment S3, for varying string lengths.

(B) ISI model coefficients $\alpha_{1 k}$ as a function of inter-letter distance $k$ for Experiment S3, for varying string lengths.

(C) ISI model coefficients (both $\alpha_{i k}$ and $\beta_{i k}$ ) plotted against the predicted ISI model coefficients from the SID model. Both models are fitted to data from Experiment 4 (compound words).

(D) Observed dissimilarity in Experiment 4 plotted against predicted dissimilarity from the SID model. 


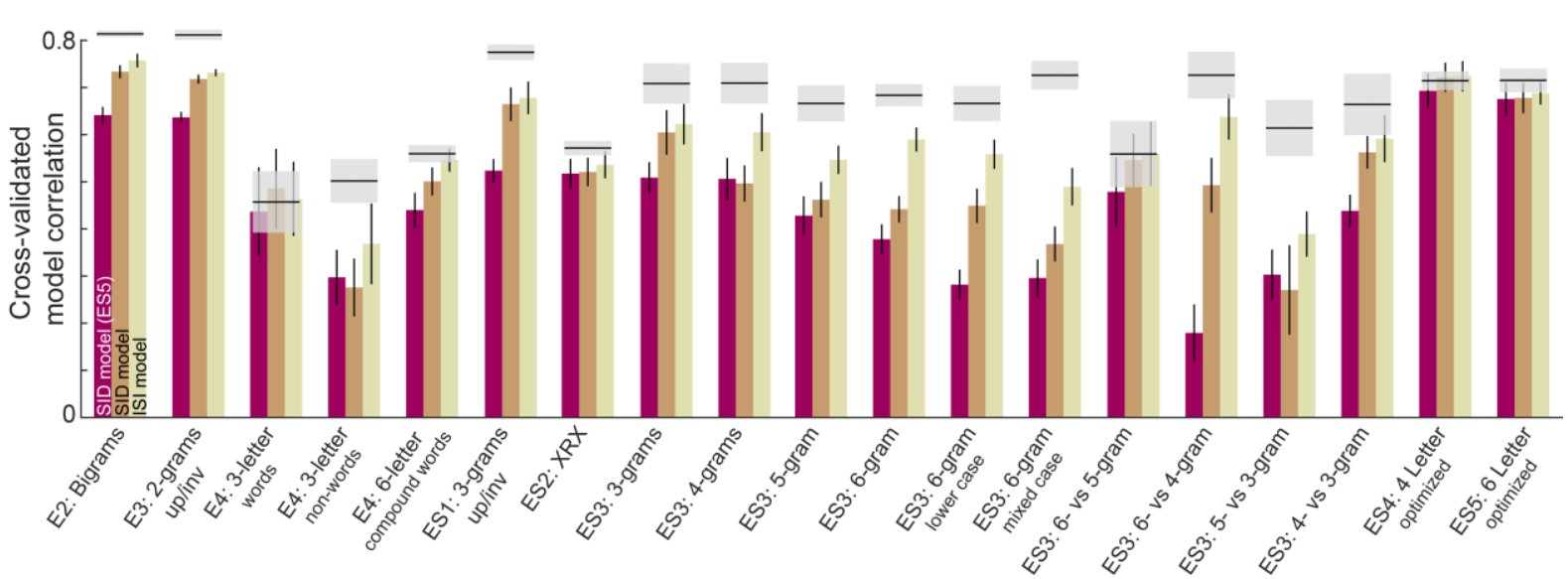

Figure S11. ISI and SID model fits across all experiments. Cross-validated model fits for the ISI and SID models across all experiments. In each case the SID and ISI models were fit on a randomly chosen half of the subjects and tested on the other half. The SID (ES5) bars refer to the SID model trained on Experiment S5 and tested on data from a randomly chosen half of subjects in each experiment. 


\section{Comparing upright and inverted bigrams using part-sum model}

The results in Section A2 were based on fitting the letter model to upright and inverted bigrams but assuming a fixed set of single letter responses derived from uppercase letters. The fact that the letter model yielded excellent fits to both upright and inverted bigrams validates this assumption. Nonetheless, we wondered whether differences between upright and inverted bigram searches can be explained solely by different letter representations or by differences in letter interactions.

To investigate this possibility, we fit the part-sum model to upright and inverted bigram searches (Figure S12A). The part-sum model also yielded equivalent fits to both upright and inverted searches (Figure S12B). If model predictions were similar, we reasoned that the difference between upright and inverted searches must be explained by differences in model parameters. To this end, we compared the estimated letter dissimilarities of each type (CL, CR, $X$ and $W$ ) in the upright and inverted searches (Figure S12C). Model terms were comparable in magnitude for the $\mathrm{CL}$ terms, but were systematically weaker for both $\mathrm{CR}, \mathrm{X}$ and $\mathrm{W}$ terms for inverted compared to upright searches (Figure S12C). However in all cases, the recovered letter dissimilarities were correlated between upright and inverted conditions (correlation between upright and inverted model terms: $r=0.93,0.91,0.97 \& 0.87$ for $\mathrm{CL}, \mathrm{CR}, \mathrm{X} \& \mathrm{~W}$ terms; all correlations $\mathrm{p}<0.00005)$.

799

A

Part sum model

\# parameters $={ }^{7} \mathrm{C}_{2} \times 4+1=85$

$\mathrm{d}(\mathrm{AB}, \mathrm{CD})$

$=C L_{A C}+C R_{B D} \begin{aligned} & \text { Corresponding } \\ & \text { letter relations }\end{aligned}$

$+X_{\mathrm{AD}}+\mathrm{X}_{\mathrm{BD}} \quad \begin{aligned} & \text { Across-word } \\ & \text { letter relations }\end{aligned}$

$+\mathrm{W}_{\mathrm{AB}}+\mathrm{W}_{\mathrm{CD}} \quad \begin{aligned} & \text { Within-word } \\ & \text { letter relations }\end{aligned}$

+ constant

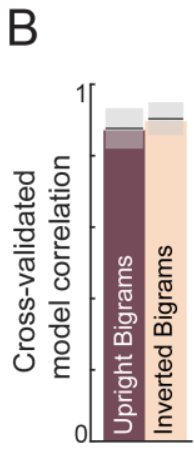

800

801

802

803

804

805

806

807

808

809

810

811

812

\section{Figure S12. Part-sum model fits for upright and inverted bigrams}

(A) Schematic of the part-sum model, in which the net dissimilarity between two bigrams is given as a linear sum of letter dissimilarities at corresponding locations (CL \& CR), across-bigrams $(X)$ and within-bigrams (W).

(B) Cross-validated model correlation of the part sum model for upright and inverted bigrams.

(C) Average model coefficients (mean \pm sem) of each type for upright and inverted bigrams. Asterisks denote statistical significance $\left(^{* * \star}\right.$ is $\left.p<0.00005\right)$ obtained on a sign-rank test comparing 15 letter dissimilarities between upright and inverted conditions). 


\section{Comparing upright and inverted trigrams using part-sum model}

814

815

816

817

818

819

820

821

822

823

824

825

826

827

828

829

830

831

832

833

834

835

836

837

838

839

840

841

842

843

844

845

846

847

848

849
The part sum model applied to trigrams is depicted in Figure S13A. In this model, the net dissimilarity between two trigrams can be written as a sum of single letter dissimilarities at every possible pair of locations. These locations are grouped as corresponding letters at left (C1), middle (C2) and right (C3) locations, letters across trigrams that are one letter apart starting from the left letter (XN1) or the middle letter (XN2), letters across trigrams that are two letters apart (XF), letters within each trigram that are one letter apart starting from the left letter (WN1) or middle letter (WN2), and letters within each trigram that are two letters apart (WF). Thus the full part-sum model has 9 groups of letter dissimilarities (C1, C2, C3, XN1, XN2, XF, WN1, WN2, WF) each having ${ }^{6} \mathrm{C}_{2}=15$ unknown single letter dissimilarities. Together with a constant term, this part-sum model has $9 \times 15+1=136$ free parameters. Since we have 500 searches each for upright and inverted trigrams, the part-sum model can be fit to this data to estimate these free parameters using standard linear regression.

Cross-validated model fits for the part-sum model are shown in Figure S13B. It can be seen that the part-sum model explains nearly all the explainable variance in the data for both upright and inverted trigrams (Figure S13B). This in turn means that differences between upright and inverted trigrams can be explained using differences in model parameters. This was indeed the case: on plotting the strength of model terms of each type it was clear that 7 of the 9 types of model terms $(\mathrm{C} 1, \mathrm{C} 2, \mathrm{C} 3, \mathrm{XN2}, \mathrm{XF}$, WN2, WF) were systematically larger for upright trigrams compared to inverted trigrams (Figure S13C). Finally we confirmed that model terms for upright and inverted trigrams were highly correlated (correlation between upright and inverted model terms, averaged across 9 model term types: $r=0.65 \pm 0.1, p<0.05$ in all cases).

We conclude that upright and inverted trigram searches can be explained using the part-sum model driven by a common single letter representation.

A

B
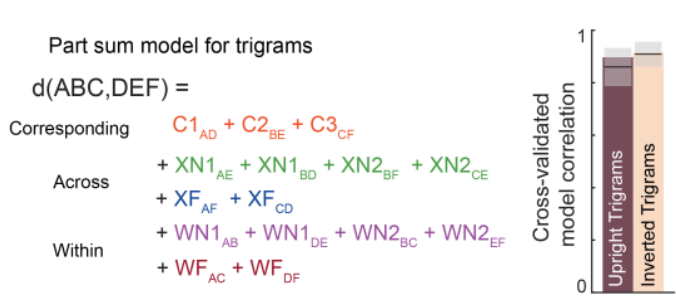

C

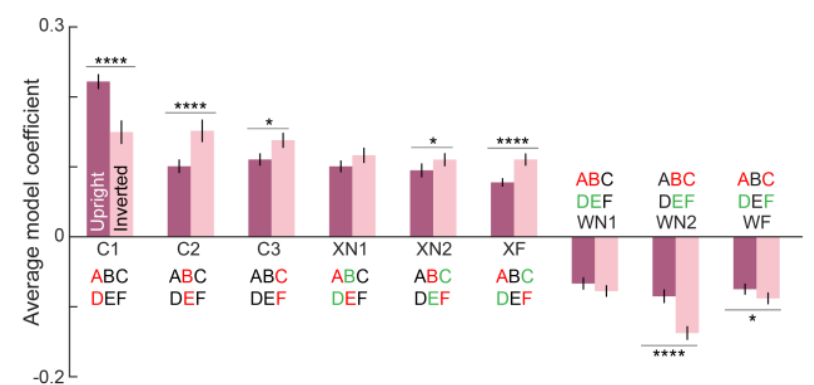

Figure S13. Part-sum model fits for upright and inverted trigrams

(A) Schematic of part-sum model for trigrams.

(B) Cross-validated model correlation of part-sum model for upright and inverted trigrams.

(C) Average model coefficient (averaged across ${ }^{6} \mathrm{C}_{2}=15$ terms) of each type for upright and inverted trigrams. Asterisks indicate statistical significance $\left(^{*}\right.$ is $p<$ $0.05,{ }^{* *}$ is $p<0.005$, etc) calculated using a sign-rank test comparing the upright and inverted model terms. 
Here, in Experiment S6, we tested subjects on a jumbled word reading task,

METHODS

Procedure. A total of 16 subjects (9 male, aged $24.8 \pm 2.1$ years) participated in the task. Other details were similar to Experiment 5.

858 Stimuli. We chose 300 words such that no two words were anagrams of each other. These comprised 75 four-letter words, 150 five-letter words and 75 six-letter words. Jumbled words were created by shuffling 2, 3, or 4 letters of each word. There were an equal proportion of 2, 3, and 4 letter transpositions. All stimuli were presented in uppercase against a black background.

863 Task. Each trial began with a fixation cross shown for $0.5 \mathrm{~s}$ followed by a jumbled word that appeared for 5 seconds (for the first 6 subjects) and 7 seconds (for the rest), or until the subject made a response by pressing the space bar on the keyboard. Subjects were asked to press a key as soon as they could recognize the unjumbled word. To ensure that subjects correctly recognized the unjumbled word, they were asked to type the unjumbled word within 10 seconds of pressing the space bar. The response time was taken as the time at which the subject pressed the space bar. To avoid any memory effects, the same set of jumbled words were shown to all subjects exactly once. We analysed response times only on trials in which the subject subsequently entered the correct word.

Data Analysis. Subjects were reasonably accurate on this task (average accuracy: $59.5 \pm 8 \%$ across 300 words). Response times for wrongly typed words were discarded. Words correctly solved by more than 6 subjects $(n=238)$ were included for further analysis. Since trials were self-paced, we did not remove any outliers in the reaction times. Lexical properties were obtained from the English Lexicon Project (Balota et al., 2007).

\section{RESULTS}

Of a total of 300 jumbled words tested, we selected for further analysis 238 words that were correctly unjumbled by more than two-thirds of the subjects. Subjects responded quickly and accurately to these words (mean \pm std of accuracy: $71 \pm 9 \%$; response time: $2.13 \pm 0.33 \mathrm{~s}$ across 238 words). Subjects took longer to respond to some jumbled words (e.g. REHID) compared to others (e.g. DBTOU), as seen in the sorted response times (Figure S14A). These patterns were consistent across subjects, as evidenced by a significant split-half correlation $(r=0.55, p<0.00005$ between oddand even-numbered subjects).

Can these patterns in unscrambling time be explained using the letter model? To do so, we reasoned that jumbled words with large dissimilarity to the original word will take longer to elicit a response (Figure S14B). Accordingly, we took the average response times to each jumbled word and asked whether it can be predicted using the 
weights of the single letter model to find the best fit to this data, and then combined the predictions across all word lengths to obtain a composite measure of performance. The single letter model yielded excellent fits to the data $(r=0.76, p<0.00005$; Figure $\mathrm{S} 14 \mathrm{C})$. This model fit was comparable to the data consistency ( $\left.r_{\text {data }}=0.70\right)$. An alternate distance model - Orthographic Levenshtein (OL) distance (Levenshtein, 1966) - calculates the number of edits required to transform one string to other. This model neither accounts for letter similarity nor the position of edit. Hence, it fails to account for all the variance in the data $(r=0.44, p<0.00005$; Figure S14D).

The above finding shows that human performance on unscrambling words is driven primarily by the visual dissimilarity between the jumbled and original word. However, it does not rule out the presence of lexical factors. To assess this possibility we formulated a model to predict the unscrambling time as a linear sum of many lexical factors. We used five lexical properties: log word frequency, log mean letter frequency, log mean bigram frequency of the jumbled word, log mean bigram frequency of the unjumbled i.e. original word, and the number of orthographic neighbours (see Methods). To avoid overfitting by either model, we trained both models on one-half of the subjects and tested it on the other half. This lexical model yielded relatively poor fits $(r=0.30, p<0.00005$, Figure $S 14 E)$ compared to visual dissimilarity from both single letter model and OL distance model. The difference in model fits was statistically significant ( $p<0.05$, Fisher's $z$-test). Among the lexical factors, word frequency and letter frequency contributed the most compared to the others (partial correlation of each lexical factor after accounting for all others: $r=-0.23, p<0.0005$ for log word frequency, $r=0.18, p<0.05$ for log mean letter frequency; $r=.05, p=0.49$ for $\log$ mean bigram frequency of jumbled word; $r=-0.02, p=0.77$ for log mean bigram frequency in original word; $r=0.04, p=0.58$ for number of orthographic neighbours).

To assess the extent of shared variance in the two models, we calculated the partial correlation between the observed data and the lexical model predictions after factoring out the contribution from visual dissimilarity. This revealed a small partial correlation $(r=0.31, p<0.00005)$. Conversely, the partial correlation for the single letter model after factoring out the lexical model was much higher $(r=0.75, p<$ $0.00005)$. Thus, visual dissimilarity from the single letter model dominates jumbled word reading.

Finally we asked whether both visual dissimilarity and lexical factors contribute to the jumbled word task. We created a combined model in which the jumbled word response times were a linear combination of the predictions of both models. This combined model yielded better predictions than either model by itself $(r=0.78, p<$ 0.00005 , Figure S14E). To assess the statistical significance of these results, we performed a bootstrap analysis. On each trial, we trained three models on the dissimilarity obtained from considering only one randomly chosen half of subjects: the visual dissimilarity model, the lexical model and the combined model. We calculated the correlation between all three model predictions on the other half of the data, and repeated this procedure 1000 times. The OL distance model does not have any free parameters, hence the distances were directly correlated with the other half of the data. Across these samples, the lexical model fits never exceeded the visual 
938 dissimilarity model, suggesting that the visual dissimilarity model was significantly

939 better $(p<0.05)$. Likewise, the combined model was only marginally better than the

940 visual letter model (fraction of combined $<$ visual: $p=0.07$ ) but was significantly better

941 than the lexical model (fraction of combined < lexical: $p=0$ ).

942 We conclude that performance on the jumbled word task relies primarily on

943 visual dissimilarity. We propose that this initial visual representation of a word allows

944 the subject to make a quick guess at the correct word without explicit symbolic 945 manipulation.

946

947 


\section{Scrambled word task}

A

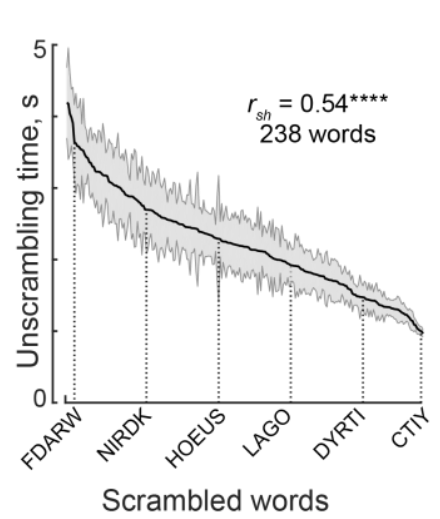

B

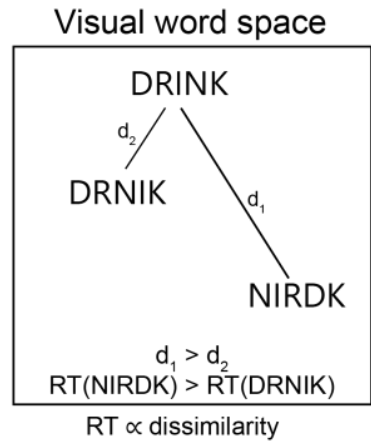

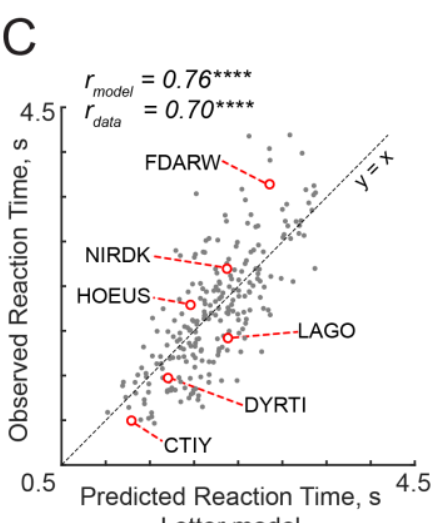

Letter model

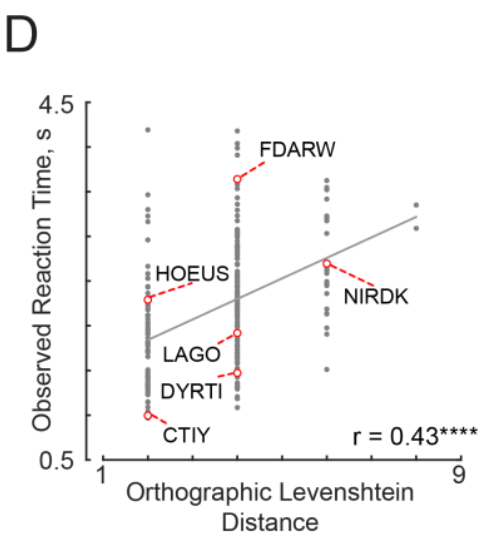

E

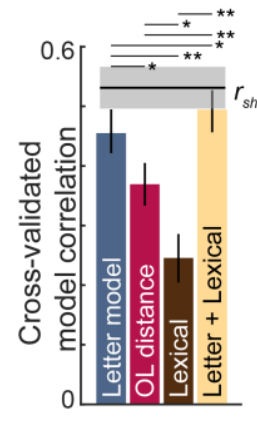

Figure S14. Jumbled word task (Experiment S7).

(A) Response times in the jumbled word task sorted in descending order. Shaded error bars represent s.e.m. Some example words are indicated using dotted lines. The split-half correlation between subjects $\left(r_{s h}\right)$ is indicated on the top left.

(B) Schematic of visual word space, with one stored word (DRINK) and two jumbled versions (DRNIK \& NIRDK). We predicted that the time taken by subjects to unscramble a jumbled word would be proportional to its dissimilarity to the stored word. Thus, subjects would take longer to unscramble NIRDK compared to DRINK.

(C) Observed response times in the jumbled word task plotted against predictions from the letter model based on single letters with spatial summation. Each point represents one word. Asterisks indicate statistical significance $\left({ }^{* * *}\right.$ is $\left.p<0.00005\right)$.

(D) Observed response times in the jumbled word task plotted against Orthographic Levenshtein (OL) distance. Each point represents one word. Asterisks indicate statistical significance $\left({ }^{* * * *}\right.$ is $\left.p<0.00005\right)$.

(E) Cross-validated model correlations for the letter model, OLD model, lexical model and the neural+lexical model. Model correlations were obtained by training each model on one half of subjects, and evaluating the correlation on the other half (error bars represent standard deviation across 1000 random splits). The upper bound on model fits is the split-half correlation $\left(r_{s h}\right)$, shown in black with shaded error bars representing standard deviation across the same random splits. All correlations were individually statistically significant $(p<0.00005)$. Horizontal lines above shaded error bar depicts significant difference across different models i.e. the fraction of splits in which the observed difference was violated. All significant comparisons are indicated. 
975

976

977

978

979

980

981

982

983

984

985

986

\section{Stimulus set}

32 words were chosen of varying frequency of occurrence and the nonwords were created by either transposition or substitution of middle or edge letters. 10 single letters: $E, S, A, R, O, L, I, T, N$, and D were used to form words. The full set of strings used experiments 6 and 7 is shown below.

\begin{tabular}{|c|c|c|c|c|c|c|c|}
\hline \multicolumn{2}{|c|}{$\begin{array}{c}\text { Middle Letter } \\
\text { Transposition }\end{array}$} & \multicolumn{2}{c|}{$\begin{array}{c}\text { Edge Letter } \\
\text { Transposition }\end{array}$} & \multicolumn{2}{c|}{$\begin{array}{c}\text { Middle Letter } \\
\text { Substitution }\end{array}$} & \multicolumn{2}{c|}{$\begin{array}{c}\text { Edge Letter } \\
\text { Substitution }\end{array}$} \\
\hline Words & Nonwords & Words & Nonwords & Words & Nonwords & Words & Nonwords \\
\hline AORTA & AROTA & STOLE & TSOLE & NOISE & NANSE & ONION & ESION \\
DRAIN & DARIN & OASIS & AOSIS & ERROR & EDLOR & RADIO & EEDIO \\
TREND & TERND & SOLID & OSLID & DRILL & DTELL & ASSET & EESET \\
ATLAS & ALTAS & TRAIN & RTAIN & ARISE & AOESE & TEASE & RDASE \\
DRONE & DRNOE & ORDER & ORDRE & LITRE & LINOE & ENTER & ENTRO \\
LEARN & LERAN & INDIA & INDAI & SLIDE & SLONE & IDEAL & IDEDI \\
SANTA & SATNA & RINSE & RINES & NASAL & NATDL & ADORE & ADODI \\
INSET & INEST & SNAIL & SNALI & ALIEN & ALOTN & LASER & LASRO \\
\hline
\end{tabular}

Table S3: List of 32 words and 32 nonwords used in Experiment 6 \& 7 . All words and nonwords were created from 10 single letters whose activations were also measured in the experiment.

\section{ROI definitions}

\begin{tabular}{|c|c|c|c|}
\hline ROI & Definition & $\begin{array}{c}\text { \#voxels } \\
\text { (mean } \pm \text { sd) }\end{array}$ & $\begin{array}{l}\text { ROI peak } \\
\text { location }\end{array}$ \\
\hline V1-V3 & $\begin{array}{l}\text { Voxels activated for scrambled }>\text { fixation } \\
\text { overlaid with anatomical mask of } \mathrm{V} 1-\mathrm{V} 3\end{array}$ & $398 \pm 131$ & $\begin{array}{l}X: 8 \pm 17 \\
Y:-96 \pm 5 \\
Z: 6 \pm 9\end{array}$ \\
\hline V4 & $\begin{array}{l}\text { Voxels activated for scrambled > fixation } \\
\text { overlaid with anatomical mask of V4 }\end{array}$ & $185 \pm 63$ & $\begin{array}{l}X: 5 \pm 26 \\
Y:-88 \pm 3 \\
Z: 27 \pm 11\end{array}$ \\
\hline LO & $\begin{array}{l}\text { Voxels activated for object > scrambled and } \\
\text { not in other ROls }\end{array}$ & $371 \pm 115$ & $\begin{array}{l}X:-17 \pm 43 \\
Y:-66 \pm 15 \\
Z:-19 \pm 5\end{array}$ \\
\hline VWFA & $\begin{array}{l}\text { Voxels with known words > scrambled word } \\
\text { in a contiguous region in fusiform gyrus }\end{array}$ & $52 \pm 15$ & $\begin{array}{l}X:-44 \pm 4 \\
Y:-50 \pm 5 \\
Z:-17 \pm 5\end{array}$ \\
\hline TG & $\begin{array}{l}\text { Voxels with native words > scrambled word in } \\
\text { a contiguous region in temporal gyrus }\end{array}$ & $289 \pm 182$ & $\begin{array}{l}X:-44 \pm 39 \\
Y:-43 \pm 18 \\
Z: 3 \pm 9\end{array}$ \\
\hline
\end{tabular}

987

988

989

990

991

992

993
Table S4. Variability in ROI definitions across subjects. For each ROI we report the mean and standard deviation across subjects of the number of voxels, and the $\mathrm{XYZ}$ location of the voxel with peak T-value in the normalized brain. 


\section{Visualization of perceptual and semantic space}

To visualize words and nonwords in perceptual space, we performed a multidimensional scaling (MDS) analysis of the visual search data (Experiment 7).

997 Briefly, MDS finds the best-fitting 2D coordinates that best match with the observed 998 distances. In the resulting plot, nearby stimuli correspond to hard searches. The perceptual space for words and nonwords is shown in Figure S15 A-B. It can be seen that stimuli with common first letters are grouped together. MDS coordinates for nonwords was rotated without altering their overall configuration so as to best match the MDS coordinates for words.

The semantic dissimilarities were estimated using the GloVe features

A

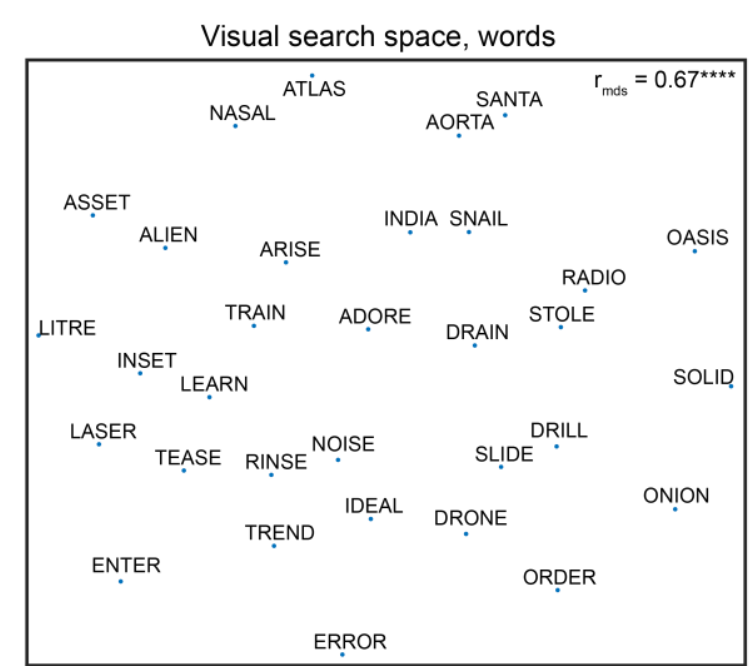

B

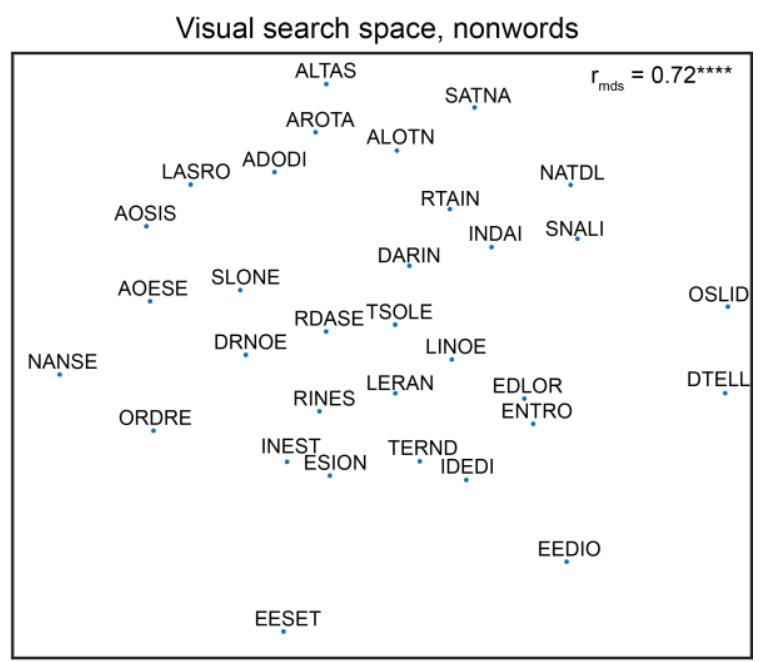
(Pennington et al., 2014), and visualized using MDS analysis (Figure S15C). In the resulting plot, semantically related words/ frequently cooccurring words are closer to each other.

\section{C}

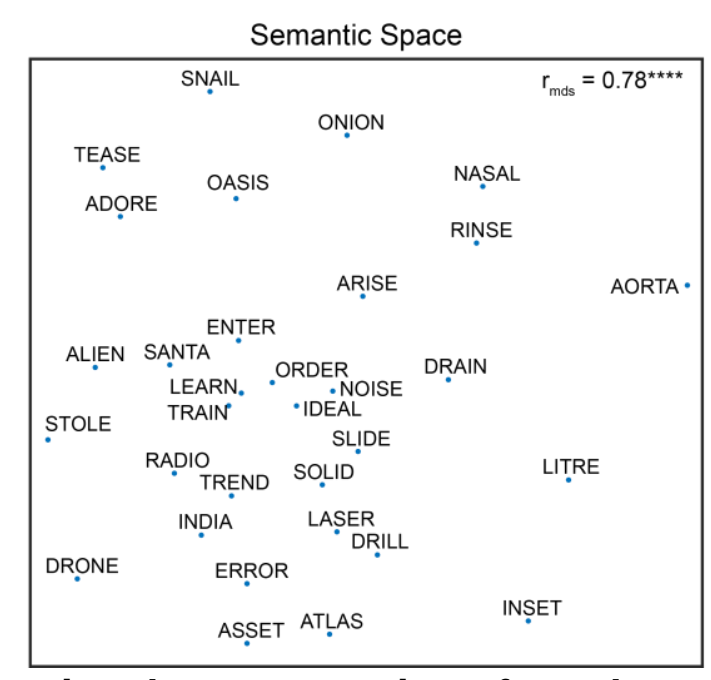

Figure S15: Multi-dimensional representation of words and nonwords.

A. Perceptual space for words. we used multidimensional scaling to find the $2 \mathrm{D}$ coordinates of all words that best match the observed distances. In the resulting plot, nearby words indicate hard searches. The correlation coefficient between dissimilarities in 2D plane and the observed data is shown. Asterisks indicate significant correlation $\left({ }^{* * * *}\right.$ is $\left.p<0.00005\right)$.

B. Same as (A) but for nonwords. 
1016

1017

1018

1019

1020

1021

1022

1023

1024

1025

1026

1027

1028

1029

1030

1031

1032

1033

1034

1035

1036

1037

1038

1039

1040

1041

1042

1043

1044

1045

1046

1047

1048

1049

1050

1051

1052

1053

1054

1055

1056

1057

1058

1059

1060

1061

1062

1063

C. Same as (A) but for semantic space of words.

\section{Neural activity corresponding to words, nonwords, and letters}

For each category of stimuli i.e. words, nonwords, and letters, we averaged the activity values across voxels and subjects within each $\mathrm{ROI}$. The mean activity values are shown in Figure S16A-E.

\section{Word vs nonword classification}

For each ROI and subject, we built linear classifier to discriminate between words and nonwords using built-in MATLAB routine "fitcdiscr". We built separate classifiers to distinguish the activity pattern of transposed and substituted nonwords from their corresponding word activity patterns. The resulting decoding accuracy is shown in Figure S16F.

\section{Can string responses be predicted from single letters?}

We modelled the response of each voxel across the 64 strings (32 words, 32 nonwords) as a linear combination of the single letter activations (Figure S16G). We evaluated model fits by comparing model correlations separately for words and nonwords. If string responses were driven by specialized detectors for letter combinations (such as those present in words), then we reasoned that model correlations would be worse for words compared to nonwords. By contrast, if there are no specialized detectors of this kind, model fits would be equivalent for words and nonwords.

We calculated cross-validated model fits by training the model on half the trials and testing it on the other half of the trials. Since voxels could vary widely in their reliability of responses to the stimuli, we normalized the model fit of each voxel by its split-half reliability. The average noise-corrected model fit (averaged across voxels and subjects) is shown in Figure $\mathrm{S} 16 \mathrm{H}$. This revealed no systematic difference in model performance for words and nonwords in any of the ROls (Figure $\mathrm{S} 16 \mathrm{H}$ ). We obtained qualitatively similar results using a searchlight, where there were no clear regions in which model fits differed for words and nonwords (Figure S17D).

To further validate the letter model, we compared the single letter tuning along each MDS dimension with the observed single letter tuning in each ROI (Figure S18A). For each ROI, we grouped voxels with similar response profile and matched it to the MDS dimension (Figure S18A). We obtained similar single letter tuning and weight profiles for voxels across different ROls. However this analysis is inconclusive because there is no systematic way to compare a small set of neurons inferred from behaviour with the much larger, possibly overcomplete set of voxel activations observed in brain imaging. Likewise, we grouped voxels with similar summation weights to compare the weight profiles in behaviour and brain imaging. However this analysis is also inconclusive because different MDS-derived neurons might contribute differently towards behaviour, so the summation weights cannot be directly averaged to make overall comparisons between ROI activations and behaviour. Despite these caveats, there is a general match between tuning profiles and summation weights observed in behaviour with those observed in different brain regions. 
A

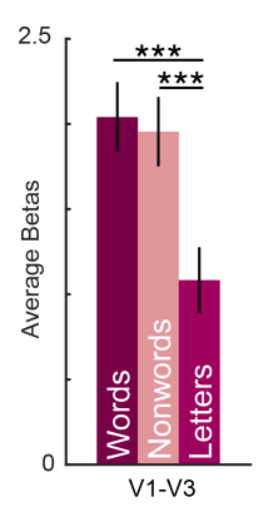

B

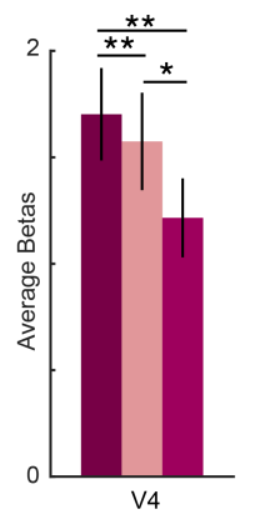

C

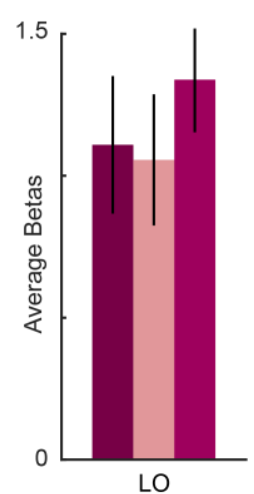

D

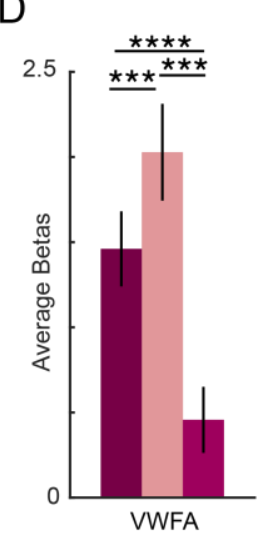

$E$

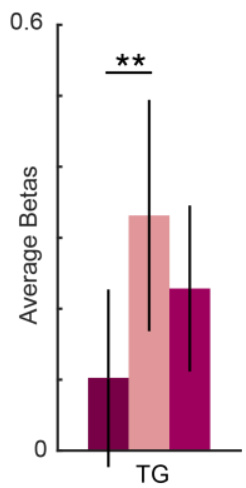

$\mathrm{F}$

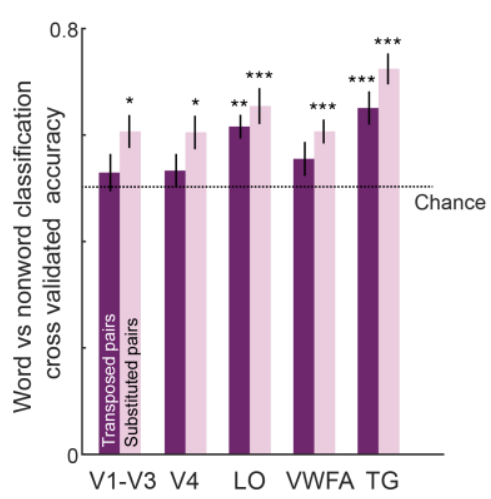

G Modeling string activations using letter activations
1064

\section{Figure S16: Neural activity}

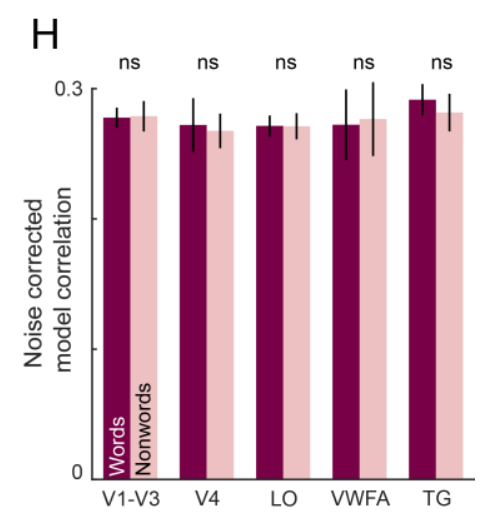
(A) Average activation levels for words, nonwords, and letters. Error bar indicate \pm 1 s.e.m. across subjects. Asterisks indicate statistical significance ( ${ }^{*}$ is $p<$ $0.05,{ }^{* *}$ is $p<0.005$, etc. in a sign-rank test comparing subject-wise average activations).

(B)-(E). Same as in A but for V4, Lateral Occipital areas, Visual Word Form Area, and Temporal Gyri respectively.

(F) Cross-validated classification accuracy for transposed word-nonword pairs (dark) and substituted word-nonword pairs (light). Error bars indicate s.e.m. across subjects. Asterisks indicate statistical significance $\left({ }^{*}\right.$ is $p<0.05$, ${ }^{* *}$ is $p$ $<0.005$, etc. in a sign-rank test comparing subject-wise accuracy w.r.t. chance level).

(G) Schematic of the voxel model. The response of each voxel across strings is modelled as a linear combination of the constituent letter responses. Bottom: Hypothetical model fits based on the presence (right) or absence (left) of local combination detectors. Predicted responses for words will deviate from the observed responses under the influence of LCD.

(H) Average model correlation (normalized using split-half correlation) for each ROI for words (dark) and nonwords (light). Error bar indicate s.e.m. across subject. 


\section{Searchlight analyses}

To identify other brain regions that might show the effects observed in the individual ROls, we performed a whole-brain searchlight analysis. Specifically, for each voxel in a given subjects' brain, we considered a local neighbourhood of 27 voxels ( $3 \times 3 \times 3$ voxels) and performed the following analyses of interest. We obtained similar results for larger searchlight volumes. The resulting maps were smoothed using a Gaussian filter with FWHM of $3 \mathrm{~mm}$

\section{Searchlight for regions that match lexical decision time}

For each voxel, its activity across strings is correlated with mean lexical decision time. The resulting whole brain correlation map is averaged across subjects. Overall, activity in VWFA, Superior Parietal Lobe (SPL), Pre-Frontal and motor cortex is correlated with lexical decision time. This correlation map was visualized on the brain surface (Figure S17A).

\section{Searchlight for regions that match perceptual space}

For the neighbourhood of each voxel, we calculated the pairwise neural dissimilarity for all word-word, nonword-nonword, and word-nonword pairs for a given subject, and averaged this across subjects. We then calculated the correlation between this local neural dissimilarity and the corresponding string dissimilarities estimated using experiment 7. This correlation map was visualized on the brain surface (Figure S17B).

\section{Searchlight for regions that match semantic space}

For the neighbourhood of each voxel, we calculated the pairwise neural dissimilarity for all word-word pairs for a given subject and averaged this across subjects. We then calculated the correlation between this local neural dissimilarity and the corresponding semantic dissimilarities. This correlation map was visualized on the brain surface (Figure S17C).

\section{Searchlight for comparing linear model fits between words and nonwords}

For each subject and voxel, we modelled the response to strings as a linear combination of its single letter responses. The model fits (correlation between observed and predicted string responses) was evaluated separately for words and nonwords. The difference in the mean model fits between words and nonword is visualized on the brain surface (Figure S17D). 
A

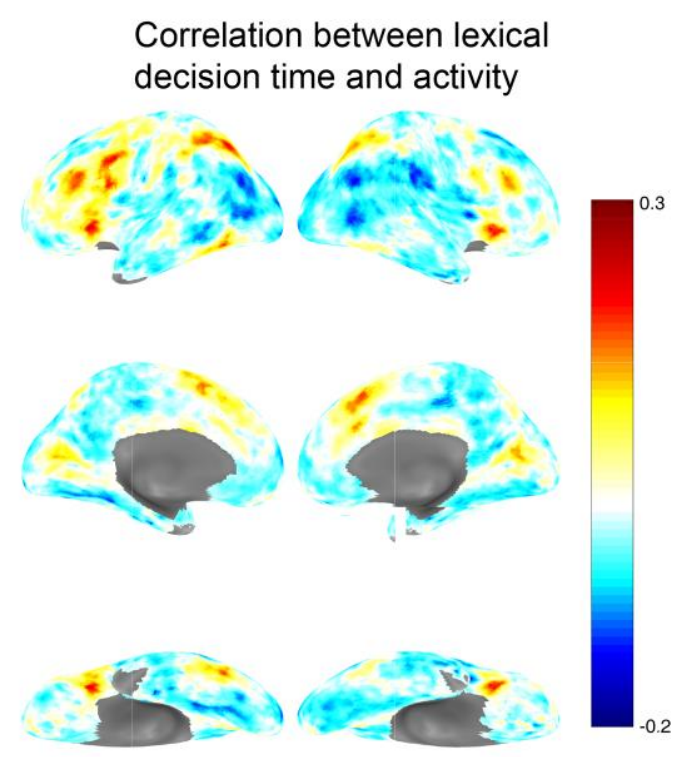

C

Match to semantic space
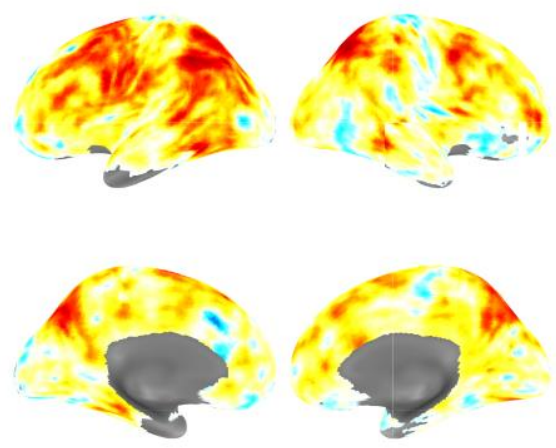

1126

1127

1128

1129

1130

1131

1132

1133

1134

1135

1136

\section{Figure S17: Searchlight analysis} each voxel. dissimilarities in behaviour. dissimilarities. for each voxel, averaged across subjects.
B Match to perceptual space
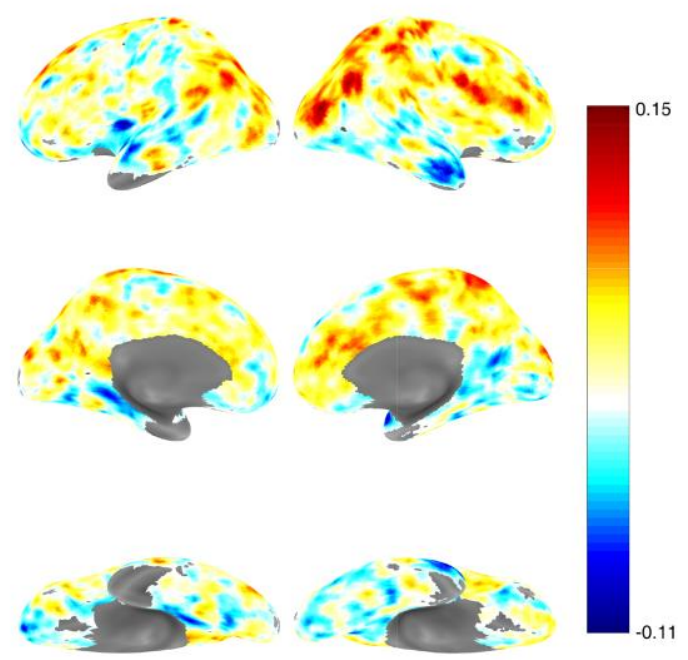

D

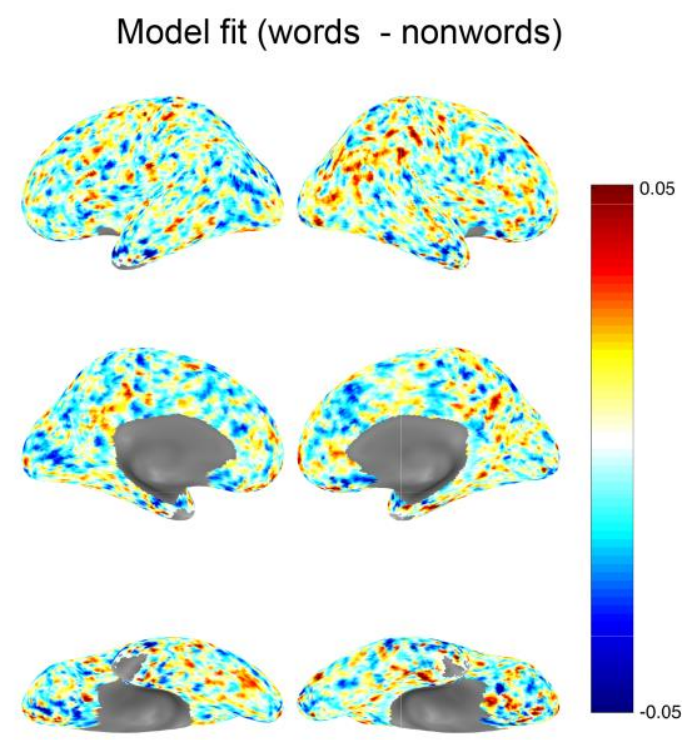

A. Searchlight map of correlation between neural activity and lexical decision time for

B. Searchlight map of correlation between neural dissimilarity and search

C. Searchlight map of correlation between neural dissimilarity and semantic

D. Searchlight map depicting the difference in model fit for words versus nonwords 


\section{Match between letter model and fMRI data}

The letter model described throughout the study is derived from dissimilarities measured in behaviour in two steps. First, the dissimilarities between single letters were used to construct single neurons tuned to letter shape, whose activity predicts these dissimilarities. Second, the summation weights of each neuron were adjusted so that they match the dissimilarities between longer strings.

Given that we recorded responses to single letters as well as strings in $\mathrm{fMRI}$, we wondered whether these can be matched in some manner to the letter tuning and summation weights derived from behaviour in the letter model. Any direct comparison is fraught with the difficulty that many single letter tuning functions could produce the same behaviour. For instance, simply rotating the MDS-derived tuning functions could yield another set of neurons that match the observed letter dissimilarities. This is further compounded by the fact that the MDS-derived neurons contribute unequally to behaviour, and by the fact that this mapping could change completely with increasing numbers of neurons. Thus it is unreasonable to expect voxel tuning for single letters or the summation weights to match exactly with the behaviourally derived tuning.

Nonetheless, we attempted to find a broad link between the single letter tuning and summation weights observed in behaviour with those observed in each ROI. The results are summarized in Figure S18. Since there are only 10 single letters, 6 MDS neurons were sufficient to explain $>95 \%$ of the variance of the pair-wise single letter dissimilarities observed in Experiment 1. For each MDS neuron, we identified the voxels whose activity for single letters had the least residual error compared to other MDS neurons. In this manner, we sorted the voxels into six groups corresponding to each MDS neuron. The resulting plots are shown in Figure S18A. It can be see that all ROls show single letter tuning profiles similar to the behaviourally derived single letter tuning profiles. The corresponding summation weights for these voxels are shown in Figure S18B. Once again, it can be seen that many ROls show similar summation weights as those observed in behaviour. 
1165

A Letter tuning
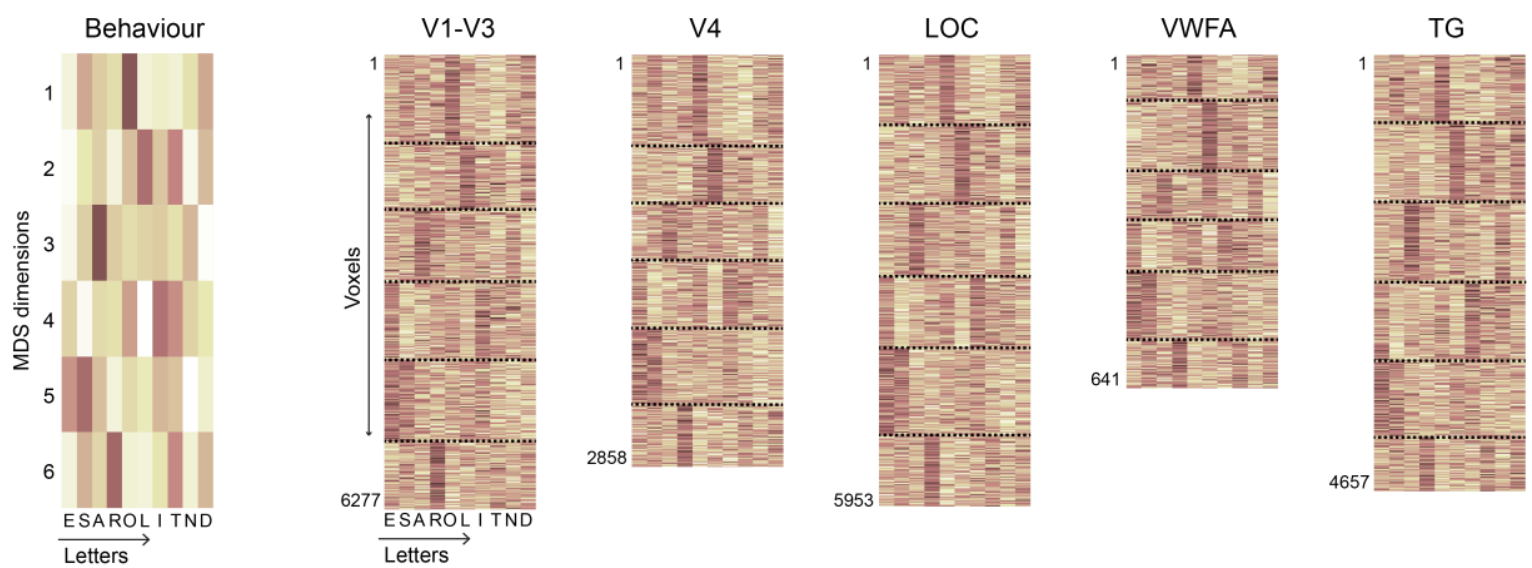

B Summation weights
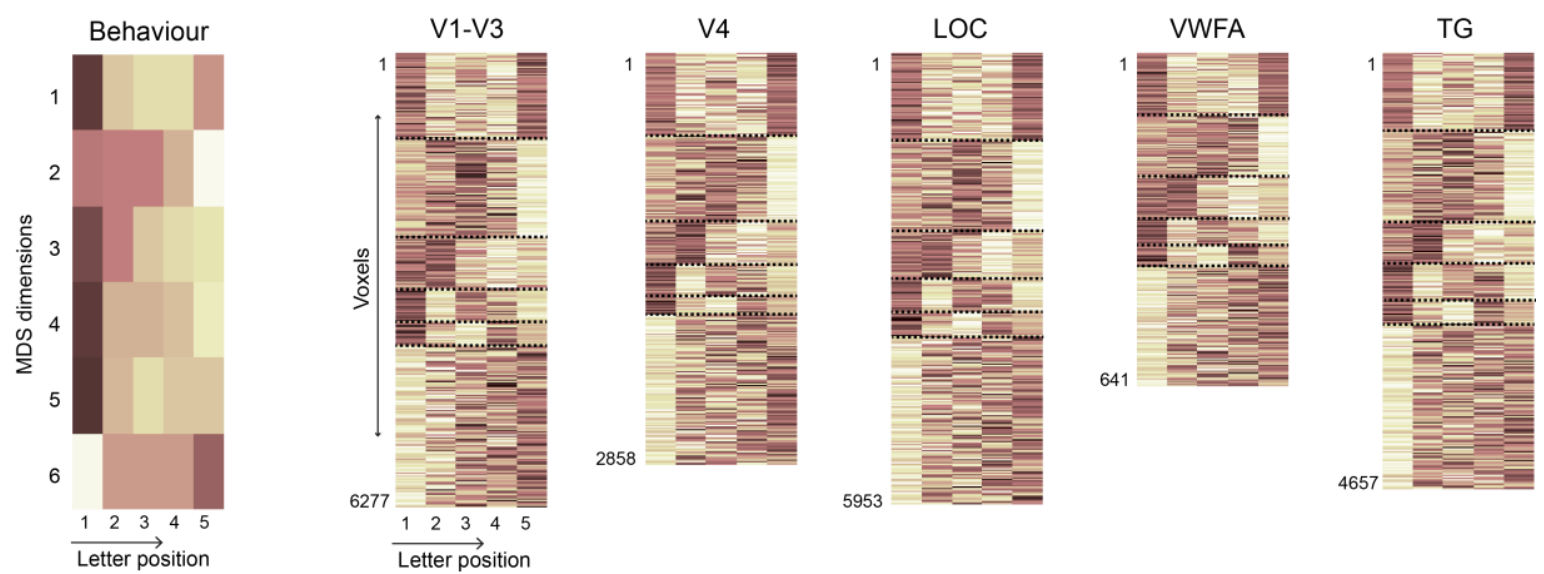

1166

Figure S18: Comparison of letter tuning and summation weights

1168

A. (Left) Response of 6 MDS neurons for all the 10 letters. (Right) Single letters response across all the voxels (concatenated across subjects) within a given ROI. Each voxels is sorted into one of 6 groups depending on which MDS neuron it matches best. The height of each ROI plot is logarithmically scaled to match the number of voxels across all subjects. Black dashed lines are used to separate the clusters corresponding to each MDS neuron.

1174

B. Same as (A) but showing the summation weights corresponding to each MDS neuron or ROI voxel. 
Balota DA et al. (2007) The English Lexicon Project. Behav Res Methods 39:445-459.

Dehaene S, Cohen L, Sigman M, Vinckier F (2005) The neural code for written words: a proposal. Trends Cogn Sci 9:335-341.

Duncan J, Humphreys GW (1989) Visual search and stimulus similarity. Psychol Rev 96:433-458.

Levenshtein V (1966) Binary codes capable of correcting deletions, insertions, and reversals. Sov Phys Dokl 10:707-710.

Mueller ST, Weidemann CT (2012) Alphabetic letter identification: Effects of perceivability, similarity, and bias. Acta Psychol (Amst) 139:19-37.

Pennington J, Socher R, Manning CD (2014) GloVe: Global Vectors for Word Representation. In: Empirical Methods in Natural Language Processing (EMNLP), pp 1532-1543.

Pramod RT, Arun SP (2016) Object attributes combine additively in visual search. J Vis $16: 8$.

Ratan Murty NA, Arun SP (2015) Dynamics of 3D view invariance in monkey inferotemporal cortex. J Neurophysiol 113:2180-2194.

Simpson IC, Mousikou P, Montoya JM, Defior S (2013) A letter visual-similarity matrix for Latin-based alphabets. Behav Res Methods 45:431-439.

Vighneshvel T, Arun SP (2013) Does linear separability really matter? Complex visual search is explained by simple search. $\mathrm{J}$ Vis 13:1-24. 